\title{
Radiation-reaction force and multipolar waveforms for eccentric, spin-aligned binaries in the effective-one-body formalism
}

\author{
Mohammed Khalil@, ${ }^{1,2, *}$ Alessandra Buonanno, ${ }^{1,2, \dagger}$ Jan Steinhoff $\odot,{ }^{1, \$}$ and Justin Vines $\oplus^{1, \S}$ \\ ${ }^{1}$ Max Planck Institute for Gravitational Physics (Albert Einstein Institute), \\ Am Mühlenberg 1, Potsdam 14476, Germany \\ ${ }^{2}$ Department of Physics, University of Maryland, College Park, Maryland 20742, USA
}

(Received 4 May 2021; accepted 16 June 2021; published 16 July 2021)

\begin{abstract}
While most binary inspirals are expected to have circularized before they enter the LIGO/Virgo frequency band, a small fraction of those binaries could have non-negligible orbital eccentricity depending on their formation channel. Hence, it is important to accurately model eccentricity effects in waveform models used to detect those binaries, infer their properties, and shed light on their astrophysical environment. We develop a multipolar effective-one-body (EOB) eccentric waveform model for compact binaries whose components have spins aligned or antialigned with the orbital angular momentum. The waveform model contains eccentricity effects in the radiation-reaction force and gravitational modes through second post-Newtonian (PN) order, including tail effects, and spin-orbit and spin-spin couplings. We recast the $\mathrm{PN}$-expanded, eccentric radiation-reaction force and modes in factorized form so that the newly derived terms can be directly included in the state-of-the-art, quasi-circular-orbit EOB model currently used in LIGO/Virgo analyses (i.e., the SEOBNRV4HM model).
\end{abstract}

DOI: $10.1103 /$ PhysRevD.104.024046

\section{INTRODUCTION}

The observation of gravitational waves (GWs) by the LIGO-Virgo detectors [1,2] have corroborated the existence of binary black holes (BBHs) in our universe. But how and in which astrophysical environments these binaries form are not yet fully understood. However, the masses, spins (magnitude and orientation), and binary eccentricities inferred from GWs provide invaluable clues to determine BBH formation channels $[3,4]$. So far, the observed GWs are consistent with binary coalescences of negligible eccentricity, i.e., on quasicircular orbits [5-8].

In general, binaries are expected to circularize $[9,10]$ as they approach merger due to the emission of gravitational radiation. But depending on their astrophysical formation channel, a small fraction of binaries could have nonnegligible orbital eccentricity, as they enter the frequency bands of current detectors. This can occur in dense stellar environments, such as globular clusters or galactic nuclei,

*mohammed.khalil@aei.mpg.de

†alessandra.buonanno@aei.mpg.de

*jan.steinhoff@aei.mpg.de

§ustin.vines@aei.mpg.de

Published by the American Physical Society under the terms of the Creative Commons Attribution 4.0 International license. Further distribution of this work must maintain attribution to the author(s) and the published article's title, journal citation, and DOI. Open access publication funded by the Max Planck Society. where dynamic capture [11-16] or the Lidov-Kozai mechanism in hierarchical triples [17-19] can lead to eccentric binary inspirals at close separations.

In particular, Ref. [13] (and Ref. [14]) showed that 5\% (or $\sim 10 \%$ ) of all mergers in globular clusters enter the LIGO band with eccentricity $e>0.1$. Binaries formed via dynamic capture in galactic nuclei are expected to have high eccentricities [16], with $92 \%$ having $e>0.1$ and $50 \%-85 \%$ having $e>0.8$ at $10 \mathrm{~Hz}$. For a $\mathrm{BBH}$ around a supermassive $\mathrm{BH}$, the Lidov-Kozai mechanism can secularly drive the $\mathrm{BBH}$ to eccentricities near unity for some orientations [19]. Hence, inferring those eccentricities from GWs is important for understanding the origin and environment of BBHs. Interestingly, Ref. [8] pointed out that GW190521 [20] could be consistent with either an eccentric nonprecessing or a quasicircular precessing binary, which illustrates both the difficulties and the prospects of further observations in the upcoming and future LIGO, Virgo, and KAGRA runs [21].

While the expected fraction of eccentric GW observations with current detectors is small, neglecting eccentricity for the parameter inference can cause significant bias [22]. This becomes more relevant for LISA where a large fraction of stellar-mass binaries is expected to be eccentric [23-27]. Hence, it is important to develop accurate waveform models for eccentric binaries to detect them, infer their properties, and shed light on their astrophysical environment and formation channels. Several studies developed post-Newtonian (PN) waveform models for eccentric 
orbits, such as Refs. [28-38], or hybrid models that use PN results for the inspiral and quasicircular numericalrelativity (NR) simulations near merger [39-41]. Recently, NR simulations for eccentric binaries were reported in Refs. [41-43], and the first NR surrogate model for eccentric BBHs has been developed in Ref. [44].

The effective-one-body (EOB) formalism [45-47] improves inspiral-merger-ringdown waveforms by combining information from PN theory, NR simulations, and the strong-field test-body limit. EOB Hamiltonians have been constructed to include spin [48-56], tidal effects [57-60], information from the small mass-ratio [61-65] and the post-Minkowskian approximations [66-68], and have been refined and calibrated to NR simulations [69-77]. While the EOB Hamiltonian is valid for generic orbits, most EOB waveform models use quasicircular orbit results for the radiation-reaction (RR) force, gravitational waveform modes, and the calibration with NR simulations.

Recent approaches to extend the EOB formalism to eccentric orbits include Ref. [78], which derived the RR force with eccentricity up to $2 \mathrm{PN}$ order, but without tail effects and for nonspinning BHs. More recently, Ref. [79] incorporated eccentricity effects in the RR force and in the $(2,2)$ waveform mode through $1.5 \mathrm{PN}$ order, including tail effects, using the Keplerian parametrization and phase variables that evolve only due to $\mathrm{RR}$. References $[80,81]$ extended the quasicircular SEOBNRv1 [72] model to eccentric orbits, while Ref. [82] added eccentric corrections in the SEOBNRV4 $[74,83]$ waveform model, notably in the $(2,2),(2,1),(3,3),(4,4)$ modes through $2 \mathrm{PN}$ order, including spin-orbit (SO) and spin-spin (SS) couplings, ${ }^{1}$ but not tail effects. They employed these eccentric modes to construct a RR force for eccentric orbits, which, however, does not include the Schott terms. As argued in Ref. [78] and Sec. II below, these Schott terms are necessary for generic orbits to satisfy the flux-balance equations. Furthermore, Refs. [84,85] incorporated noncircular effects in the TEOBResums_SM $[76,86]$ model at leading PN order in the azimuthal component of the RR force and used a quasicircular 2PN-expanded radial RR force without spin or tail effects. They included eccentric corrections at leading PN order to all modes $m \neq 0$ up to $\ell=|m|=5$.

In this paper, we develop a multipolar EOB waveform model for eccentric binaries with the compact-objects' spins aligned or antialigned (henceforth, for short aligned) with the orbital angular momentum. We derive the eccentric PN expressions for the RR force (including the Schott terms) and the gravitational modes up to $\ell=|m|=6$, including the $m=0$ mode, through 2PN order, including tail effects, and SO and SS couplings. We recast our results for the RR force and modes in a form that can be directly

\footnotetext{
${ }^{1}$ Our results for those modes are mostly in agreement with Ref. [82] except for the SO part, where we disagree with their findings (their expressions contain two extra SO terms).
}

incorporated in the state-of-the-art, quasi-circular-orbit EOB model currently used in LIGO/Virgo analyses (SEOBNRV4HM $[74,83]$ ).

The paper is structured as follows. In Sec. II, we derive the $\mathrm{RR}$ force from the energy and angular momentum fluxes using the balance relations. We use the gauge freedom in the $\mathrm{RR}$ force to impose that it reduces to the relation used in SEOBNRV4HM in the quasi-circular-orbit limit. In Sec. III, we obtain initial conditions for eccentric orbits. In Sec. IV, we calculate all the gravitational waveform modes that contribute up to $2 \mathrm{PN}$ order relative to the leading order (LO) of the $(2,2)$ mode, i.e., up to the $\ell=|m|=6$ mode. These higherorder modes are even more important for eccentric orbits than for quasicircular ones [87]. We conclude in Sec. V with a discussion of results and potential future work. Finally, Appendix A provides the coordinate transformation from harmonic to EOB coordinates, Appendix B includes a derivation of the $\mathrm{LO}$ spin-squared contribution to the angular momentum flux, Appendix $\mathrm{C}$ lists the spin contributions to the waveform modes in harmonic coordinates, Appendix D provides some relations for dynamic quantities in the Keplerian parametrization, and Appendix E includes the transformation to tortoise coordinates. We provide our results for the RR force and waveform modes as Mathematica files in the Supplemental Material [88].

\section{A. Notation}

We use the metric signature $(-,+,+,+)$, and use units in which $c=G=1$, but write $c$ explicitly in PN expansions.

We consider an aligned-spin binary with masses $m_{1}$ and $m_{2}$, with $m_{1} \geq m_{2}$, and we define the following constants:

$$
\begin{aligned}
& M=m_{1}+m_{2}, \quad \mu=\frac{m_{1} m_{2}}{M}, \quad \nu=\frac{\mu}{M}, \\
& \delta=\frac{m_{1}-m_{2}}{M}, \quad X_{1}=\frac{m_{1}}{M}, \quad X_{2}=\frac{m_{2}}{M} .
\end{aligned}
$$

In the binary's center of mass, we introduce the canonical phase-space variables $\left(R, \phi, P_{R}, P_{\phi}\right)$, where $R$ is the separation, $\phi$ the azimuthal angle, $P_{R}$ the radial momentum, and $P_{\phi}$ the angular momentum. The total relative momentum $P$ is given by $P^{2}=P_{R}^{2}+P_{\phi}^{2} / R^{2}$. We use the rescaled dimensionless variables

$$
\begin{aligned}
& r=\frac{R}{M}, \quad t=\frac{T}{M}, \quad p_{r}=\frac{P_{r}}{\mu}, \quad p_{\phi}=\frac{P_{\phi}}{M \mu}, \\
& \hat{H}=\frac{H}{\mu}, \quad \hat{S}_{i}=\frac{S_{i}}{M \mu}, \quad \chi_{i}=\frac{S_{i}}{m_{i}^{2}},
\end{aligned}
$$

where the dimensionless quantities are denoted with either a hat or a lowercase letter.

The energy and angular momentum fluxes far away from the binary are denoted by $\Phi_{E}$ and $\Phi_{J}$, respectively, and scale as follows: 


$$
\Phi_{E}=G c^{5} \tilde{\Phi}_{E}, \quad \Phi_{J}=c^{5} \frac{\tilde{\Phi}_{J}}{M},
$$

where quantities with a tilde are the physical dimensionful fluxes. The components of the RR force are denoted by $\mathcal{F}_{r}$ and $\mathcal{F}_{\phi}$, and are scaled similarly to $\Phi_{E}$ and $\Phi_{J}$, respectively.

\section{RADIATION REACTION FORCE}

The RR force accounts for the energy and angular momentum losses by the system, and is added to the right-hand side of the Hamilton equations of motion (EOMs) such that

$$
\begin{aligned}
\dot{r}=\frac{\partial \hat{H}}{\partial p_{r}}, & \dot{p}_{r}=-\frac{\partial \hat{H}}{\partial r}+\mathcal{F}_{r}, \\
\dot{\phi}=\frac{\partial \hat{H}}{\partial p_{\phi}}, & \dot{p}_{\phi}=-\frac{\partial \hat{H}}{\partial \phi}+\mathcal{F}_{\phi},
\end{aligned}
$$

where the leading order of $\mathcal{F}_{r, \phi}$ is of order $1 / c^{5}(2.5 \mathrm{PN})$. From the EOMs, with $\partial H / \partial \phi=0$, the time derivatives of energy and angular momentum are given by

$$
\begin{aligned}
& \dot{E}_{\text {system }}=\frac{d \hat{H}}{d t}=\dot{r} \mathcal{F}_{r}+\dot{\phi} \mathcal{F}_{\phi}, \\
& \dot{J}_{\text {system }}=\frac{d p_{\phi}}{d t}=\mathcal{F}_{\phi} .
\end{aligned}
$$

The energy and angular momentum lost by the system are not equal to the energy and angular momentum fluxes, $\Phi_{E}$ and $\Phi_{J}$, because of additional contributions to $E$ and $J$ due to interactions with the radiation field. The balance equations are modified by Schott terms, as in electrodynamics, that appear as total time derivatives in the balance equations [78]

$$
\begin{gathered}
\dot{E}_{\text {system }}+\dot{E}_{\text {Schott }}+\Phi_{E}=0, \\
\dot{J}_{\text {system }}+\dot{J}_{\text {Schott }}+\Phi_{J}=0 .
\end{gathered}
$$

Substituting the expressions for the energy and angular momentum losses, we obtain

$$
\begin{aligned}
\dot{r} \mathcal{F}_{r}+\dot{\phi} \mathcal{F}_{\phi}+\dot{E}_{\text {Schott }}+\Phi_{E} & =0, \\
\mathcal{F}_{\phi}+\dot{J}_{\text {Schott }}+\Phi_{J} & =0 .
\end{aligned}
$$

The energy and angular momentum fluxes are gauge independent, but the RR force and Schott terms are gauge dependent. This coordinate gauge freedom in the RR force was discussed by Iyer and Will in Refs. [89,90], and by Gopakumar et al. in Ref. [91]. Bini and Damour showed in Ref. [78] how the gauge freedom in $\mathcal{F}$ is related to the freedom in defining the Schott terms.
Note that while we only consider aligned spins in this paper, an extension to precessing spins is straightforward; the RR force $\mathcal{F}$ is added to the EOM for the total momentum $\boldsymbol{p}$, and a RR contribution is added to the spin evolution equations, such that

$$
\begin{aligned}
\frac{d \boldsymbol{r}}{d t} & =\frac{\partial H}{\partial \boldsymbol{p}}, \quad \frac{d \boldsymbol{p}}{d t}=-\frac{\partial H}{\partial \boldsymbol{r}}+\mathcal{F}, \\
\frac{d \boldsymbol{S}_{\mathrm{i}}}{d t} & =\frac{\partial H}{\partial \boldsymbol{S}_{\mathrm{i}}} \times \boldsymbol{S}_{\mathrm{i}}+\dot{\boldsymbol{S}}_{\mathrm{i}}^{\mathrm{RR}} .
\end{aligned}
$$

The balance equations are then given by

$$
\begin{gathered}
\dot{E}_{\text {system }}+\dot{E}_{\text {Schott }}+\Phi_{E}=0, \\
\dot{\boldsymbol{J}}_{\text {system }}+\dot{\boldsymbol{J}}_{\text {Schott }}+\boldsymbol{\Phi}_{J}=0,
\end{gathered}
$$

with

$$
\begin{aligned}
& \dot{E}_{\text {system }}=\dot{\boldsymbol{r}} \cdot \mathcal{F}, \\
& \dot{\boldsymbol{J}}_{\text {system }}=\boldsymbol{r} \times \mathcal{F}+\dot{\boldsymbol{S}}_{1}^{\mathrm{RR}}+\dot{\boldsymbol{S}}_{2}^{\mathrm{RR}} .
\end{aligned}
$$

See, e.g., Refs. [92,93] for more details.

\section{A. Summary of the approach used in this paper for the $R R$ force}

The aim of this paper is to extend the quasicircular $\mathrm{RR}$ force and gravitational modes employed in the SEOBNRV4HM waveform model to eccentric orbits. The Hamilton equations that describe the dynamics of the SEOBNRV4HM model use the following relations between the RR force and the energy flux for quasicircular orbits, which are based on results from Refs. [46,94]:

$$
\begin{aligned}
& \mathcal{F}_{\phi}^{\mathrm{qc}}=-\frac{\Phi_{E}^{\mathrm{qc}}}{\Omega}, \\
& \mathcal{F}_{r}^{\mathrm{qc}}=\mathcal{F}_{\phi}^{\mathrm{qc}} \frac{p_{r}}{p_{\phi}}=-\frac{\Phi_{E}^{\mathrm{qc}} p_{r}}{\Omega p_{\phi}},
\end{aligned}
$$

with $\Omega$ being the (angular) orbital frequency. However, these two relations are only valid for quasicircular orbits and are not consistent for generic orbits, since they use the circular-orbit relation $\Phi_{E}^{\mathrm{qc}}=\Omega \Phi_{J}^{\mathrm{qc}}$ and do not include the Schott terms.

Hence, the approach we use to obtain the RR force is to write a generic ansatz with unknown coefficients for the Schott terms, and calculate the RR force from the fluxes using the balance equations

$$
\begin{aligned}
\mathcal{F}_{\phi} & =-\Phi_{J}-\dot{J}_{\text {Schott }} \\
\dot{r} \mathcal{F}_{r} & =-\Phi_{E}+\dot{\phi} \Phi_{J}-\dot{E}_{\text {Schott }}+\dot{\phi} \dot{J}_{\text {Schott }}
\end{aligned}
$$


Then, we specify the free unknown coefficients in the Schott terms such that the force reduces to the conditions in Eq. (11) in the limit of quasicircular orbits, i.e.,

$$
\begin{aligned}
\mathcal{F}_{\phi} & =-\Phi_{J}+\mathcal{O}\left(\dot{p}_{r}\right)+\mathcal{O}\left(p_{r}^{2}\right), \\
\frac{\mathcal{F}_{r} p_{\phi}}{\mathcal{F}_{\phi} p_{r}} & =1+\mathcal{O}\left(p_{r}^{2}\right),
\end{aligned}
$$

since both $p_{r}$ and $\dot{p}_{r}$ are zero for circular orbits. Finally, we factorize the RR force into the quasicircular part used in SEOBNRV4HM times eccentric corrections

$$
\mathcal{F}_{r}=\mathcal{F}_{r}^{\mathrm{qc}} \mathcal{F}_{r}^{\mathrm{ecc}}, \quad \mathcal{F}_{\phi}=\mathcal{F}_{\phi}^{\mathrm{qc}} \mathcal{F}_{\phi}^{\mathrm{ecc}},
$$

where the quasicircular parts are given by Eq. (11), and the eccentric corrections scale as $\mathcal{F}_{i}^{\text {nc }} \sim 1+\dot{p}_{r}+p_{r}^{2}+\cdots$. In the following subsections, we provide the details of these steps.

\section{B. EOB Hamiltonian and angular momentum}

The EOB Hamiltonian is calculated from an effective Hamiltonian $H_{\text {eff }}$ via the energy map

$$
H_{\mathrm{EOB}}=M \sqrt{1+2 \nu\left(\frac{H_{\mathrm{eff}}}{\mu}-1\right)},
$$

with $H_{\text {eff }}$ given in Refs. $[50,51,56]$. When calculating the $\mathrm{RR}$ force to $2 \mathrm{PN}$, we only need to work with the PN expansion of the EOB Hamiltonian. The nonspinning part to $2 \mathrm{PN}$ order is given by

$$
\begin{aligned}
\hat{H}_{\mathrm{EOB}}^{0}= & \frac{c^{2}}{\nu}+\frac{p^{2}}{2}-\frac{1}{r}+\frac{1}{c^{2}}\left[\frac{(\nu-1) p^{2}}{2 r}-\frac{1+\nu}{8} p^{4}-\frac{p_{r}^{2}}{r}\right. \\
& \left.-\frac{1+\nu}{2 r^{2}}\right]+\frac{1}{c^{4}}\left[\frac{1+\nu+\nu^{2}}{16} p^{6}+\frac{(1+2 \nu) p_{r}^{2}}{r^{2}}\right. \\
& -\frac{\left(1+\nu-3 \nu^{2}\right) p^{2}}{4 r^{2}}+\frac{\left(1+\nu-3 \nu^{2}\right) p^{4}}{8 r} \\
& \left.+\frac{(1+\nu) p^{2} p_{r}^{2}}{2 r}-\frac{1-\nu+\nu^{2}}{2 r^{3}}\right],
\end{aligned}
$$

the LO (1.5PN) spin-orbit part

$$
\hat{H}_{\mathrm{EOB}}^{\mathrm{SO}}=\frac{p_{\phi}}{2 c^{3} r^{3}}\left[\chi_{1}(2+2 \delta-\nu)+\chi_{2}(2-2 \delta-\nu)\right]
$$

and the LO (2PN) spin-spin part

$$
\begin{aligned}
\hat{H}_{\mathrm{EOB}}^{\mathrm{SS}}= & \frac{1}{2 c^{4} r^{3}}\left\{\chi _ { 1 } ^ { 2 } \left[X_{1}^{4}\left(1-\frac{p_{\phi}^{2}}{r}+r p_{r}^{2}\right)-C_{\left.1 \mathrm{ES}^{2} X_{1}^{2}\right]}\right.\right. \\
& +\chi_{2}^{2}\left[X_{2}^{4}\left(1-\frac{p_{\phi}^{2}}{r}+r p_{r}^{2}\right)-C_{\left.2 \mathrm{ES}^{2} X_{2}^{2}\right]}\right. \\
& \left.+2 \chi_{1} \chi_{2}\left[(\nu-1) \nu-\frac{\nu^{2} p_{\phi}^{2}}{r}+\nu^{2} r p_{r}^{2}\right]\right\}
\end{aligned}
$$

where $C_{i \mathrm{ES}^{2}}$ are the spin quadrupole constants, which equal one for BHs.

The orbital frequency expanded to $2 \mathrm{PN}$ is given by

$$
\begin{aligned}
\Omega \equiv & \dot{\phi}=\frac{\partial \hat{H}_{\mathrm{EOB}}}{\partial p_{\phi}}=\frac{p_{\phi}}{r^{2}}+\frac{p_{\phi}}{c^{2}}\left[\frac{\nu-1}{r^{3}}-\frac{(\nu+1) p^{2}}{2 r^{2}}\right]+\frac{1}{2 c^{3} r^{3}}\left[\chi_{1}(2+2 \delta-\nu)+\chi_{1}(2-2 \delta-\nu)\right] \\
& +\frac{p_{\phi}}{c^{4}}\left[\frac{3\left(\nu^{2}+\nu+1\right) p^{4}}{8 r^{2}}+\frac{\left(-3 \nu^{2}+\nu+1\right) p^{2}}{2 r^{3}}+\frac{(\nu+1) p_{r}^{2}}{r^{3}}-\frac{-3 \nu^{2}+\nu+1}{2 r^{4}}\right] \\
& -\frac{p_{\phi}}{c^{4} r^{4}}\left(2 \nu^{2} \chi_{1} \chi_{2}+\chi_{1}^{2} X_{1}^{4}+\chi_{2}^{2} X_{2}^{4}\right) .
\end{aligned}
$$

From the EOM $\dot{p}_{r}=-\partial \hat{H} / \partial r$, we can obtain an expression for $p_{\phi}\left(r, \dot{p}_{r}, p_{r}\right)$,

$$
\begin{aligned}
\frac{p_{\phi}^{2}}{r}= & 1+r^{2} \dot{p}_{r}+\frac{1}{2 c^{2} r}\left[6+(\nu+1) r^{4} \dot{p}_{r}^{2}-(\nu-5) r^{2} \dot{p}_{r}+r p_{r}^{2}\left((\nu+1) r^{2} \dot{p}_{r}+4\right)\right] \\
& -\frac{3 \sqrt{r^{3} \dot{p}_{r}+r}}{2 c^{3} r^{2}}\left[\chi_{1}(2+2 \delta-\nu)+\chi_{2}(2-2 \delta-\nu)\right] \\
& +\frac{1}{8 c^{4} r^{2}}\left[\left(\nu^{2}+5 \nu+1\right) r^{6} \dot{p}_{r}^{3}-\left(\nu^{2}-\nu+1\right) r^{4} p_{r}^{4} \dot{p}_{r}+2(5 \nu+8) r^{4} \dot{p}_{r}^{2}-\left(\nu^{2}+7 \nu-63\right) r^{2} \dot{p}_{r}\right. \\
& \left.-24(\nu-3)+2\left(8-24 \nu+3 \nu r^{4} \dot{p}_{r}^{2}\right) r p_{r}^{2}+2\left(\nu^{2}+\nu+3\right) p_{r}^{2} r^{3} \dot{p}_{r}\right] \\
& +\frac{1}{2 c^{4} r^{2}}\left\{\chi_{1}^{2}\left[3 C_{1 \mathrm{ES}^{2}} X_{1}^{2}+X_{1}^{4}\left(1+4 r^{2} \dot{p}_{r}-2 r p_{r}^{2}\right)\right]+\chi_{2}^{2}\left[3 C_{2 \mathrm{ES}^{2}} X_{2}^{2}+X_{2}^{4}\left(1+4 r^{2} \dot{p}_{r}-2 r p_{r}^{2}\right)\right]\right. \\
& \left.+\nu \chi_{1} \chi_{2}\left[2\left(\nu+4 \nu r^{2} \dot{p}_{r}+3\right)-4 \nu r p_{r}^{2}\right]\right\},
\end{aligned}
$$


which we use to express the noncircular part of the RR force and modes in terms of $p_{r}$ and $\dot{p}_{r}$. It will also be useful below, when taking the circular-orbit limit, to have an expression for $p_{\phi}$ as a function of $r$ for circular orbits. Setting $\dot{p}_{r}=0=p_{r}$ in the previous equation yields

$$
\begin{aligned}
\frac{p_{\phi}^{2}}{r} \stackrel{\text { circ }}{=} & 1+\frac{3}{c^{2} r}+\frac{3(3-\nu)}{c^{4} r^{2}} \\
& -\frac{3}{2 c^{3} r^{3 / 2}}\left[\chi_{1}(-2 \delta+\nu-2)+\chi_{2}(2 \delta+\nu-2)\right] \\
& +\frac{1}{2 c^{4} r^{2}}\left[\chi_{1}^{2}\left(3 C_{1 \mathrm{ES}^{2}} X_{1}^{2}+X_{1}^{4}\right)+2 \nu \chi_{1} \chi_{2}(3+\nu)\right. \\
& \left.+\chi_{2}^{2}\left(3 C_{2 \mathrm{ES}^{2}} X_{2}^{2}+X_{2}^{4}\right)\right] .
\end{aligned}
$$

$$
\begin{aligned}
\left\langle\Phi_{E}^{\text {tail }}\right\rangle= & \frac{128 \pi \nu^{2}}{5 c^{3}} x^{13 / 2}\left[1+\frac{2335}{192} e^{2}+\frac{42955}{768} e^{4}\right. \\
& \left.+\frac{6204647}{36864} e^{6}+\mathcal{O}\left(e^{8}\right)\right] \\
\left\langle\Phi_{J}^{\text {tail }}\right\rangle= & \frac{128 \pi \nu^{2}}{5 c^{3}} x^{5}\left[1+\frac{209}{32} e^{2}+\frac{2415}{128} e^{4}+\frac{730751}{18432} e^{6}\right. \\
& \left.+\mathcal{O}\left(e^{8}\right)\right]
\end{aligned}
$$

where $x \equiv \Omega^{2 / 3}$. The eccentricity $e$ in these equations is defined using the Keplerian parametrization, which is given by

$$
r=\frac{1}{u_{p}(1+e \cos \chi)}
$$

\section{Energy and angular momentum fluxes}

The energy and angular momentum fluxes for nonspinning binaries were derived to $3 \mathrm{PN}$ order in harmonic and Arnowitt-Deser-Misner (ADM) coordinates in Refs. [95-97]. The 2PN instantaneous part of the fluxes for nonspinning bodies is given in EOB coordinates in Appendix A of Ref. [78]. The leading order reads

$$
\begin{gathered}
\Phi_{E}^{\mathrm{inst}}=\frac{8 \nu^{2}}{15 r^{4}}\left(12 p^{2}-11 p_{r}^{2}\right)+\mathcal{O}\left(\frac{1}{c^{2}}\right), \\
\Phi_{J}^{\mathrm{inst}}=\frac{8 \nu^{2}}{5 r^{3}} p_{\phi}\left(2 p^{2}-3 p_{r}^{2}+\frac{2}{r}\right)+\mathcal{O}\left(\frac{1}{c^{2}}\right) .
\end{gathered}
$$

The hereditary contributions to the fluxes can be expressed as an infinite sum over Bessel functions $[79,96]$ that can be evaluated numerically or resummed analytically $[31,98]$. Here, we follow the method from Ref. [79] to obtain the LO tail part (1.5 PN) of the orbitaveraged fluxes in an eccentricity expansion, and we extend their derivation to $\mathcal{O}\left(e^{6}\right)$, which yields ${ }^{2}$

\footnotetext{
${ }^{2}$ Calculating the tail contribution to the fluxes is similar to that for the waveform modes (see Sec. IV B) except for using the integrals [99]

$$
\begin{aligned}
\Phi_{E}^{\text {tail }}= & \frac{4}{5} I_{i j}^{(3)} \int_{0}^{\infty} d \tau I_{i j}^{(5)}(t-\tau) \ln \left(\frac{\tau}{b}\right), \\
\Phi_{J}^{\text {tail }}= & \frac{4}{5} \epsilon_{z i j}\left[I_{i l}^{(2)} \int_{0}^{\infty} d \tau I_{j l}^{(5)}(t-\tau) \ln \left(\frac{\tau}{b}\right)\right. \\
& \left.+I_{j l}^{(3)} \int_{0}^{\infty} d \tau I_{i l}^{(4)}(t-\tau) \ln \left(\frac{\tau}{b}\right)\right],
\end{aligned}
$$

where $I_{i j}$ is the mass quadrupole moment and $b=2 r_{0} \mathrm{e}^{-11 / 12}$ with $r_{0}$ a gauge parameter.
}

where $u_{p}$ is the inverse semilatus rectum and $\chi$ is the relativistic anomaly.

Since we are not using the adiabatic approximation and are not working with orbit-averaged fluxes, we can obtain an approximate expression for the tail contribution to the fluxes by writing an ansatz in terms of $\left(r, p_{r}, p_{\phi}\right)$ in a $p_{r}$ expansion of the form

$$
\begin{aligned}
& \Phi_{E}^{\text {tail }}=\frac{128 \pi \nu^{2} p_{\phi}}{5 c^{3} r^{4}}\left[\frac{1}{r^{3}}+c_{1} \frac{p_{r}^{2}}{r^{2}}+c_{2} \frac{p_{r}^{4}}{r}+c_{3} p_{r}^{6}+\mathcal{O}\left(p_{r}^{8}\right)\right], \\
& \Phi_{J}^{\text {tail }}=\frac{128 \pi \nu^{2}}{5 c^{3} r^{2}}\left[\frac{1}{r^{3}}+c_{4} \frac{p_{r}^{2}}{r^{2}}+c_{5} \frac{p_{r}^{4}}{r}+c_{6} p_{r}^{6}+\mathcal{O}\left(p_{r}^{8}\right)\right],
\end{aligned}
$$

calculate the average of that ansatz in terms of $(e, x)$ (see Appendix D), and then match it to the average flux in Eq. (24) to determine the unknowns $c_{n}$. This yields

$$
\begin{aligned}
& \Phi_{E}^{\text {tail }}=\frac{128 \pi \nu^{2} p_{\phi}}{5 c^{3} r^{4}}\left[\frac{1}{r^{3}}+\frac{415 p_{r}^{2}}{96 r^{2}}+\frac{5 p_{r}^{4}}{288 r}-\frac{73 p_{r}^{6}}{11520}+\mathcal{O}\left(p_{r}^{8}\right)\right], \\
& \Phi_{J}^{\text {tail }}=\frac{128}{5 c^{3} r^{2}} \pi \nu^{2}\left[\frac{1}{r^{3}}+\frac{49 p_{r}^{2}}{16 r^{2}}-\frac{49 p_{r}^{6}}{5760}+\mathcal{O}\left(p_{r}^{8}\right)\right] .
\end{aligned}
$$

The LO (1.5PN) SO fluxes for generic orbits and generic spins were derived in Refs. [92,100]. (The next-to-leadingorder (NLO) SO energy flux was derived in Ref. [101].) It should be noted that Ref. [100] used the Tulczyjew-Dixon (covariant) spin supplementary condition (SSC) [102-105], while Ref. [92] used the Newton-Wigner (NW), or canonical, SSC [106,107]. In this paper, we use the NW SSC since we are working in a canonical Hamiltonian formulation of the spinning two-body dynamics $[108,109]$. Changing the velocities in Eq. (17) of Ref. [92] to momenta, which involves spin-orbit terms, the aligned-spin fluxes reduce to 


$$
\begin{aligned}
\Phi_{E}^{\mathrm{SO}}= & \frac{4 \nu^{2} p_{\phi}}{15 c^{3} r^{6}}\left\{\chi _ { 1 } \left[p^{2}(36 \nu-37-37 \delta)+\frac{4(9+9 \delta-4 \nu)}{r}\right.\right. \\
& \left.\left.+9 p_{r}^{2}(3+3 \delta-2 \nu)\right]+1 \leftrightarrow 2\right\}, \\
\Phi_{J}^{\mathrm{SO}}= & \frac{4 \nu^{2}}{15 c^{3} r^{3}}\left\{\chi _ { 1 } \left[p^{4}(8 \nu-9 \delta-9)+\frac{9+9 \delta-4 \nu}{r^{2}}\right.\right. \\
& +(9+9 \delta-24 \nu) p^{2} p_{r}^{2}-(17+17 \delta+6 \nu) \frac{p_{r}^{2}}{r} \\
& \left.\left.+15 \nu p_{r}^{4}+(11+11 \delta+10 \nu) \frac{p^{2}}{r}\right]+1 \leftrightarrow 2\right\} . \quad(28)
\end{aligned}
$$

For the LO (2PN) SS contributions, the LO $\operatorname{spin}_{1}-\mathrm{spin}_{2}$ energy and angular momentum fluxes in harmonic coordinates were derived in Refs. [93,100], while the spinsquared energy flux was derived in Refs. [110,111], and we obtain in Appendix B the spin-squared angular momentum flux. Transforming from harmonic to EOB coordinates, using the transformations in Appendix A, we get the following SS contributions to the fluxes for aligned spins:

$$
\begin{aligned}
\Phi_{E}^{\mathrm{SS}}= & \frac{\nu^{2}}{15 c^{4} r^{6}}\left\{\chi _ { 1 } ^ { 2 } \left[C_{1 \mathrm{ES}^{2}}(\delta-2 \nu+1)\left(144 p^{2}-156 p_{r}^{2}\right)\right.\right. \\
& +3\left(96 \nu \delta-47 \delta-96 \nu^{2}+190 \nu-47\right) p^{2} \\
& \left.+\left(149 \delta-280 \nu \delta+280 \nu^{2}-578 \nu+149\right) p_{r}^{2}\right] \\
& +\nu \chi_{1} \chi_{2}\left[10(28 \nu-33) p_{r}^{2}-6(48 \nu-47) p^{2}\right] \\
& +1 \leftrightarrow 2\}, \\
\Phi_{J}^{S S}= & \frac{\nu^{2} p_{\phi}}{5 c^{4} r^{5}}\left\{\chi _ { 1 } ^ { 2 } \left[\left(32 \nu \delta-15 \delta-32 \nu^{2}+62 \nu-15\right) \frac{1}{r}\right.\right. \\
& +\left(\delta-2 \delta \nu+2 \nu^{2}-4 \nu+1\right)\left(22 p_{r}^{2}-20 p^{2}\right) \\
& \left.+C_{1 \mathrm{ES}}(\delta-2 \nu+1)\left(12 p^{2}-30 p_{r}^{2}+\frac{24}{r}\right)\right] \\
& +\nu \chi_{1} \chi_{2}\left[\frac{2(23-16 \nu)}{r}-8(5 \nu-3) p^{2}\right. \\
& \left.\left.+4(11 \nu-15) p_{r}^{2}\right]+1 \leftrightarrow 2\right\} .
\end{aligned}
$$

The total 2PN energy and angular momentum fluxes are the sum of all the above contributions, i.e.,

$$
\begin{aligned}
& \Phi_{E}=\Phi_{E}^{\mathrm{inst}}+\Phi_{E}^{\mathrm{tail}}+\Phi_{E}^{\mathrm{SO}}+\Phi_{E}^{\mathrm{SS}}, \\
& \Phi_{J}=\Phi_{J}^{\mathrm{inst}}+\Phi_{J}^{\mathrm{tail}}+\Phi_{J}^{\mathrm{SO}}+\Phi_{J}^{\mathrm{SS}} .
\end{aligned}
$$

\section{Ansatz for the Schott terms}

As an ansatz for the Schott terms $E_{\text {Schott }}$ and $J_{\text {Schott }}$, we consider

$$
\begin{aligned}
J_{\text {Schott }}^{\text {inst }}= & \frac{\nu^{2} p_{r} p_{\phi}}{r^{2}}\left[\alpha_{1}+\frac{1}{c^{2}}\left(\alpha_{2} p_{r}^{2}+\alpha_{3} p^{2}+\frac{\alpha_{4}}{r}\right)\right. \\
& +\frac{1}{c^{4}}\left(\alpha_{5} p_{r}^{4}+\alpha_{6} p^{2} p_{r}^{2}+\alpha_{7} \frac{p_{r}^{2}}{r}+\alpha_{8} p^{4}+\alpha_{9} \frac{p^{2}}{r}\right. \\
& \left.\left.+\frac{\alpha_{10}}{r^{2}}\right)\right], \\
E_{\text {Schott }}^{\text {inst }}= & \frac{\nu^{2} p_{r}}{r^{2}}\left[\beta_{1} p_{r}^{2}+\beta_{2} p^{2}+\frac{\beta_{3}}{r}+\frac{1}{c^{2}}\left(\beta_{4} p_{r}^{4}+\beta_{5} p^{2} p_{r}^{2}\right.\right. \\
& \left.+\beta_{6} \frac{p_{r}^{2}}{r}+\beta_{7} p^{4}+\beta_{8} \frac{p^{2}}{r}+\frac{\beta_{9}}{r^{2}}\right) \\
& +\frac{1}{c^{4}}\left(\beta_{10} p_{r}^{6}+\beta_{11} p^{2} p_{r}^{4}+\beta_{12} \frac{p_{r}^{4}}{r}+\beta_{13} p^{4} p_{r}^{2}\right. \\
& +\beta_{14} \frac{p^{2} p_{r}^{2}}{r}+\beta_{15} \frac{p_{r}^{2}}{r^{2}}+\beta_{16} p^{6}+\beta_{17} \frac{p^{4}}{r} \\
& \left.\left.+\beta_{18} \frac{p^{2}}{r^{2}}+\frac{\beta_{19}}{r^{3}}\right)\right] .
\end{aligned}
$$

Note that this ansatz for $E_{\text {Schott }}$ is more general than the one used in Eq. (4.4) of Ref. [78], since we found that such an ansatz is needed for the RR force to satisfy the conditions in Eq. (11).

For the LO tail, we use the ansatz

$$
\begin{aligned}
J_{\text {Schott }}^{\text {tail }} & =\frac{\pi \nu^{2} p_{r}}{c^{3} r^{2}}\left(\lambda_{1} p_{r}^{2}+\lambda_{2} p^{2}+\frac{\lambda_{3}}{r}\right), \\
E_{\text {Schott }}^{\text {tail }} & =\frac{\pi \nu^{2} p_{\phi} p_{r}}{c^{3} r^{4}}\left(\lambda_{4} p_{r}^{2}+\lambda_{5} p^{2}+\frac{\lambda_{6}}{r}\right),
\end{aligned}
$$

while for the LO SO part,

$$
\begin{aligned}
J_{\text {Schott }}^{\text {SO }}= & \frac{\nu^{2} p_{r}}{c^{3} r^{2}}\left[\chi_{1}\left(\sigma_{1} p_{r}^{2}+\sigma_{2} p^{2}+\frac{\sigma_{3}}{r}\right)\right. \\
& \left.+\chi_{2}\left(\sigma_{4} p_{r}^{2}+\sigma_{5} p^{2}+\frac{\sigma_{6}}{r}\right)\right], \\
E_{\text {Schott }}^{\text {SO }}= & \frac{\nu^{2} p_{r} p_{\phi}}{c^{3} r^{4}}\left[\chi_{1}\left(\sigma_{7} p_{r}^{2}+\sigma_{8} p^{2}+\frac{\sigma_{9}}{r}\right)\right. \\
& \left.+\chi_{2}\left(\sigma_{10} p_{r}^{2}+\sigma_{11} p^{2}+\frac{\sigma_{12}}{r}\right)\right],
\end{aligned}
$$

and for the SS part, 


$$
\begin{aligned}
J_{\text {Schott }}^{\text {SS }}= & \frac{\nu^{2} p_{\phi} p_{r}}{c^{4} r^{4}}\left[\chi_{1}^{2}\left(\zeta_{1}+C_{1 \mathrm{ES}^{2}} \zeta_{2}\right)\right. \\
& \left.+\chi_{1} \chi_{2} \zeta_{3}+\chi_{2}^{2}\left(\zeta_{4}+C_{2 \mathrm{ES}^{2}} \zeta_{5}\right)\right], \\
E_{\mathrm{Schott}}^{\mathrm{SS}}= & \frac{\nu^{2} p_{r}}{c^{4} r^{4}}\left[\chi_{1}^{2}\left(\zeta_{6} p_{r}^{2}+\zeta_{7} p^{2}+\frac{\zeta_{8}}{r}\right)\right. \\
& +\chi_{1}^{2} C_{1 \mathrm{ES}^{2}}\left(\zeta_{9} p_{r}^{2}+\zeta_{10} p^{2}+\frac{\zeta_{11}}{r}\right) \\
& +\chi_{1} \chi_{2}\left(\zeta_{12} p_{r}^{2}+\zeta_{13} p^{2}+\frac{\zeta_{14}}{r}\right) \\
& +\chi_{2}^{2}\left(\zeta_{15} p_{r}^{2}+\zeta_{16} p^{2}+\frac{\zeta_{17}}{r}\right) \\
& \left.+\chi_{2}^{2} C_{2 \mathrm{ES}^{2}}\left(\zeta_{18} p_{r}^{2}+\zeta_{19} p^{2}+\frac{\zeta_{20}}{r}\right)\right] .
\end{aligned}
$$

The total energy and angular momentum Schott terms are the sum of the above contributions, i.e.,

$$
\begin{aligned}
& J_{\text {Schott }}=J_{\text {Schott }}^{\text {inst }}+J_{\text {Schott }}^{\text {tail }}+J_{\text {Schott }}^{\text {SO }}+J_{\text {Schott }}^{\text {SS }}, \\
& E_{\text {Schott }}=E_{\text {Schott }}^{\text {inst }}+E_{\text {Schott }}^{\text {tail }}+E_{\text {Schott }}^{\text {SO }}+E_{\text {Schott }}^{\text {SS }} .
\end{aligned}
$$

Note that when taking the time derivative of these Schott terms using the EOMs, the LO nonspinning part contributes to the LO SO and SS parts of the RR force.

\section{E. Solving for the eccentric-orbits RR force}

Using the fluxes and the Schott terms, the RR force can be calculated from the balance equations (12), which fix some of the unknowns in the ansatz for the Schott terms. The remaining unknowns can be determined by requiring that the RR force satisfies the conditions (13) in the circular-orbits limit.

\section{Leading order}

At leading order, calculating the RR force with the ansatz in Eqs. (31) and expanding in $p_{r}$ gives

$$
\begin{aligned}
\mathcal{F}_{r}= & \frac{\nu^{2}}{5 p_{r} r^{3}}\left(p^{2}-\frac{1}{r}\right)\left[p^{2}\left(5 \alpha_{1}-5 \beta_{2}+16\right)-5 \frac{\beta_{3}}{r}\right] \\
& +\mathcal{O}\left(p_{r}\right) .
\end{aligned}
$$

Requiring that the $1 / p_{r}$ term is zero leads to the solution

$$
\beta_{3}=0, \quad \alpha_{1}=\frac{1}{5}\left(5 \beta_{2}-16\right) .
$$

Expanding $\mathcal{F}_{r} p_{\phi} /\left(\mathcal{F}_{\phi} p_{r}\right)-1$ in $p_{r}$ yields

$$
\begin{aligned}
\frac{\mathcal{F}_{r} p_{\phi}}{\mathcal{F}_{\phi} p_{r}}-1= & \frac{9\left(5 \beta_{1}-8\right) p^{2}-\left(45 \beta_{1}+30 \beta_{2}+88\right) / r}{15 \beta_{2} p^{2}+3\left(32-5 \beta_{2}\right) / r} \\
& +\mathcal{O}\left(p_{r}^{2}\right) .
\end{aligned}
$$

Requiring that the first term in that series expansion is zero gives the solution

$$
\beta_{1}=\frac{8}{5}, \quad \beta_{2}=-\frac{16}{3}, \quad \alpha_{1}=-\frac{128}{15} .
$$

With that solution, we obtain the LO RR force

$$
\begin{aligned}
& \mathcal{F}_{\phi}^{\mathrm{LO}}=\frac{8 \nu^{2}}{15 r^{3}} p_{\phi}\left(10 p^{2}-39 p_{r}^{2}-\frac{22}{r}\right), \\
& \mathcal{F}_{r}^{\mathrm{LO}}=-\frac{16 \nu^{2}}{15 r^{3}} p_{r}\left(-5 p^{2}+12 p_{r}^{2}+\frac{11}{r}\right) .
\end{aligned}
$$

This force satisfies the conditions in Eq. (13) for circular orbits since

$$
\begin{aligned}
\mathcal{F}_{\phi}^{\mathrm{LO}} & =-\Phi_{J}^{\mathrm{LO}}-\frac{128}{15 r^{3}} p_{\phi} \nu^{2}\left(2 p_{r}^{2}-\dot{p}_{r} r\right), \\
\frac{\mathcal{F}_{r}^{\mathrm{LO}} p_{\phi}}{\mathcal{F}_{\phi}^{\mathrm{LO}} p_{r}} & =1+\frac{15 p_{r}^{2}}{10 p^{2}-39 p_{r}^{2}-22 / r} .
\end{aligned}
$$

\section{2. $1 P N$}

Following the same steps as above, we obtain the following solution for the unknowns at $1 \mathrm{PN}$ :

$$
\begin{aligned}
& \beta_{8}=\frac{1}{15}\left(371-15 \alpha_{3}+268 \nu\right), \\
& \alpha_{2}=\frac{1}{35}\left(35 \beta_{5}+108 \nu-22\right), \\
& \beta_{6}=\frac{2}{315}\left(105 \alpha_{3}-1760 \nu+1743\right), \\
& \beta_{9}=-\frac{32}{105}(4 \nu-1), \\
& \beta_{7}=\alpha_{3}+\frac{2}{35}(4 \nu+93), \\
& \alpha_{4}=\frac{893}{21}-\alpha_{3}+\frac{2924 \nu}{105},
\end{aligned}
$$

with three arbitrary coefficients out of nine coefficients at that order. To simplify the resulting expressions for the RR force, we choose to set all arbitrary coefficients to zero, i.e., $\alpha_{3}=\beta_{4}=\beta_{5}=0$, which yields the following $1 \mathrm{PN}$ contribution to the RR force:

$$
\begin{aligned}
\mathcal{F}_{\phi}^{1 \mathrm{PN}}= & \frac{\nu^{2} p_{\phi}}{105 c^{2} r^{3}}\left[18(4 \nu+93) p_{r}^{2} p^{2}-6(4 \nu+93) p^{4}\right. \\
& +180(7 \nu-5) p_{r}^{4}+(9780 \nu+19198) \frac{p_{r}^{2}}{r} \\
& \left.-(484 \nu+3833) \frac{p^{2}}{r}+\frac{1684 \nu+6213}{r^{2}}\right], \\
\mathcal{F}_{r}^{1 \mathrm{PN}}= & \frac{\nu^{2} p_{r}}{105 c^{2} r^{3}}\left[180(7 \nu-5) p_{r}^{4}-6(4 \nu+93) p^{4}\right. \\
& +4(691 \nu+3958) \frac{p_{r}^{2}}{r}-198(6 \nu-13) p_{r}^{2} p^{2} \\
& \left.-(484 \nu+3833) \frac{p^{2}}{r}+\frac{1684 \nu+6213}{r^{2}}\right] .
\end{aligned}
$$




\section{LO tail}

Solving for the unknowns at the LO tail contribution leads to the solution

$\lambda_{2}=-\frac{334}{15}, \quad \lambda_{3}=\frac{128}{5}, \quad \lambda_{4}=-\frac{334}{15}+\lambda_{1}$,

$\lambda_{5}=-\frac{334}{15}, \quad \lambda_{6}=\frac{128}{5}$,

with either $\lambda_{1}$ or $\lambda_{4}$ arbitrary. Choosing $\lambda_{1}=0$, the tail contribution to the RR forces becomes

$$
\begin{aligned}
& \mathcal{F}_{\phi}^{\text {tail }}=\frac{\pi \nu^{2}}{c^{3} r^{2}}\left(\frac{334 p^{4}}{15 r}-\frac{718 p^{2}}{15 r^{2}}-\frac{334 p^{2} p_{r}^{2}}{5 r}-\frac{308 p_{r}^{2}}{15 r^{2}}+\frac{49 p_{r}^{6}}{225}\right), \\
& \mathcal{F}_{r}^{\text {tail }}=\frac{\pi \nu^{2} p_{\phi} p_{r}}{c^{3} r^{4}}\left(\frac{334 p^{2}}{15 r}-\frac{p_{r}^{4}}{18}-\frac{7034 p_{r}^{2}}{45 r}-\frac{718}{15 r^{2}}\right)
\end{aligned}
$$

\section{LO spin orbit}

At LO SO, we obtain the solution

$$
\begin{aligned}
\sigma_{5} & =\frac{1}{15}\left(-36 \delta-32 \nu+15 \sigma_{11}+36\right), \\
\sigma_{6} & =\frac{1}{15}\left(-68 \delta-40 \nu+15 \sigma_{12}+68\right), \\
\sigma_{2} & =\frac{1}{15}\left(36 \delta-32 \nu+15 \sigma_{8}+36\right), \\
\sigma_{3} & =\frac{1}{15}\left(68 \delta-40 \nu+15 \sigma_{9}+68\right), \\
\sigma_{12} & =\frac{8}{15}(4 \delta+3 \nu-4), \\
\sigma_{9} & =-\frac{8}{15}(4 \delta-3 \nu+4), \\
\sigma_{4} & =\frac{1}{15}\left(-6 \delta+44 \nu+15 \sigma_{10}+6\right), \\
\sigma_{1} & =\frac{1}{15}\left(6 \delta+44 \nu+15 \sigma_{7}+6\right), \\
\sigma_{11} & =-\frac{2}{15}(9 \delta+22 \nu-9), \\
\sigma_{8} & =\frac{2}{15}(9 \delta-22 \nu+9),
\end{aligned}
$$

where either $\sigma_{1}$ or $\sigma_{7}$ is arbitrary, and either $\sigma_{4}$ or $\sigma_{10}$ is arbitrary. Choosing $\sigma_{7}=\sigma_{10}=0$, we obtain

$$
\begin{aligned}
\mathcal{F}_{\phi}^{\mathrm{SO}}= & \frac{2 \nu^{2}}{15 c^{3} r^{3}} \chi_{1}\left[p^{4}(22 \nu-9 \delta-9)+5(3+3 \delta+16 \nu) p_{r}^{4}\right. \\
& +(66 \nu-23 \delta-23) \frac{p_{r}^{2}}{r}+(179+179 \delta-146 \nu) \frac{p^{2}}{r} \\
& \left.+6(9+9 \delta-22 \nu) p^{2} p_{r}^{2}\right]+1 \leftrightarrow 2 .
\end{aligned}
$$

$$
\begin{aligned}
\mathcal{F}_{r}^{\mathrm{SO}}= & \frac{2 \nu^{2} p_{r} p_{\phi}}{15 c^{3} r^{5}} \chi_{1}\left[\frac{179 \delta-146 \nu+179}{r}+(22 \nu-9 \delta-9) p^{2}\right. \\
& \left.-5(3+3 \delta+16 \nu) p_{r}^{2}\right]+1 \leftrightarrow 2 .
\end{aligned}
$$

\section{2PN no spin}

At 2PN, we obtain

$$
\begin{aligned}
& \alpha_{6}=\beta_{13}-\frac{463 \nu^{2}}{63}-\frac{1909 \nu}{315}+\frac{922}{315}, \\
& \beta_{15}=-\frac{2 \beta_{18}}{3}+\frac{1060 \nu^{2}}{189}-\frac{12116 \nu}{189}-\frac{347236}{2835}, \\
& \beta_{16}=-\beta_{17}-\beta_{18}-\frac{5276 \nu^{2}}{315}-\frac{109609 \nu}{630}+\frac{9175}{378}, \\
& \beta_{14}=\alpha_{7}-\frac{2 \beta_{17}}{3}-\frac{2 \beta_{18}}{3}+\frac{244 \nu^{2}}{45}-\frac{12466 \nu}{135}-\frac{28204}{2835}, \\
& \beta_{19}=-\frac{640 \nu^{2}}{189}+\frac{416 \nu}{35}-\frac{2608}{945}, \\
& \alpha_{8}=-\beta_{17}-\beta_{18}-\frac{5018 \nu^{2}}{315}-\frac{105491 \nu}{630}+\frac{52223}{1890}, \\
& \alpha_{10}=\beta_{18}-\frac{92 \nu^{2}}{9}-\frac{309 \nu}{5}-\frac{10019}{315}, \\
& \alpha_{9}=\beta_{17}-\frac{78 \nu^{2}}{7}+\frac{1201 \nu}{90}+\frac{5711}{126},
\end{aligned}
$$

with eight arbitrary coefficients out of 16. Choosing $\alpha_{5}=$ $\alpha_{7}=\beta_{10}=\beta_{11}=\beta_{12}=\beta_{13}=\beta_{17}=\beta_{18}=0$ yields

$$
\begin{aligned}
\mathcal{F}_{\phi}^{2 \mathrm{PN}}= & \frac{\nu^{2} p_{\phi}}{c^{4} r^{3}}\left\{\left(\frac{5276 \nu^{2}}{315}+\frac{109609 \nu}{630}-\frac{9175}{378}\right) p^{6}+\left(\frac{1519}{54}-\frac{9964 \nu^{2}}{315}-\frac{100847 \nu}{630}\right) \frac{p^{4}}{r}+\left(\frac{3512 \nu^{2}}{315}-\frac{4234 \nu}{45}+\frac{3355}{21}\right) \frac{p^{2}}{r^{2}}\right. \\
& +\left(\frac{9175}{126}-\frac{5276 \nu^{2}}{105}-\frac{109609 \nu}{210}\right) p^{4} p_{r}^{2}+\left(\frac{104296}{945}-\frac{15121 \nu^{2}}{105}-\frac{254732 \nu}{315}\right) \frac{p_{r}^{2} p^{2}}{r}+\left(\frac{52}{3}-\frac{152 \nu^{2}}{9}-\frac{310 \nu}{9}\right) p_{r}^{6} \\
& +\left(-\frac{185 \nu^{2}}{63}+\frac{2171 \nu}{63}-\frac{269}{63}\right) p_{r}^{4} p^{2}+\left(\frac{4112}{35}-\frac{278 \nu^{2}}{45}+\frac{344 \nu}{35}\right) \frac{p_{r}^{4}}{r}+\left(-\frac{1604 \nu^{2}}{315}-\frac{2054 \nu}{7}-\frac{8803}{21}\right) \frac{p_{r}^{2}}{r^{2}} \\
& \left.+\left(\frac{8 \nu^{2}}{15}+\frac{9728 \nu}{315}-\frac{190244}{2835}\right) \frac{1}{r^{3}}\right\}
\end{aligned}
$$




$$
\begin{aligned}
\mathcal{F}_{r}^{2 \mathrm{PN}}= & \frac{\nu^{2} p_{r}}{c^{4} r^{3}}\left\{\left(\frac{5276 \nu^{2}}{315}+\frac{109609 \nu}{630}-\frac{9175}{378}\right) p^{6}+\left(\frac{1519}{54}-\frac{9964 \nu^{2}}{315}-\frac{100847 \nu}{630}\right) \frac{p^{4}}{r}+\left(\frac{3512 \nu^{2}}{315}-\frac{4234 \nu}{45}+\frac{3355}{21}\right) \frac{p^{2}}{r^{2}}\right. \\
& +\left(\frac{9713}{126}-\frac{2129 \nu^{2}}{45}-\frac{350537 \nu}{630}\right) p^{4} p_{r}^{2}+\left(\frac{26459}{945}-\frac{1745 \nu^{2}}{63}-\frac{449227 \nu}{315}\right) \frac{p_{r}^{2} p^{2}}{r}+\left(\frac{52}{3}-\frac{152 \nu^{2}}{9}-\frac{310 \nu}{9}\right) p_{r}^{6} \\
& +\left(\frac{293 \nu^{2}}{21}+\frac{1447 \nu}{21}-\frac{1361}{63}\right) p_{r}^{4} p^{2}+\left(\frac{41612}{315}-\frac{1894 \nu^{2}}{45}+\frac{28648 \nu}{315}\right) \frac{p_{r}^{4}}{r}+\left(\frac{34528 \nu^{2}}{945}-\frac{481624 \nu}{945}-\frac{18761}{45}\right) \frac{p_{r}^{2}}{r^{2}} \\
& \left.+\left(\frac{8 \nu^{2}}{15}+\frac{9728 \nu}{315}-\frac{190244}{2835}\right) \frac{1}{r^{3}}\right\} .
\end{aligned}
$$

At LO SS, we obtain the unique solution

\section{LO spin-spin}

$$
\begin{aligned}
& \zeta_{1}=\frac{1}{30}\left[\delta(73-128 \nu)+128 \nu^{2}-274 \nu+73\right], \quad \zeta_{2}=-\frac{42}{5}(\delta-2 \nu+1), \quad \zeta_{3}=\frac{2}{15} \nu(64 \nu-261), \\
& \zeta_{4}=\frac{1}{30}\left[\delta(128 \nu-73)+128 \nu^{2}-274 \nu+73\right], \quad \zeta_{5}=\frac{42}{5}(\delta+2 \nu-1), \\
& \zeta_{6}=-\frac{74}{15}\left(-2 \delta \nu+\delta+2 \nu^{2}-4 \nu+1\right), \quad \zeta_{7}=\frac{1}{10}\left[\delta(43-80 \nu)+80 \nu^{2}-166 \nu+43\right], \\
& \zeta_{8}=0, \quad \zeta_{9}=2(\delta-2 \nu+1), \quad \zeta_{10}=-6(\delta-2 \nu+1), \quad \zeta_{11}=0, \\
& \zeta_{12}=\frac{8}{15}(15-37 \nu) \nu, \quad \zeta_{13}=\frac{2}{5} \nu(40 \nu-63), \quad \zeta_{14}=0, \quad \zeta_{15}=-\frac{74}{15}\left[\delta(2 \nu-1)+2 \nu^{2}-4 \nu+1\right], \\
& \zeta_{16}=\frac{1}{10}\left[\delta(80 \nu-43)+80 \nu^{2}-166 \nu+43\right], \quad \zeta_{17}=0, \quad \zeta_{18}=-2(\delta+2 \nu-1), \\
& \zeta_{19}=6(\delta+2 \nu-1), \quad \zeta_{20}=0 .
\end{aligned}
$$

With that solution, we get

$$
\begin{aligned}
\mathcal{F}_{\phi}^{\mathrm{SS}}= & \frac{\nu^{2} p_{\phi}}{30 c^{4} r^{5}}\left\{\chi _ { 1 } ^ { 2 } \left[24 X_{1}^{2} C_{1 \mathrm{ES}^{2}}\left(15 p^{2}-90 p_{r}^{2}-\frac{49}{r}\right)+\left(361 \delta-632 \nu \delta+632 \nu^{2}-1354 \nu+361\right) p_{r}^{2}\right.\right. \\
& \left.+\left(400 \nu \delta-209 \delta-400 \nu^{2}+818 \nu-209\right) p^{2}+\left(355 \delta-704 \nu \delta+704 \nu^{2}-1414 \nu+355\right) \frac{1}{r}\right] \\
& \left.+\nu \chi_{1} \chi_{2}\left[(378-400 \nu) p^{2}+(632 \nu-2250) p_{r}^{2}+\frac{704 \nu-1182}{r}\right]+1 \leftrightarrow 2\right\} \\
\mathcal{F}_{r}^{\mathrm{SS}}= & \frac{\nu^{2} p_{r}}{30 c^{4} r^{5}}\left\{\chi _ { 1 } ^ { 2 } \left[24 X_{1}^{2} C_{1 \mathrm{ES}^{2}}\left(15 p^{2}-55 p_{r}^{2}-\frac{49}{r}\right)+\left(301 \delta-512 \delta \nu+512 \nu^{2}-1114 \nu+301\right) p_{r}^{2}\right.\right. \\
& \left.+\left(400 \nu \delta-209 \delta-400 \nu^{2}+818 \nu-209\right) p^{2}+\left(355 \delta-704 \delta \nu+704 \nu^{2}-1414 \nu+355\right) \frac{1}{r}\right] \\
& \left.+\nu \chi_{1} \chi_{2}\left[(378-400 \nu) p^{2}+(512 \nu-1410) p_{r}^{2}+\frac{704 \nu-1182}{r}\right]+1 \leftrightarrow 2\right\} .
\end{aligned}
$$

\section{F. Factorizing the RR force into circular and noncircular parts}

The total RR force is the sum of the contributions calculated in the previous section, i.e.,

$$
\begin{aligned}
\mathcal{F}_{\phi} & =\mathcal{F}_{\phi}^{\mathrm{LO}}+\mathcal{F}_{\phi}^{1 \mathrm{PN}}+\mathcal{F}_{\phi}^{2 \mathrm{PN}}+\mathcal{F}_{\phi}^{\text {tail }}+\mathcal{F}_{\phi}^{\mathrm{SO}}+\mathcal{F}_{\phi}^{\mathrm{SS}}, \\
\mathcal{F}_{r} & =\mathcal{F}_{r}^{\mathrm{LO}}+\mathcal{F}_{r}^{1 \mathrm{PN}}+\mathcal{F}_{r}^{2 \mathrm{PN}}+\mathcal{F}_{r}^{\text {tail }}+\mathcal{F}_{r}^{\mathrm{SO}}+\mathcal{F}_{r}^{\mathrm{SS}} .
\end{aligned}
$$


We have checked that our gauge-dependent RR force agrees with that in Refs. $[78,92,93]$ by using the balance equations. Denoting the RR force from those references by $\left(\overline{\mathcal{F}}_{r}, \overline{\mathcal{F}}_{\phi}\right)$ with corresponding Schott terms $\left(\bar{E}_{\text {Schott }}, \bar{J}_{\text {Schott }}\right)$, Eqs. (7) lead to

$$
\begin{aligned}
\dot{\bar{r}} \overline{\mathcal{F}}_{r}+\dot{\bar{\phi}} \overline{\mathcal{F}}_{\phi}+\dot{\bar{E}}_{\text {Schott }} & =\dot{r} \mathcal{F}_{r}+\dot{\phi} \mathcal{F}_{\phi}+\dot{E}_{\text {Schott }}, \\
\overline{\mathcal{F}}_{\phi}+\dot{\bar{J}}_{\text {Schott }} & =\mathcal{F}_{\phi}+\dot{J}_{\text {Schott }} .
\end{aligned}
$$

Then, by writing an ansatz for $\left(\bar{E}_{\text {Schott }}, \bar{J}_{\text {Schott }}\right)$ with unknown coefficients, we checked that a solution exists, implying that $\left(\mathcal{F}_{r}, \mathcal{F}_{\phi}\right)$ and $\left(\overline{\mathcal{F}}_{r}, \overline{\mathcal{F}}_{\phi}\right)$ are related via a coordinate transformation.

To implement our results in the SEOBNRV4HM model, we factorize the RR force into a quasicircular part times eccentric corrections as in Eqs. (14) and (11), which read

$$
\begin{array}{ll}
\mathcal{F}_{\phi}=\mathcal{F}_{\phi}^{\mathrm{qc}} \mathcal{F}_{\phi}^{\mathrm{ecc}}, & \mathcal{F}_{r}=\mathcal{F}_{r}^{\mathrm{qc}} \mathcal{F}_{r}^{\mathrm{ecc}}, \\
\mathcal{F}_{\phi}^{\mathrm{qc}}=-\frac{\Phi_{E}^{\mathrm{qc}}}{\Omega}, & \mathcal{F}_{r}^{\mathrm{qc}}=-\frac{\Phi_{E}^{\mathrm{qc}} p_{r}}{\Omega p_{\phi}},
\end{array}
$$

and for the quasicircular part we use the unexpanded force used in SEOBNRV4HM, in which the energy flux has the following PN expansion in terms of the orbital velocity $v_{\Omega} \equiv \Omega^{1 / 3}$ :

$$
\begin{aligned}
\frac{\Phi_{E}^{\mathrm{qc}}}{\nu^{2}}= & \frac{32}{5} v_{\Omega}^{10}-\frac{2}{105 c^{2}}(980 \nu+1247) v_{\Omega}^{12}+\frac{128 \pi}{5 c^{3}} v_{\Omega}^{13} \\
& +\frac{4 v_{\Omega}^{13}}{5 c^{3}}\left[\chi_{1}(12 \nu-11 \delta-11)+\chi_{2}(12 \nu+11 \delta-11)\right] \\
& +\frac{2}{2835 c^{4}} v_{\Omega}^{14}\left(32760 \nu^{2}+166878 \nu-44711\right) \\
& +\frac{2}{5 c^{4}} v_{\Omega}^{14}\left[\left(32 C_{1 \mathrm{ES}^{2}}+1\right) \chi_{1}^{2} X_{1}^{2}+62 \nu \chi_{1} \chi_{2}\right. \\
& \left.+\left(32 C_{2 \mathrm{ES}^{2}}+1\right) \chi_{2}^{2} X_{2}^{2}\right] .
\end{aligned}
$$

This leads to the eccentric part

$$
\begin{aligned}
& \mathcal{F}_{\phi}^{\mathrm{ecc}}=\frac{29 p_{r}^{2} r-10 \dot{p}_{r} r^{2}+12}{12\left(\dot{p}_{r} r^{2}+1\right)^{2 / 3}}+\frac{[\cdots]}{c^{2} r\left(\dot{p}_{r} r^{2}+1\right)^{5 / 3}}+\cdots, \\
& \mathcal{F}_{r}^{\mathrm{ecc}}=\frac{7 p_{r}^{2} r-5 \dot{p}_{r} r^{2}+6}{6\left(\dot{p}_{r} r^{2}+1\right)^{2 / 3}}+\frac{[\cdots]}{c^{2} r\left(\dot{p}_{r} r^{2}+1\right)^{5 / 3}}+\cdots .
\end{aligned}
$$

The full 2PN expressions are provided in the Supplemental Material [88].

In these eccentric corrections to the RR force, we used $\dot{p}_{r}$ instead of $p_{\phi}$ because it improves the agreement of our model with SEOBNRV4HM in the quasicircular orbit limit, in which $p_{r}=0=\dot{p}_{r}$ leading to $\mathcal{F}_{r, \phi}^{\mathrm{ecc}}=1$. However, having $\dot{p}_{r}$ on the right-hand side of the EOM for $p_{r}$ would complicate solving the system of differential equations (4).
Therefore, when evolving the EOMs, we simply replace $\dot{p}_{r}$ in the RR force with the derivative of the Hamiltonian with respect to $r$ calculated numerically, i.e., $\dot{p}_{r} \rightarrow-\partial \hat{H}_{\mathrm{EOB}} / \partial r$.

SEOBNR waveform models use $p_{r_{*}}$ (the conjugate momentum to the tortoise radial coordinate $r_{*}$ ) instead of $p_{r}$ since it improves stability of the EOMs near the EOB event horizon $[69,112]$. The two momenta are related by Eq. (E3). In Appendix E, we also obtain Eq. (E8) for the transformation between $\dot{p}_{r}$ and $\dot{p}_{r_{*}}$.

\section{INITIAL CONDITIONS}

Having determined the RR force that enters the EOMs, we need to specify the initial conditions to be used in evolving the system of equations. In this section, we first review how the initial conditions are implemented in SEOBNRV4HM for quasicircular orbits $[46,94]$, and then discuss a simple extension for eccentric orbits.

\section{A. Initial conditions for quasicircular orbits}

Let us recapitulate the initial conditions for quasicircular/ spherical orbits in the SEOBNRV4HM model as derived in Refs. [46,94]. We start by specifying an initial orbital frequency $\Omega_{0}$, with initial orbital phase $\phi_{0}=0$, and solve

$$
\left[\frac{\partial H}{\partial r}\right]_{0}=0, \quad\left[\frac{\partial H}{\partial p_{\phi}}\right]_{0}=\Omega_{0}
$$

for the initial values of $r$ and $p_{\phi}$, while neglecting RR, $p_{r} \approx 0$. The initial condition for $p_{r}$ is then obtained by solving

$$
[\dot{r}]_{0}=\left[\frac{\partial H}{\partial p_{r}}\right]_{0}
$$

for $p_{r}$, after calculating $[\dot{r}]_{0}$ using the result from adiabatic evolution [46]

$$
[\dot{r}]_{0}=\left[\frac{d p_{\phi} / d t}{d p_{\phi} / d r}\right]_{0}=\left[\frac{\dot{E}}{d E / d r}\right]_{0}
$$

where $\dot{E}$ is the circular-orbits energy flux, and the derivative $d E / d r=d H / d r$ can be determined using the following equations for circular orbits:

$$
\begin{gathered}
d H=\frac{\partial H}{\partial r} d r+\frac{\partial H}{\partial p_{r}} d p_{r}+\frac{\partial H}{\partial p_{\phi}} d p_{\phi}, \\
p_{r}=0, \quad d p_{r}=0, \quad d\left(\frac{\partial H}{\partial r}\right)=0 .
\end{gathered}
$$

This leads to 


$$
\frac{d}{d r} \frac{\partial H}{\partial r}=0=\frac{\partial^{2} H}{\partial r^{2}}+\frac{\partial^{2} H}{\partial r \partial p_{\phi}} \frac{d p_{\phi}}{d r},
$$

which can be solved for $d p_{\phi} / d r$ to obtain

$$
\frac{d p_{\phi}}{d r}=-\frac{\partial^{2} H / \partial r^{2}}{\partial^{2} H / \partial r \partial p_{\phi}} .
$$

Plugging that solution into $d H / d r=\left(\partial H / \partial p_{\phi}\right) d p_{\phi} / d r$ yields the result in Eq. (4.14) of Ref. [94], which reads

$$
\frac{d H}{d r}=-\frac{\left(\partial H / \partial p_{\phi}\right)\left(\partial^{2} H / \partial r^{2}\right)}{\partial^{2} H / \partial r \partial p_{\phi}},
$$

and hence

$$
\left[\frac{\partial H}{\partial p_{r}}\right]_{0}=[\dot{r}]_{0}=\left[-\frac{d E}{d t} \frac{\partial^{2} H / \partial r \partial p_{\phi}}{\left(\partial H / \partial p_{\phi}\right)\left(\partial^{2} H / \partial r^{2}\right)}\right]_{0} .
$$

The complete procedure to obtain the initial conditions for the orbital phase space is now as follows. Given $\Omega_{0}$, masses, and spins, we numerically solve the relations in Eq. (60) for the initial values $r_{0}$ and $p_{\phi_{0}}$, choosing $\phi_{0}=0$ and assuming $p_{r} \approx 0$. Using these values, we numerically solve Eq. (68) for the initial value $p_{r 0}$.

\section{B. Initial conditions for eccentric orbits}

Since eccentricity is a gauge-dependent concept, we do not need to calculate accurate initial conditions for eccentric orbits in a specific gauge. Instead, we can choose a measure for eccentricity that can be adjusted to be as convenient as possible for numerical implementation. The only strict requirement is that for zero eccentricity $e=0$ one recovers the quasicircular case. Hence, we can start with very accurate initial conditions for quasicircular orbits and perturb them for eccentric orbits.

We choose to specify an initial orbital frequency $\Omega_{0}$ and an initial eccentricity $e_{0}$ using the Keplerian parametrization $1 / r=u_{p}(1+e \cos \chi)$. We also assume that the orbit starts with $\phi_{0}=0$ at periastron $(\chi=0)$, where $p_{r}=0$ in absence of RR, which simplifies calculating the initial conditions for $r$ and $p_{\phi}$. An advantage of starting at periastron instead of apastron is that the specified initial frequency is then the maximum orbital frequency (over the first orbit), and can be used to estimate the frequency at which the binary enters a GW detector's frequency band.

To obtain $r_{0}$ and $p_{\phi_{0}}$, we solve Eq. (60) with a nonzero $\dot{p}_{r}$, i.e.,

$$
\left[\frac{\partial H}{\partial r}\right]_{0}=-\left[\dot{p}_{r}\left(p_{\phi}, e\right)\right]_{0}, \quad\left[\frac{\partial H}{\partial p_{\phi}}\right]_{0}=\Omega_{0},
$$

with $p_{r} \approx 0$, and $\left[\dot{p}_{r}\right]_{0}$ given as a $2 \mathrm{PN}$ expansion in terms of $p_{\phi}$ and $e$. For quasicircular orbits, these equations reduce exactly to Eqs. (60) since $\dot{p}_{r} \propto e$.

To obtain the PN expansion for $\dot{p}_{r}$ at periastron, we first invert the Hamiltonian at the turning points $r_{ \pm}=$ $1 /\left(u_{p}(1 \pm e)\right)$ with $p_{r}=0$ and solve for the energy and angular momentum as functions of $e$ and $u_{p}$, which are given by Eqs. (D2). Then, we invert $p_{\phi}\left(e, u_{p}\right)$ to obtain Eq. (D3) for $u_{p}\left(p_{\phi}, e\right)$ and insert it into the PN expansion for $\dot{p}_{r}=-\partial H / \partial r$ at periastron $\left(r=1 /\left[u_{p}(1+e)\right]\right)$. This yields

$$
\begin{aligned}
{\left[\dot{p}_{r}\right]_{0}=} & \frac{e_{0}\left(e_{0}+1\right)^{2}}{p_{\phi 0}^{4}}+\frac{e_{0}\left(e_{0}+1\right)^{2}}{2 c^{2} p_{\phi 0}^{6}}\left[e_{0}^{2}(5-\nu)-4 e_{0}+\nu+7\right] \\
& +\frac{e_{0}\left(e_{0}+1\right)^{2}\left(3 e_{0}^{2}-2 e_{0}+3\right)}{2 c^{3} p_{\phi 0}^{7}}\left[(\nu-2 \delta-2) \chi_{1}+(\nu+2 \delta-2) \chi_{2}\right] \\
& +\frac{e_{0}\left(e_{0}+1\right)^{2}}{8 c^{4} p_{\phi 0}^{8}}\left[3 \nu^{2}-\nu+95-6 e_{0}^{2}\left(\nu^{2}+5 \nu-39\right)+8 e_{0}(\nu-25)+e_{0}^{4}\left(3 \nu^{2}-17 \nu+55\right)+8 e_{0}^{3}(\nu-7)\right] \\
& +\frac{e_{0}\left(e_{0}+1\right)^{2}}{2 c^{4} p_{\phi 0}^{8}}\left\{\chi_{1}^{2}\left[C_{1 \mathrm{ES}^{2}}\left(3 e_{0}^{2}-2 e_{0}+3\right) X_{1}^{2}+\left(5 e_{0}^{2}-6 e_{0}-3\right) X_{1}^{4}\right]\right. \\
& \left.+\nu \chi_{1} \chi_{2}\left[e_{0}^{2}(5 \nu+3)-2 e_{0}(3 \nu+1)-3 \nu+3\right]+1 \leftrightarrow 2\right\} .
\end{aligned}
$$

The initial condition can now be obtained in analogy to the quasicircular case: given $\Omega_{0}, e_{0}$, masses, and spins, we obtain $r_{0}$ and $p_{\phi_{0}}$ from Eqs. (69) and (70) (assuming $\left.p_{r} \approx 0\right), p_{r 0}$ then follows from Eq. (68) as before, and $\phi_{0}=0$ by convention. We can keep using the circularorbits energy flux in Eq. (68), instead of replacing $\dot{E}$ with
$\Omega \mathcal{F}_{\phi}$ for eccentric orbits, because the difference on the orbital dynamics is negligible since it involves $p_{r}$ (which at periastron is numerically much smaller than $r$ or $p_{\phi}$ ).

To assess the accuracy of the initial conditions for eccentric orbits, we compare the specified value for the eccentricity in the Keplerian parametrization with the value 
TABLE I. Nonspinning initial conditions given the parameters $\left(e_{0}, \Omega_{0}, \nu\right)$ and the eccentricity $e_{\Omega}$ measured from the orbital frequency using Eq. (71). The initial frequency $\Omega_{0}$ was chosen to give $\sim 30 \mathrm{GW}$ cycles between $r_{0}$ and $r=5$.

\begin{tabular}{lllrrrl}
\hline \hline$e_{0}$ & $\Omega_{0}$ & \multicolumn{1}{c}{$\nu$} & \multicolumn{1}{c}{$r_{0}$} & $p_{\phi_{0}}$ & $p_{r 0}$ & \multicolumn{1}{c}{$e_{\Omega}$} \\
\hline 0.01 & 0.03 & 0.25 & 10.4 & 3.8 & -0.0012 & 0.0087 \\
0.2 & 0.045 & 0.25 & 8.1 & 3.7 & -0.0024 & 0.19 \\
0.7 & 0.065 & 0.25 & 6.7 & 4.0 & -0.0033 & 0.69 \\
0.2 & 0.058 & 0.1 & 6.8 & 3.6 & -0.0011 & 0.20 \\
\hline \hline
\end{tabular}

calculated from the orbital frequency [or the frequency of the $(2,2)$ mode] at periastron $\Omega_{p}$ and apastron $\Omega_{a}$, which is given by [113]

$$
e_{\Omega}=\frac{\sqrt{\Omega_{p}}-\sqrt{\Omega_{a}}}{\sqrt{\Omega_{p}}+\sqrt{\Omega_{a}}},
$$

and to calculate it, we follow the steps explained after Eq. (2.8) of Ref. [41]. Since we evaluate $e_{\Omega}$ by evolving the binary over one orbit (including RR), it only holds approximately that $\Omega_{p} \approx \Omega_{a}$ in the quasicircular case. That is, $e_{\Omega}$ does not vanish exactly for quasicircular orbits, in contrast to our $e_{0}$. Table I shows the value of the eccentricity $e_{\Omega}$ calculated from the orbital frequency compared to the specified eccentricity $e_{0}$, and we see good agreement between the two measures of eccentricity.

\section{GRAVITATIONAL WAVEFORM MODES}

In this section, we obtain the $2 \mathrm{PN}$ waveform modes to 2PN order including LO tail effects, and SO and SS couplings for aligned spins. The instantaneous nonspinning part of the modes was derived for eccentric orbits in Refs. [114,115] in harmonic coordinates and we convert their results to EOB coordinates. For the LO tail part, we extend the results of Ref. [79] to $\mathcal{O}\left(e^{6}\right)$ and to higher modes using the Keplerian parametrization, and then we convert those results to an expansion in $p_{r}$ and $\dot{p}_{r}$. The spin contributions to the modes were derived to $2 \mathrm{PN}$ order for circular orbits in Ref. [116], and here we derive them for eccentric orbits.

The GW spherical harmonic modes $h^{\ell m}$ are the expansion of the complex polarization waveform $h=h_{+}-i h_{\times}$ into spin-weighted $s=-2$ spherical harmonics $Y_{-2}^{\ell m}(\Theta, \Phi)$ such that

$$
h_{+}-i h_{\times}=\sum_{\ell=2}^{\infty} \sum_{m=-\ell}^{\ell} h^{\ell m} Y_{-2}^{\ell m}(\Theta, \Phi) .
$$

The modes $h^{\ell m}$ can be calculated directly from the radiative multipole moments via [117-119]

$$
h^{\ell m}=\frac{1}{\sqrt{2} D_{L} c^{\ell+2}}\left[U^{\ell m}-\frac{i}{c} V^{\ell m}\right],
$$

where $D_{L}$ is the luminosity distance of the source and the radiative multipole moments are related to the symmetric trace-free (STF) moments $U_{L}$ and $V_{L}$ by

$$
\begin{aligned}
U^{\ell m} & =\frac{16 \pi}{(2 \ell+1) ! !} \sqrt{\frac{(\ell+1)(\ell+2)}{2 \ell(\ell-1)}} \overline{\mathcal{Y}}_{L}^{\ell m} U_{L}, \\
V^{\ell m} & =\frac{-32 \pi}{(2 \ell+1) ! !} \sqrt{\frac{\ell(\ell+2)}{2(\ell+1)(\ell-1)}} \overline{\mathcal{Y}}_{L}^{\ell m} V_{L},
\end{aligned}
$$

where $\overline{\mathcal{Y}}_{L}^{\ell m}$ is the complex conjugate of the STF tensors relating the unit vectors $N_{\langle L\rangle}$ (which point from the source to the detector) to the spherical harmonics basis $Y^{\ell m}(\Theta, \Phi)$ such that

$$
\begin{gathered}
Y^{\ell m}(\Theta, \Phi)=\mathcal{Y}_{L}^{\ell m} N_{\langle L\rangle}(\Theta, \Phi), \\
N_{\langle L\rangle}(\Theta, \Phi)=\frac{4 \pi \ell !}{(2 \ell+1) ! !} \sum_{m=-\ell}^{\ell} \overline{\mathcal{Y}}_{L}^{\ell m} Y^{\ell m}(\Theta, \Phi), \\
\overline{\mathcal{Y}}_{L}^{\ell m}=\frac{(2 \ell+1) ! !}{4 \pi \ell !} \int d \Omega N_{\langle L\rangle} \bar{Y}^{\ell m}, \\
\overline{\mathcal{Y}}_{L}^{\ell m} \mathcal{Y}_{L}^{\ell m^{\prime}}=\frac{(2 \ell+1) ! !}{4 \pi \ell !} \delta_{m m^{\prime}},
\end{gathered}
$$

and we can express the unit vector $\boldsymbol{N}$ in terms of the angles $\Theta$ and $\Phi$ as

$$
\boldsymbol{N}=\sin \Theta \cos \Phi \hat{\boldsymbol{e}}_{x}+\sin \Theta \sin \Phi \hat{\boldsymbol{e}}_{y}+\cos \Theta \hat{\boldsymbol{e}}_{z} .
$$

For planar binaries, nonspinning or with aligned spins, it was shown in Ref. [119] that the modes can be determined using the mass-type multipole moments for even $\ell+m$, or the current-type multipole moments for odd $\ell+m$, i.e.,

$$
\begin{aligned}
h^{\ell m} & =\frac{1}{\sqrt{2} D_{L} c^{\ell+2}} U^{\ell m}, & \ell+m \text { even }, \\
h^{\ell m} & =-\frac{i}{\sqrt{2} D_{L} c^{\ell+3}} V^{\ell m}, & \ell+m \text { odd } .
\end{aligned}
$$

We define $H^{\ell m}$ such that

$$
h^{\ell m}=-\frac{8 \mu}{c^{4} D_{L}} \sqrt{\frac{\pi}{5}} \mathrm{e}^{-i m \phi} H^{\ell m},
$$

which makes the LO part of $H^{22}=x$ for circular orbits. Note that different conventions for the phase origin contribute a factor of $(-i)^{m}$ to the modes [120].

In this paper, we compute the modes to $2 \mathrm{PN}$ order beyond the leading order of the $(2,2)$ mode, which means we consider modes up to the $\ell=6, m=$ even modes. To $2 \mathrm{PN}$ order, the instantaneous contributions to the radiative 
multipole moments coincide with the source multipole moments. Including the hereditary terms that contribute to $2 \mathrm{PN}$, the radiative multipole moments are given by $[115,117,118]$

$$
\begin{aligned}
U_{i j} & =I_{i j}^{(2)}+\frac{2 M}{c^{3}} \int_{0}^{\infty} d \tau I_{i j}^{(4)}(t-\tau) \ln \left(\frac{\tau}{b_{1}}\right)+\mathcal{O}\left(\frac{1}{c^{5}}\right), \\
U_{i j k} & =I_{i j k}^{(3)}+\frac{2 M}{c^{3}} \int_{0}^{\infty} d \tau I_{i j k}^{(5)}(t-\tau) \ln \left(\frac{\tau}{b_{2}}\right)+\mathcal{O}\left(\frac{1}{c^{5}}\right), \\
U_{L} & =I_{L}^{(\ell)}+\mathcal{O}\left(\frac{1}{c^{3}}\right), \\
V_{i j} & =J_{i j}^{(2)}+\frac{2 M}{c^{3}} \int_{0}^{\infty} d \tau J_{i j}^{(4)}(t-\tau) \ln \left(\frac{\tau}{b_{3}}\right)+\mathcal{O}\left(\frac{1}{c^{5}}\right), \\
V_{L} & =J_{L}^{(\ell)}+\mathcal{O}\left(\frac{1}{c^{3}}\right),
\end{aligned}
$$

where the constants $b_{i}$ are gauge parameters that will be eliminated via a phase shift as was done in Ref. [117]. The source multipole moments for nonspinning binaries are given in, e.g., Refs. [115,117], while the spin contributions to the source moments are given in Refs. $[116,121]$.

\section{A. Instantaneous nonspinning contributions}

The instantaneous contributions to the modes for nonspinning binaries in eccentric orbits were derived in Ref. [114] to 2PN and in Ref. [115] to 3PN. The results of Ref. [115] are in harmonic coordinates and in terms of the variables $(r, \phi, \dot{r}, \dot{\phi})$. Hence, we can simply transform their results from harmonic to EOB coordinates using the transformations in Appendix A. For the $(2,2)$ mode we obtain

$$
\begin{aligned}
\hat{H}_{\text {inst }}^{22}= & \frac{1}{2}\left(\frac{1}{r}+p^{2}-2 p_{r}^{2}\right)+i \frac{p_{\phi} p_{r}}{r}+\frac{1}{c^{2}}\left\{\left(\frac{\nu}{28}-\frac{5}{28}\right) p^{4}+\left(\frac{31 \nu}{28}-\frac{157}{84}\right) \frac{p^{2}}{r}+\left(\frac{5}{14}-\frac{\nu}{14}\right) p_{r}^{2} p^{2}+\left(\frac{13}{3}-\nu\right) \frac{p_{r}^{2}}{r}\right. \\
& \left.+\left(\frac{\nu}{2}-2\right) \frac{1}{r^{2}}+i \frac{p_{\phi} p_{r}}{r}\left[\left(\frac{\nu}{14}-\frac{5}{14}\right) p^{2}+\left(\frac{2 \nu}{7}-\frac{185}{42}\right) \frac{1}{r}\right]\right\}+\frac{1}{c^{4}}\left\{\left(-\frac{17 \nu^{2}}{336}-\frac{13 \nu}{336}+\frac{5}{48}\right) p^{6}\right. \\
& +\left(-\frac{671 \nu^{2}}{1008}-\frac{1375 \nu}{1008}+\frac{481}{504}\right) \frac{p^{4}}{r}+\left(\frac{127 \nu^{2}}{54}-\frac{1355 \nu}{189}-\frac{5519}{3024}\right) \frac{p^{2}}{r^{2}}+\left(\frac{17 \nu^{2}}{168}+\frac{13 \nu}{168}-\frac{5}{24}\right) p_{r}^{2} p^{4} \\
& +\left(-\frac{67 \nu^{2}}{126}+\frac{20 \nu}{9}-\frac{659}{504}\right) \frac{p_{r}^{2} p^{2}}{r}+\left(-\frac{464 \nu^{2}}{189}+\frac{2249 \nu}{756}-\frac{811}{3024}\right) \frac{p_{r}^{2}}{r^{2}}+\left(\frac{17 \nu^{2}}{18}-\frac{25 \nu}{36}-\frac{919}{1008}\right) \frac{p_{r}^{4}}{r} \\
& +\left(\frac{205 \nu^{2}}{252}-\frac{49 \nu}{36}+\frac{95}{63}\right) \frac{1}{r^{3}}+i \frac{p_{r} p_{\phi}}{r}\left[\left(-\frac{17 \nu^{2}}{168}-\frac{13 \nu}{168}+\frac{5}{24}\right) \frac{p^{4}}{r}+\left(-\frac{4 \nu^{2}}{21}+\frac{29 \nu}{28}+\frac{67}{56}\right) \frac{p^{2}}{r^{2}}\right. \\
& \left.\left.+\left(-\frac{523 \nu^{2}}{378}+\frac{1226 \nu}{189}+\frac{193}{54}\right) \frac{1}{r^{3}}+\left(\frac{43 \nu^{2}}{63}-\frac{125 \nu}{126}+\frac{787}{504}\right) \frac{p_{r}^{2}}{r^{2}}\right]\right\} .
\end{aligned}
$$

The expressions for the other modes that contribute to $2 \mathrm{PN}$, i.e., up to $\ell=|m|=6$, are provided as a Mathematica file in the Supplemental Material [88]. Note that the $(\ell, 0)$ modes are zero for circular orbits but not for eccentric orbits. For example, the LO part of the $(2,0)$ mode is given by

$$
\hat{H}_{\mathrm{inst}}^{20}=\frac{1}{\sqrt{6}}\left(p^{2}-\frac{1}{r}\right)+\mathcal{O}\left(\frac{1}{c^{2}}\right)
$$

which is zero for circular orbits since $p^{2}=1 / r+\cdots$.

\section{B. Hereditary contributions}

The hereditary contributions to the modes can be calculated analytically in an eccentricity expansion, as was done in Ref. [79] for the $(2,2)$ mode to $\mathcal{O}\left(e^{2}\right)$, and in Ref. [122] for all modes to $3 \mathrm{PN}$ order and to $\mathcal{O}\left(e^{6}\right)$. The results of Ref. [122] use the quasi-Keplerian parametrization, while here we use the Keplerian parametrization following the method developed in Ref. [79], which is based on results from Refs. [96,117,123], to derive the leading order tail effects that contribute to the modes up to $2 \mathrm{PN}$ order and to $\mathcal{O}\left(e^{6}\right)$. (See Ref. [79] for a discussion of the advantages of the Keplerian parametrization over the quasi-Keplerian parametrization.) We finally convert the eccentricityexpanded tail contributions to an expansion in $p_{r}$ and $\dot{p}_{r}$.

\section{Modes with even $\ell+m$}

The LO mass-type multipole moments are given by [96]

$$
I^{L}=\mu s_{\ell} r^{\ell} n^{\langle L\rangle}
$$

where $s_{\ell} \equiv X_{2}^{\ell-1}+(-1)^{\ell} X_{1}^{\ell-1}$, and the unit vectors $n^{\langle L\rangle}$ are related to spherical harmonics via Eq. (75), leading to 


$$
I^{L}=\sum_{m=-\ell}^{\ell} \mathcal{Y}_{\ell m}^{L} a_{\ell m} r^{\ell} \mathrm{e}^{-i m \phi}
$$

with the coefficients (for equatorial orbits)

$$
a_{\ell m} \equiv \frac{4 \pi \mu s_{\ell} \ell !}{(2 \ell+1) ! !} \bar{Y}_{\ell m}\left(\frac{\pi}{2}, 0\right)
$$

Decomposing the phase $\phi$ into an oscillatory part and a linearly growing part $\phi=\phi_{0}+\omega_{\phi} t+\Delta \phi_{r}$ allows expressing the oscillatory part $\Delta \phi_{r}$ as a Fourier series expansion. Hence,

$$
I^{L}=\sum_{m=-\ell}^{\ell} \mathcal{Y}_{\ell m}^{L} a_{\ell m} J_{\ell m} \mathrm{e}^{-i m \psi_{\phi}},
$$

with the functions $J_{\ell m}$ defined by

$$
J_{\ell m}=r^{\ell} \mathrm{e}^{-i m \phi_{0}} \mathrm{e}^{-i m \Delta \phi_{r}}=\sum_{k=-\infty}^{\infty} J_{\ell m k} \mathrm{e}^{-i k \psi_{r}},
$$

where $\psi_{r}$ and $\psi_{\phi}$ are the radial and azimuthal angle variables associated with the frequencies $\omega_{r}=d \psi_{r} / d t$ and $\omega_{\phi}=d \psi_{\phi} / d t$. The coefficients $J_{\ell m k}$ are given by

$$
\begin{aligned}
J_{\ell m k} & =\frac{1}{2 \pi} \int_{0}^{2 \pi} d \psi_{r} \mathrm{e}^{i k \psi_{r}} J_{\ell m} \\
& =\frac{\omega_{r}}{2 \pi} \int_{0}^{2 \pi} \frac{d \chi}{\mathcal{P}} r^{\ell} \mathrm{e}^{-i m \phi_{0}} \mathrm{e}^{-i m \Delta \phi_{r}} \mathrm{e}^{i k \psi_{r}} \\
& =\frac{\omega_{r}}{2 \pi} u_{p}^{-\ell-3 / 2} \int_{0}^{2 \pi} \frac{d \chi}{(1+e \cos \chi)^{\ell+2}} \mathrm{e}^{-i m \Delta \phi_{r}} \mathrm{e}^{i k \psi_{r}},
\end{aligned}
$$

where, in the last line, we assume $\phi_{0}=0$. The function $\mathcal{P}$ denotes the conservative part of $\dot{\chi}$ and is related to the radial angle $\psi_{r}$ via $d \psi_{r} / d \chi=\omega_{r} / \mathcal{P}$, with $\mathcal{P}=(1+e \cos \chi)^{2} u_{p}^{3 / 2}$ at LO (see Ref. [79] for more details).

Thus, the Newtonian mass multipole moments can be expressed as

$$
I^{L}=\sum_{m=-\ell}^{\ell} \sum_{k=-\infty}^{\infty} \mathcal{Y}_{\ell m}^{L} a_{\ell m} J_{\ell m k} \mathrm{e}^{-i\left(k \psi_{r}+m \psi_{\phi}\right)},
$$

where the azimuthal angle is related to the radial angle by $\psi_{\phi}=\phi-\Delta \phi_{r}$ with $\Delta \phi_{r}=\chi-\psi_{r}$ at LO. This allows us to write the LO tail contribution to the mass-type radiative moments as

$$
\begin{aligned}
U_{L}^{\text {tail }}= & \frac{2 M}{c^{3}} \int_{0}^{\infty} d \tau I_{L}^{(\ell+2)}(t-\tau) \ln \left(\frac{\tau}{b}\right) \\
= & (-i)^{\ell+2} \frac{2 M}{c^{3}} \sum_{m=-\ell}^{\ell} \sum_{k=-\infty}^{\infty} \mathcal{Y}_{L}^{\ell m} a_{\ell m} J_{\ell m k} \Omega_{m k}^{\ell+2} \\
& \times \mathrm{e}^{-i\left(k \psi_{r}+m \psi_{\phi}\right)} \mathcal{I}\left(\Omega_{m k}\right),
\end{aligned}
$$

where $\Omega_{m k} \equiv m \omega_{\phi}+k \omega_{r}$ and

$$
\begin{aligned}
\mathcal{I}(x) & \equiv \int_{0}^{\infty} d \tau \mathrm{e}^{i x \tau} \ln \left(\frac{\tau}{b}\right) \\
& =-\frac{1}{x}\left[\frac{\pi}{2} \operatorname{sgn}(x)+i \ln (|x| b)+i \gamma_{E}\right] .
\end{aligned}
$$

The exponential $\mathrm{e}^{-i k \psi_{r}}$ can be expressed in terms of $\chi$ and $e$ by integrating $d \psi_{r}=\omega_{r} d \chi / \mathcal{P}$, with $\omega_{r}=\left(u_{p}-e^{2} u_{p}\right)^{3 / 2}$, leading to Eq. (3.41) of Ref. [79], which reads

$$
\mathrm{e}^{-i k \psi_{r}}=\mathrm{e}^{i k \frac{e \sqrt{1-e^{2}} \sin \chi}{1+e \cos \chi}}\left(\frac{1+\sqrt{1-e^{2}}+e \mathrm{e}^{i \chi}}{e+\left(1+\sqrt{1-e^{2}}\right) \mathrm{e}^{i \chi}}\right)^{k} .
$$

Hence, the modes with $\ell=2$ and $m$ even are given by

$$
\begin{aligned}
h_{\text {tail }}^{2 m}= & \frac{\sqrt{24} M a_{2 m}}{c^{7} R} \mathrm{e}^{-i m \phi} \sum_{k=-\infty}^{\infty} J_{2 m k} \Omega_{m k}^{4} \\
& \times \mathrm{e}^{i m \chi} \mathrm{e}^{-i(k+m) \psi_{r}} \mathcal{I}\left(\Omega_{m k}\right),
\end{aligned}
$$

while the modes with $\ell=3$ and $m$ odd are given by

$$
\begin{aligned}
h_{\text {tail }}^{3 m}= & -i \frac{4 \sqrt{5} M a_{3 m}}{3 \sqrt{6} c^{8} R} \mathrm{e}^{-i m \phi} \sum_{k=-\infty}^{\infty} J_{3 m k} \Omega_{m k}^{5} \\
& \times \mathrm{e}^{i m \chi} \mathrm{e}^{-i(k+m) \psi_{r}} \mathcal{I}\left(\Omega_{m k}\right) .
\end{aligned}
$$

To obtain analytical expressions for the modes, we expand the above equations in eccentricity, where the infinite sum over $k$ can be stopped at the order of the expansion in $e$. The result of that expansion is complicated, but we can perform a phase redefinition in the leading order instantaneous part ${ }^{3}$ of the form $\phi \rightarrow \phi+x^{3 / 2} \delta_{\phi}$ and absorb in $\delta_{\phi}$ all terms that are not proportional to $\pi^{3 / 2}$. This modifies the phase at $4 \mathrm{PN}$ relative order, which we can ignore when working to $2 \mathrm{PN}$ order. (See Ref. [117] for more details.) The result for the $(2,2)$ mode to $\mathcal{O}\left(e^{6}\right)$ is given by

\footnotetext{
${ }^{3}$ One first needs to express the leading order part in terms of the variables $(e, x, \chi)$ instead of $\left(r, p_{r}, p_{\phi}\right)$ using the relations from Appendix D. For example, for the $(2,2)$ mode, we obtain

$$
h_{\mathrm{LO}}^{22}=\frac{-8 \mu}{c^{4} D_{L}} \sqrt{\frac{\pi}{5}} \mathrm{e}^{-2 i \phi} \frac{x}{1-e^{2}}\left[1+\frac{e}{4}\left(\mathrm{e}^{-i \chi}+5 \mathrm{e}^{i \chi}\right)+\frac{e^{2}}{2} \mathrm{e}^{2 i \chi}\right] .
$$
}




$$
\begin{aligned}
\hat{H}_{\text {tail }}^{22}= & \frac{2 \pi}{c^{3}} x^{5 / 2}\left[1+e\left(\frac{11 \mathrm{e}^{-i \chi}}{8}+\frac{13 \mathrm{e}^{i \chi}}{8}\right)+e^{2}\left(\frac{5}{8} \mathrm{e}^{-2 i \chi}+\frac{7}{8} \mathrm{e}^{2 i \chi}+4\right)+e^{3}\left(\frac{121 \mathrm{e}^{-i \chi}}{32}+\frac{143 \mathrm{e}^{i \chi}}{32}+\frac{3}{32} \mathrm{e}^{-3 i \chi}+\frac{1}{12} \mathrm{e}^{3 i \chi}\right)\right. \\
& +e^{4}\left(\frac{25}{16} \mathrm{e}^{-2 i \chi}+\frac{203}{96} \mathrm{e}^{2 i \chi}-\frac{5}{96} \mathrm{e}^{4 i \chi}+\frac{65}{8}\right)+e^{5}\left(\frac{55 \mathrm{e}^{-i \chi}}{8}+\frac{6233 \mathrm{e}^{i \chi}}{768}+\frac{15}{64} \mathrm{e}^{-3 i \chi}+\frac{281 \mathrm{e}^{3 i \chi}}{1536}+\frac{53 \mathrm{e}^{5 i \chi}}{7680}\right) \\
& \left.+e^{6}\left(\frac{175}{64} \mathrm{e}^{-2 i \chi}+\frac{1869}{512} \mathrm{e}^{2 i \chi}-\frac{449 \mathrm{e}^{4 i \chi}}{3840}+\frac{31 \mathrm{e}^{6 i \chi}}{23040}+\frac{30247}{2304}\right)\right],
\end{aligned}
$$

while for the $(2,0)$ mode

$$
\begin{aligned}
\hat{H}_{\text {tail }}^{20}= & \frac{\pi x^{5 / 2}}{2 \sqrt{6} c^{3}}\left[e\left(\mathrm{e}^{-i \chi}+\mathrm{e}^{i \chi}\right)+e^{2}\left(\mathrm{e}^{-2 i \chi}+\mathrm{e}^{2 i \chi}+2\right)+e^{3}\left(3 \mathrm{e}^{-i \chi}+3 \mathrm{e}^{i \chi}+\frac{1}{4} \mathrm{e}^{-3 i \chi}+\frac{1}{4} \mathrm{e}^{3 i \chi}\right)+e^{4}\left(\frac{29}{12} \mathrm{e}^{-2 i \chi}+\frac{29}{12} \mathrm{e}^{2 i \chi}+\frac{9}{2}\right)\right. \\
& \left.+e^{5}\left(\frac{179 \mathrm{e}^{-i \chi}}{32}+\frac{179 \mathrm{e}^{i \chi}}{32}+\frac{125}{192} \mathrm{e}^{-3 i \chi}+\frac{125}{192} \mathrm{e}^{3 i \chi}\right)+e^{6}\left(\frac{805}{192} \mathrm{e}^{-2 i \chi}+\frac{805}{192} \mathrm{e}^{2 i \chi}-\frac{7}{960} \mathrm{e}^{-4 i \chi}-\frac{7}{960} \mathrm{e}^{4 i \chi}+\frac{121}{16}\right)\right] .
\end{aligned}
$$

The $(3,3)$ mode is given by

$$
\begin{aligned}
\hat{H}_{\text {tail }}^{33}= & -\frac{9 i \pi \delta}{4 c^{4}} \sqrt{\frac{15}{14}} x^{3}\left[1+e\left(\frac{47 \mathrm{e}^{-i \chi}}{27}+\frac{19 \mathrm{e}^{i \chi}}{9}\right)+e^{2}\left(\frac{61}{54} \mathrm{e}^{-2 i \chi}+\frac{91}{54} \mathrm{e}^{2 i \chi}+\frac{155}{27}\right)+e^{3}\left(\frac{691 \mathrm{e}^{-i \chi}}{108}+\frac{841 \mathrm{e}^{i \chi}}{108}+\frac{35 \mathrm{e}^{-3 i \chi}}{108}+\frac{65 \mathrm{e}^{3 i \chi}}{108}\right)\right. \\
& +e^{4}\left(\frac{32}{9} \mathrm{e}^{-2 i \chi}+\frac{287}{54} \mathrm{e}^{2 i \chi}+\frac{5 \mathrm{e}^{-4 i \chi}}{144}+\frac{115 \mathrm{e}^{4 i \chi}}{1728}+\frac{3139}{216}\right)+e^{5}\left(\frac{503 \mathrm{e}^{-i \chi}}{36}+\frac{613 \mathrm{e}^{i \chi}}{36}+\frac{35 \mathrm{e}^{-3 i \chi}}{36}+\frac{3095 \mathrm{e}^{3 i \chi}}{1728}-\frac{457 \mathrm{e}^{5 i \chi}}{25920}\right) \\
& \left.+e^{6}\left(\frac{131}{18} \mathrm{e}^{-2 i \chi}+\frac{150503 \mathrm{e}^{2 i \chi}}{13824}+\frac{5}{48} \mathrm{e}^{-4 i \chi}+\frac{151}{810} \mathrm{e}^{4 i \chi}-\frac{41 \mathrm{e}^{6 i \chi}}{20736}+\frac{219}{8}\right)\right],
\end{aligned}
$$

and the $(3,1)$ mode

$$
\begin{aligned}
\hat{H}_{\text {tail }}^{31}= & \frac{i \pi \delta x^{3}}{12 \sqrt{14} c^{4}}\left[1+e\left(\mathrm{e}^{i \chi}-9 \mathrm{e}^{-i \chi}\right)+e^{2}\left(-\frac{27}{2} \mathrm{e}^{-2 i \chi}-\frac{5}{4} \mathrm{e}^{2 i \chi}-15\right)+e^{3}\left(-\frac{177}{4} \mathrm{e}^{-i \chi}-\frac{19 \mathrm{e}^{i \chi}}{2}-\frac{25}{4} \mathrm{e}^{-3 i \chi}-\frac{4}{3} e^{3 i \chi}\right)\right. \\
& +e^{4}\left(-\frac{89}{2} \mathrm{e}^{-2 i \chi}-\frac{125}{24} \mathrm{e}^{2 i \chi}-\frac{15}{16} \mathrm{e}^{-4 i \chi}-\frac{55 \mathrm{e}^{4 i x}}{192}-\frac{1703}{32}\right)+e^{5}\left(-\frac{10141}{96} \mathrm{e}^{-i \chi}-\frac{2867 \mathrm{e}^{i \chi}}{96}-\frac{75}{4} \mathrm{e}^{-3 i \chi}-\frac{629}{192} \mathrm{e}^{3 i \chi}-\frac{7}{960} \mathrm{e}^{5 i \chi}\right) \\
& \left.+e^{6}\left(-\frac{142903 \mathrm{e}^{-2 i \chi}}{1536}-\frac{2965}{256} \mathrm{e}^{2 i \chi}-\frac{45}{16} \mathrm{e}^{-4 i x}-\frac{239}{240} \mathrm{e}^{4 i \chi}+\frac{37 \mathrm{e}^{6 i \chi}}{23040}-\frac{16343}{144}\right)\right]
\end{aligned}
$$

We checked that our results agree with those of Ref. [122] after converting between the quasi-Keplerian and Keplerian parametrization, and performing a phase shift.

To express the modes in terms of $\left(r, p_{r}, p_{\phi}\right)$ instead of $(x, e, \chi)$, we use the following leading order relations:

$$
p_{\phi}=\frac{1}{\sqrt{u_{p}}}, \quad p_{r}=e \sqrt{u_{p}} \sin \chi, \quad \frac{1}{r}=u_{p}(1+e \cos \chi), \quad x=u_{p}\left(1-e^{2}\right)
$$

As explained above, it is advantageous to replace $p_{\phi}^{2}$ with $\dot{p}_{r}$ using $\dot{p}_{r}=\left(p_{\phi}^{2}-r\right) / r^{3}$ and expand in both $p_{r}$ and $\dot{p}_{r}$ (since $p_{r}$ and $\dot{p}_{r}$ are both of order $e$ ) to obtain 


$$
\begin{aligned}
& \hat{H}_{\text {tail }}^{22}=\frac{2 \pi}{c^{3}}\left\{\frac{p_{\phi}}{r^{3}}+\frac{i p_{r}}{4 r^{2}}+\left[\frac{7}{32} p_{\phi} p_{r}^{2} \dot{p}_{r}-\frac{7}{96} p_{\phi} r^{3} \dot{p}_{r}^{3}+i\left(\frac{7 p_{r}^{3}}{96 r}-\frac{7}{32} r^{2} p_{r} \dot{p}_{r}^{2}\right)\right]+\left[\frac{3 p_{\phi}}{32} r^{5} \dot{p}_{r}^{4}-\frac{p_{\phi}}{8} r^{2} p_{r}^{2} \dot{p}_{r}^{2}+\frac{p_{\phi} p_{r}^{4}}{48 r}\right.\right. \\
& \left.+i\left(\frac{r^{4}}{12} p_{r} \dot{p}_{r}^{3}-\frac{r}{96} p_{r}^{3} \dot{p}_{r}\right)\right]+\left[\frac{31}{384} p_{\phi} r p_{r}^{4} \dot{p}_{r}-\frac{173 p_{\phi} r^{7} \dot{p}_{r}^{5}}{1920}-\frac{1}{192} p_{\phi} r^{4} p_{r}^{2} \dot{p}_{r}^{3}+i\left(\frac{r^{6} p_{r}}{768} \dot{p}_{r}^{4}-\frac{49}{384} r^{3} p_{r}^{3} \dot{p}_{r}^{2}+\frac{89 p_{r}^{5}}{3840}\right)\right] \\
& \left.+\left[\frac{97 p_{\phi} r^{9} \dot{p}_{r}^{6}}{1152}+\frac{1}{16} p_{\phi} r^{6} p_{r}^{2} \dot{p}_{r}^{4}-\frac{47}{384} p_{\phi} r^{3} p_{r}^{4} \dot{p}_{r}^{2}+\frac{p_{\phi} p_{r}^{6}}{96}+i\left(-\frac{1}{64} r^{8} p_{r} \dot{p}_{r}^{5}+\frac{137 r^{5} p_{r}^{3} \dot{p}_{r}^{3}}{1152}-\frac{23}{640} r^{2} p_{r}^{5} \dot{p}_{r}\right)\right]\right\}, \\
& \hat{H}_{\text {tail }}^{20}=\frac{\pi}{\sqrt{6} c^{3}}\left\{\frac{p_{\phi} \dot{p}_{r}}{r}-p_{\phi} r \dot{p}_{r}^{2}+\left[\frac{3}{4} p_{\phi} r^{3} \dot{p}_{r}^{3}-\frac{1}{4} p_{\phi} p_{r}^{2} \dot{p}_{r}\right]+\left[-\frac{7}{12} p_{\phi} r^{5} \dot{p}_{r}^{4}-\frac{p_{\phi} p_{r}^{4}}{6 r}\right]+\left[\frac{95}{192} p_{\phi} r^{7} \dot{p}_{r}^{5}+\frac{13}{96} p_{\phi} r^{4} p_{r}^{2} \dot{p}_{r}^{3}\right.\right. \\
& \left.\left.+\frac{11}{192} p_{\phi} r p_{r}^{4} \dot{p}_{r}\right]+\left[-\frac{139}{320} p_{\phi} r^{9} \dot{p}_{r}^{6}-\frac{11}{96} p_{\phi} r^{6} p_{r}^{2} \dot{p}_{r}^{4}+\frac{3}{64} p_{\phi} r^{3} p_{r}^{4} \dot{p}_{r}^{2}-\frac{p_{\phi} p_{r}^{6}}{480}\right]\right\}, \\
& \hat{H}_{\text {tail }}^{33}=-\frac{9 i \pi \delta}{4 c^{4}} \sqrt{\frac{15}{14}}\left\{\frac{1}{r^{3}}+\left[\frac{23 \dot{p}_{r}}{27 r}+\frac{10 i p_{\phi} p_{r}}{27 r^{3}}\right]-\frac{2 p_{r}^{2}}{27 r^{2}}+\left[i\left(\frac{25}{432} p_{\phi} p_{r}^{3} \dot{p}_{r}-\frac{25}{432} p_{\phi} r^{3} p_{r} \dot{p}_{r}^{3}\right)-\frac{25 r^{5} \dot{p}_{r}^{4}}{1728}+\frac{25}{288} r^{2} p_{r}^{2} \dot{p}_{r}^{2}\right.\right. \\
& \left.-\frac{25 p_{r}^{4}}{1728 r}\right]+\left[i\left(\frac{109 p_{\phi} r^{5} p_{r} \dot{p}_{r}^{4}}{2592}+\frac{41 p_{\phi} r^{2} p_{r}^{3} \dot{p}_{r}^{2}}{1296}-\frac{41 p_{\phi} p_{r}^{5}}{12960 r}\right)+\frac{293 r^{7} \dot{p}_{r}^{5}}{25920}+\frac{41 r^{4} p_{r}^{2} \dot{\dot{p}}_{r}^{3}}{1296}-\frac{157 r p_{r}^{4} \dot{p}_{r}}{5184}\right]+\left[\frac{2845 r^{3} p_{r}^{4} \dot{p}_{r}^{2}}{41472}\right. \\
& \left.\left.-\frac{1561 r^{9} \dot{p}_{r}^{6}}{207360}-\frac{775 r^{6} p_{r}^{2} \dot{p}_{r}^{4}}{13824}-\frac{653 p_{r}^{6}}{69120}+i\left(\frac{4211 p_{\phi} r p_{r}^{5} \dot{p}_{r}}{103680}-\frac{2173 p_{\phi} r^{7} p_{r} \dot{p}_{r}^{5}}{103680}-\frac{307 p_{\phi} r^{4} p_{r}^{3} \dot{p}_{r}^{3}}{3456}\right)\right]\right\}, \\
& \hat{H}_{\text {tail }}^{31}=\frac{i \pi \delta}{12 \sqrt{14} c^{4}}\left\{\frac{1}{r^{3}}+\left[\frac{10 i p_{\phi} p_{r}}{r^{3}}-\frac{11 \dot{p}_{r}}{r}\right]-\left[\frac{11 i p_{\phi} \dot{p}_{r} p_{r}}{2 r}+\frac{13 p_{r}^{2}}{4 r^{2}}+\frac{11 r \dot{p}_{r}^{2}}{4}\right]+\left[i\left(6 p_{\phi} r p_{r} \dot{p}_{r}^{2}-\frac{p_{\phi} p_{r}^{3}}{6 r^{2}}\right)+\frac{35}{12} r^{3} \dot{p}_{r}^{3}\right.\right. \\
& \left.-\frac{p_{r}^{2} \dot{p}_{r}}{2}\right]+\left[i\left(\frac{45}{16} p_{\phi} p_{r}^{3} \dot{p}_{r}-\frac{61}{16} p_{\phi} r^{3} p_{r} \dot{p}_{r}^{3}\right)-\frac{269}{192} r^{5} \dot{p}_{r}^{4}+\frac{37}{32} r^{2} p_{r}^{2} \dot{p}_{r}^{2}-\frac{63 p_{r}^{4}}{64 r}\right]+\left[\frac{643}{960} r^{7} \dot{p}_{r}^{5}+\frac{5}{16} r^{4} p_{r}^{2} \dot{p}_{r}^{3}\right. \\
& \left.+\frac{101}{192} r p_{r}^{4} \dot{p}_{r}+i\left(\frac{83}{32} p_{\phi} r^{5} p_{r} \dot{p}_{r}^{4}-\frac{43}{48} p_{\phi} r^{2} p_{r}^{3} \dot{p}_{r}^{2}+\frac{379 p_{\phi} p_{r}^{5}}{480 r}\right)\right]+\left[\frac{317}{768} r^{3} p_{r}^{4} \dot{p}_{r}^{2}-\frac{5039 r^{9} \dot{p}_{r}^{6}}{11520}-\frac{289}{768} r^{6} p_{r}^{2} \dot{p}_{r}^{4}-\frac{297 p_{r}^{6}}{1280}\right. \\
& \left.\left.+i\left(\frac{151}{576} p_{\phi} r^{4} p_{r}^{3} \dot{p}_{r}^{3}-\frac{4267 p_{\phi} r^{7} p_{r} \dot{p}_{r}^{5}}{1920}+\frac{271}{640} p_{\phi} r p_{r}^{5} \dot{p}_{r}\right)\right]\right\} \text {. }
\end{aligned}
$$

\section{Modes with odd $\ell+m$}

The Newtonian order current quadrupole moment is given by [96]

$$
J^{i j}=-\delta \mu r^{2} n_{k} v_{l} \epsilon^{k l\langle i} n^{j\rangle}=-\delta r p_{\phi} \hat{e}_{z}^{\langle i} n^{j\rangle},
$$

where $\hat{e}_{z}^{i}$ is the unit vector in the $z$ direction. The term $e_{z}^{\langle i} n^{j\rangle}$ can be expressed in terms of $\mathcal{Y}_{21}^{i j}$, as was done in Ref. [124], by defining the complex vector

$$
\zeta^{i}=e_{x}^{i}+i e_{y}^{i}
$$

which leads to

$$
\mathcal{Y}_{21}^{i j}=-\frac{1}{2} \sqrt{\frac{15}{2 \pi}} \zeta^{\langle i} e_{z}^{j\rangle}
$$

Since, for equatorial orbits, $n^{i}=\cos \phi \hat{e}_{x}^{i}+\sin \phi \hat{e}_{y}^{i}$ and $\lambda^{i}=-\sin \phi \hat{e}_{x}^{i}+\cos \phi \hat{e}_{y}^{i}$, we obtain

$$
n^{i}+i \lambda^{i}=e^{-i \phi} \zeta^{i}
$$

Hence,

$$
\begin{aligned}
e_{z}^{\langle i} n^{j\rangle} & =\operatorname{Re}\left[\mathrm{e}^{-i \phi} e_{z}^{\langle i} \zeta^{j\rangle}\right] \\
& =-2 \sqrt{\frac{2 \pi}{15}} \operatorname{Re}\left[\mathrm{e}^{-i \phi} \mathcal{Y}_{21}^{i j}\right] \\
& =-\sqrt{\frac{2 \pi}{15}}\left(\mathrm{e}^{-i \phi} \mathcal{Y}_{21}^{i j}+\mathrm{e}^{i \phi} \overline{\mathcal{Y}}_{21}^{i j}\right) .
\end{aligned}
$$

Since $V_{i j}$ is contracted with $\overline{\mathcal{Y}}_{i j}^{\ell m}$ in Eq. (74), and $\overline{\mathcal{Y}}_{i j}^{\ell m} \overline{\mathcal{Y}}_{\ell m}^{i j}=0$, only the term with $\mathcal{Y}_{21}^{i j}$ in the above equation contributes to the modes. Thus, we only need to consider the following part of the current quadrupole

$$
J^{i j}=\mu \delta \sqrt{\frac{2 \pi}{15}} \mathcal{Y}_{21}^{i j} p_{\phi} r e^{-i \phi}+\cdots .
$$

Then, we follow the same steps as in the previous subsection. Decomposing the phase into $\phi=\omega_{\phi} t+\Delta \phi$ leads to 


$$
J^{i j}=\mu \delta \sqrt{\frac{2 \pi}{15}} \mathcal{Y}_{21}^{i j} p_{\phi} \mathrm{e}^{-i \psi_{\phi} J_{11}}+\cdots
$$

with

$$
J_{11}=r \mathrm{e}^{-i \Delta \phi}=\sum_{k=-\infty}^{\infty} J_{11 k} \mathrm{e}^{-i k \psi_{r}}
$$

and

$$
\begin{aligned}
J_{11 k} & =\frac{1}{2 \pi} \int_{0}^{2 \pi} d \psi_{r} J_{11} \mathrm{e}^{i k \psi_{r}} \\
& =\frac{\omega_{r}}{2 \pi u_{p}^{5 / 2}} \int_{0}^{2 \pi} \frac{d \chi}{(1+e \cos \chi)^{3}} \mathrm{e}^{-i \Delta \phi} \mathrm{e}^{i k \psi_{r}} .
\end{aligned}
$$

Thus, the current quadrupole source moment can be expressed as

$$
J^{i j}=\mu \delta \sqrt{\frac{2 \pi}{15}} \mathcal{Y}_{21}^{i j} p_{\phi} \sum_{k=-\infty}^{\infty} J_{11 k} \mathrm{e}^{-i\left(k \psi_{r}+\psi_{\phi}\right)}+\cdots,
$$

and the current quadrupole radiative moment

$$
\begin{aligned}
V_{i j}^{\mathrm{tail}}= & \frac{2 M \mu \delta}{c^{3}} \sqrt{\frac{2 \pi}{15}} \mathcal{Y}_{21}^{i j} p_{\phi} \sum_{k=-\infty}^{\infty} J_{11 k} \Omega_{1 k}^{4} \\
& \times \mathrm{e}^{-i\left(k \psi_{r}+\psi_{\phi}\right)} \mathcal{I}\left(\Omega_{1 k}\right),
\end{aligned}
$$

leading to the $(2,1)$ mode

$$
\begin{aligned}
h_{\text {tail }}^{21}= & \frac{16 i}{3} \sqrt{\frac{\pi}{5}} \frac{\delta M}{R c^{8}} \mathrm{e}^{-i \phi} p_{\phi} \sum_{k=-\infty}^{\infty} J_{11 k} \Omega_{1 k}^{4} \mathrm{e}^{i \chi} \\
& \times \mathrm{e}^{-i(k+1) \psi_{r}} \mathcal{I}\left(\Omega_{1 k}\right),
\end{aligned}
$$

with $p_{\phi}=1 / \sqrt{u_{p}}=\sqrt{\left(1-e^{2}\right) / x}$. Expanding in eccentricity yields

$$
\begin{aligned}
\hat{H}_{\text {tail }}^{21}= & \frac{i \pi \delta}{3 c^{4}} x^{3}\left[1+e\left(3 \mathrm{e}^{-i \chi}+\mathrm{e}^{i \chi}\right)+e^{2}\left(3 \mathrm{e}^{-2 i \chi}+\frac{1}{4} \mathrm{e}^{2 i \chi}+6\right)+e^{3}\left(\frac{45 \mathrm{e}^{-i \chi}}{4}+4 \mathrm{e}^{i \chi}+\frac{5}{4} \mathrm{e}^{-3 i \chi}+\frac{1}{6} \mathrm{e}^{3 i \chi}\right)\right. \\
& +e^{4}\left(\frac{19}{2} \mathrm{e}^{-2 i \chi}+\frac{25}{24} \mathrm{e}^{2 i \chi}+\frac{3}{16} \mathrm{e}^{-4 i x}+\frac{17}{192} \mathrm{e}^{4 i \chi}+\frac{493}{32}\right)+e^{5}\left(\frac{2375 \mathrm{e}^{-i \chi}}{96}+\frac{865 \mathrm{e}^{i \chi}}{96}+\frac{15}{4} \mathrm{e}^{-3 i \chi}+\frac{91}{192} \mathrm{e}^{3 i \chi}-\frac{7}{960} \mathrm{e}^{5 i \chi}\right) \\
& \left.+e^{6}\left(\frac{29957 \mathrm{e}^{-2 i \chi}}{1536}+\frac{593}{256} \mathrm{e}^{2 i \chi}+\frac{9}{16} \mathrm{e}^{-4 i \chi}+\frac{241}{960} \mathrm{e}^{4 i \chi}+\frac{37 \mathrm{e}^{6 i \chi}}{23040}+\frac{8417}{288}\right)\right]
\end{aligned}
$$

which is in agreement with the results of Ref. [122]. In terms of $\left(r, p_{r}, p_{\phi}, \dot{p}_{r}\right)$, we obtain

$$
\begin{aligned}
\hat{H}_{\text {tail }}^{21}= & \frac{i \pi \delta}{3 c^{4}}\left\{\frac{1}{r^{3}}+\left[\frac{\dot{p}_{r}}{r}-\frac{2 i p_{\phi} p_{r}}{r^{3}}\right]+\left[\frac{i p_{\phi} \dot{p}_{r} p_{r}}{2 r}-\frac{p_{r}^{2}}{4 r^{2}}+\frac{1}{4} r \dot{p}_{r}^{2}\right]+\left[-\frac{i p_{\phi} p_{r}^{3}}{6 r^{2}}-\frac{1}{12} r^{3} \dot{p}_{r}^{3}-\frac{1}{2} \dot{p}_{r} p_{r}^{2}\right]\right. \\
& +\left[i\left(\frac{5}{16} p_{\phi} p_{r}^{3} \dot{p}_{r}-\frac{5}{16} p_{\phi} r^{3} p_{r} \dot{p}_{r}^{3}\right)-\frac{1}{192} 5 r^{5} \dot{p}_{r}^{4}+\frac{13}{32} r^{2} p_{r}^{2} \dot{p}_{r}^{2}-\frac{7 p_{r}^{4}}{64 r}\right] \\
& +\left[i\left(\frac{11}{32} p_{\phi} r^{5} p_{r} \dot{p}_{r}^{4}-\frac{19}{48} p_{\phi} r^{2} p_{r}^{3} \dot{p}_{r}^{2}+\frac{19 p_{\phi} p_{r}^{5}}{480 r}\right)+\frac{43}{960} r^{7} \dot{p}_{r}^{5}-\frac{3}{16} r^{4} p_{r}^{2} \dot{p}_{r}^{3}+\frac{29}{192} r p_{r}^{4} \dot{p}_{r}\right] \\
& \left.+\left[i\left(-\frac{511 p_{\phi} r^{7} p_{r} \dot{p}_{r}^{5}}{1920}+\frac{115}{576} p_{\phi} r^{4} p_{r}^{3} \dot{p}_{r}^{3}-\frac{61}{640} p_{\phi} r p_{r}^{5} \dot{p}_{r}\right)-\frac{79 r^{9} \dot{p}_{r}^{6}}{2304}-\frac{13}{768} r^{6} p_{r}^{2} \dot{p}_{r}^{4}+\frac{17}{768} r^{3} p_{r}^{4} \dot{p}_{r}^{2}+\frac{59 p_{r}^{6}}{1280}\right]\right\} .
\end{aligned}
$$

\section{Aligned-spin contributions}

The spin contributions to the modes were derived for circular orbits in Refs. $[116,120,125]$. To derive the spin part of the modes to $2 \mathrm{PN}$ for eccentric orbits, we use the source moments from Refs. [116,121], which are in harmonic coordinates and in terms of the covariant SSC.
Differentiating the source moments to obtain the radiative moments (82), and plugging them into Eq. (73), we obtain the modes listed in Appendix C. Transforming from harmonic to EOB coordinates, and from the covariant to the NW SSC using the transformations in Appendix A, we obtain the following spin contributions to the modes: 


$$
\begin{aligned}
& \hat{H}_{\mathrm{spin}}^{22}=\frac{1}{c^{3}}\left[\frac{\chi_{1}}{12 r^{3}}\left[(6 \delta+\nu+6) p_{\phi}+2 i(3 \delta-\nu+3) r p_{r}\right]+\frac{\chi_{2}}{12 r^{3}}\left[(-6 \delta+\nu+6) p_{\phi}-2 i(3 \delta+\nu-3) r p_{r}\right]\right] \\
& +\frac{1}{4 c^{4} r^{3}}\left\{\chi_{1}^{2}\left[3 C_{1 \mathrm{ES}^{2}} X_{1}^{2}-X_{1}^{4}\left(2 p^{2} r+1\right)\right]+\chi_{2}^{2}\left[3 C_{2 \mathrm{ES}^{2}} X_{2}^{2}-X_{2}^{4}\left(2 p^{2} r+1\right)\right]-2 \nu \chi_{1} \chi_{2}\left(\nu-3+2 \nu p^{2} r\right)\right\}, \\
& \hat{H}_{\mathrm{spin}}^{21}=\frac{i}{4 c^{2} r^{2}}\left[-(1+\delta) \chi_{1}+(1-\delta) \chi_{2}\right]+\frac{\chi_{1}}{84 c^{4} r^{2}}\left[\frac{i p_{\phi}^{2}}{r^{2}}(43 \nu \delta-42 \delta+153 \nu-42)+\frac{p_{r} p_{\phi}}{r}(3 \delta(2 \nu+49)-104 \nu+147)\right. \\
& \left.+\frac{i}{2} p_{r}^{2}(\delta(38 \nu+105)+74 \nu+105)+\frac{i}{r}(63 \delta-38 \nu \delta-74 \nu+63)\right]+\frac{\chi_{2}}{84 c^{4} r^{2}}\left[\frac{i p_{\phi}^{2}}{r^{2}}(43 \nu \delta-42 \delta-153 \nu+42)\right. \\
& \left.+\frac{p_{r} p_{\phi}}{r}(3 \delta(2 \nu+49)+104 \nu-147)+\frac{i}{2} p_{r}^{2}(38 \nu \delta+105 \delta-74 \nu-105)+\frac{i}{r}(63 \delta-38 \nu \delta+74 \nu-63)\right] \text {, }
\end{aligned}
$$

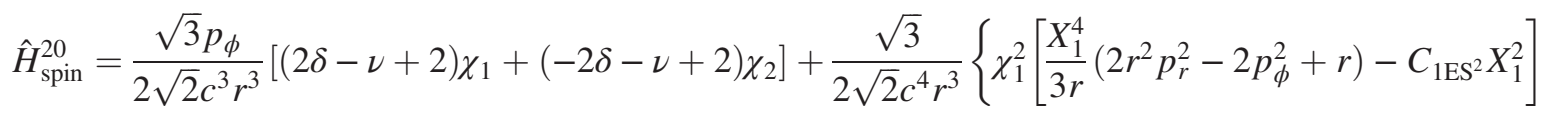

$$
\begin{aligned}
& \left.+\chi_{2}^{2}\left[\frac{X_{2}^{4}}{3 r}\left(2 r^{2} p_{r}^{2}-2 p_{\phi}^{2}+r\right)-C_{2 \mathrm{ES}^{2}} X_{2}^{2}\right]+\frac{2 \nu \chi_{1} \chi_{2}}{3}\left[\nu-3-2 \nu \frac{p_{\phi}^{2}}{r}+2 \nu r p_{r}^{2}\right]\right\} \\
& \hat{H}_{\text {spin }}^{30}=\frac{-i \nu p_{r}}{\sqrt{42} c^{3} r^{2}}\left(\chi_{1}+\chi_{2}\right) \\
& \hat{H}_{\mathrm{spin}}^{31}=\frac{1}{24 \sqrt{14} c^{4} r^{2}}\left\{\chi _ { 1 } \left[\frac{i p_{\phi}^{2}}{2 r^{2}}(55 \nu \delta-96 \delta+375 \nu-96)+i\left(p_{r}^{2}-\frac{2}{r}\right)(2 \nu \delta-6 \delta+23 \nu-6)\right.\right. \\
& \left.+\frac{p_{r} p_{\phi}}{r}(-6 \nu \delta+30 \delta-127 \nu+30)\right]+\chi_{2}\left[\frac{i p_{\phi}^{2}}{2 r^{2}}(55 \nu \delta-96 \delta-375 \nu+96)+\frac{p_{r} p_{\phi}}{r}(-6 \nu \delta+30 \delta+127 \nu-30)\right. \\
& \left.\left.+i\left(p_{r}^{2}-\frac{2}{r}\right)(2 \nu \delta-6 \delta-23 \nu+6)\right]\right\} \\
& \hat{H}_{\text {spin }}^{32}=\frac{\nu}{6 c^{3} r^{3}} \sqrt{\frac{5}{7}}\left(4 p_{\phi}+i r p_{r}\right)\left(\chi_{1}+\chi_{2}\right) \\
& \hat{H}_{\text {spin }}^{33}=\frac{\sqrt{5}}{8 \sqrt{42} c^{4} r^{2}}\left\{\left[-\frac{23 i(\delta+1) \nu p_{\phi}^{2}}{2 r^{2}}+\frac{(2 \nu \delta+6 \delta-19 \nu+6) p_{r} p_{\phi}}{r}+i\left(\frac{2}{r}-p_{r}^{2}\right)(2 \nu \delta-6 \delta+23 \nu-6)\right] \chi_{1}\right. \\
& \left.+\left[-\frac{23 i(\delta-1) \nu p_{\phi}^{2}}{2 r^{2}}+\frac{(2 \delta \nu+6 \delta+19 \nu-6) p_{r} p_{\phi}}{r}+i\left(\frac{2}{r}-p_{r}^{2}\right)(2 \delta \nu-6 \delta-23 \nu+6)\right] \chi_{2}\right\} \\
& \hat{H}_{\mathrm{spin}}^{41}=-i \sqrt{\frac{5}{2}} \frac{\nu}{336 c^{4} r^{4}}\left(-10 i r p_{r} p_{\phi}+6 r^{2} p_{r}^{2}-12 r+11 p_{\phi}^{2}\right)\left[(\delta-1) \chi_{1}+(\delta+1) \chi_{2}\right], \\
& \hat{H}_{\mathrm{spin}}^{43}=\sqrt{\frac{5}{14}} \frac{\nu}{48 c^{4} r^{4}}\left(10 r p_{r} p_{\phi}+2 i r^{2} p_{r}^{2}-4 i r-23 i p_{\phi}^{2}\right)\left[(\delta-1) \chi_{1}+(\delta+1) \chi_{2}\right] .
\end{aligned}
$$

The circular-orbit limit of these modes, when expressed in terms of the orbital frequency, agrees with the results of Refs. [116,125]. The spin contributions to the $(2,2),(2,1)$, and $(3,3)$ modes for eccentric orbits were calculated in Ref. [82]; however, we find a small disagreement with their results for the SO part.

\footnotetext{
${ }^{4}$ The difference between the modes in Ref. [82] (denoted with a bar) and the modes in Eq. (117) (with $C_{1 \mathrm{ES}^{2}}=C_{2 \mathrm{ES}^{2}}=1$ ) is given by $\hat{\bar{H}}_{\text {spin }}^{22}-\hat{H}_{\text {spin }}^{22}=\frac{i \nu p_{r}}{2 c^{3} r^{2}}\left(\chi_{1}+\chi_{2}\right), \quad \hat{\bar{H}}_{\text {spin }}^{21}-\hat{H}_{\text {spin }}^{21}=\frac{i \delta \nu p_{r}}{6 c^{4} r^{3}}\left(r p_{r}+i p_{\phi}\right)\left(\chi_{1}+\chi_{2}\right), \quad \hat{\bar{H}}_{\text {spin }}^{33}-\hat{H}_{\text {spin }}^{33}=\frac{\sqrt{5} \delta \nu p_{r}}{8 \sqrt{42} c^{4} r^{3}}\left(17 p_{\phi}+5 i r p_{r}\right)\left(\chi_{1}+\chi_{2}\right)$, which is likely due to the coordinate/SSC transformations detailed in Appendix A.
} 


\section{Factorized modes}

The quasicircular waveform modes used in SEOBNRV4HM are factorized as follows $[70,83,126,127]$ :

$$
h_{\ell m}^{F, \mathrm{qc}}=h_{\ell m}^{N, \mathrm{qc}} \hat{S}_{\mathrm{eff}}^{\mathrm{qc}} T_{\ell m}^{\mathrm{qc}} e^{i \delta_{\ell m}} f_{\ell m}^{\mathrm{qc}},
$$

where $h_{\ell m}^{N, q c}$ is the Newtonian part of the mode, $\hat{S}_{\text {eff }}^{\mathrm{qc}}$ is an effective source term given by

$$
\hat{S}_{\text {eff }}^{\mathrm{qc}}=\left\{\begin{array}{ll}
\hat{H}_{\mathrm{eff}}\left(v_{\Omega}\right) & \ell+m \text { even } \\
\hat{L}_{\mathrm{eff}} \equiv v_{\Omega} p_{\phi}\left(v_{\Omega}\right) & \ell+m \text { odd }
\end{array},\right.
$$

$T_{\ell m}^{\mathrm{qc}}$ resums the infinite number of "leading logarithms" entering the tail effects, $\delta_{\ell m}$ contains the part of the tail not included in $T_{\ell m}^{\mathrm{qc}}$, and $f_{\ell m}$ contains PN corrections such that the expansion of $h_{\ell m}^{F, \mathrm{qc}}$ agrees with the known PN expansion of the modes. See Refs. [70,83] for more details and for expressions of these terms.

We include the eccentric corrections in the factorized modes as follows:

$$
\begin{aligned}
h_{\ell 0}^{\mathrm{F}} & =\hat{S}_{\mathrm{eff}}\left(1+T_{\ell 0}^{\mathrm{ecc}}\right) f_{\ell 0}^{\mathrm{ecc}}, \\
h_{\ell m}^{\mathrm{F}} & =h_{\ell m}^{N, \mathrm{qc}} \hat{S}_{\mathrm{eff}}\left(T_{\ell m}^{\mathrm{qc}}+T_{\ell m}^{\mathrm{ecc}}\right) e^{i \delta_{\ell m}}\left(f_{\ell m}^{\mathrm{qc}}+f_{\ell m}^{\mathrm{ecc}}\right),
\end{aligned}
$$

where the effective source term is given by

$$
\hat{S}_{\mathrm{eff}}=\left\{\begin{array}{ll}
\hat{H}_{\mathrm{eff}}\left(r, p_{r}, p_{\phi}\right) & \ell+m \text { even } \\
\hat{L}_{\mathrm{eff}} \equiv v_{\Omega} p_{\phi} & \ell+m \text { odd }
\end{array},\right.
$$

$T_{\ell m}^{\mathrm{ecc}}$ contains the eccentric corrections to the hereditary contributions, $\delta_{\ell m}$ is the same as in the quasicircular case, and $f_{\ell m}^{\text {ecc }}$ contains the eccentric corrections to the instantaneous contributions (both spinning and nonspinning, including the Newtonian part). For example, for the leading order of the $(2,2)$ mode, we obtain

$$
\begin{aligned}
f_{22}^{\mathrm{ecc}}= & \frac{1}{2\left(r^{2} \dot{p}_{r}+1\right)^{1 / 3}}\left[2+r^{2} \dot{p}_{r}-r p_{r}^{2}-2\left(r^{2} \dot{p}_{r}+1\right)^{1 / 3}\right. \\
& \left.+2 i p_{r} \sqrt{r^{3} \dot{p}_{r}+r}\right]+\cdots .
\end{aligned}
$$

For the tail part, we simplified the results of Sec. IV B and eliminated the gauge parameter by using a phase shift, which led to the circular part of the tail contribution to the $(2,2)$ mode simply being $2 \pi v_{\Omega}^{5}$; however, this phase redefinition is not done in SEOBNRV4HM, and the corresponding expression reads $v_{\Omega}^{5}\left(2 \pi+12 i \log \left(2 \epsilon v_{\Omega}\right)-\right.$ $\left.17 i / 3+12 i \gamma_{E} / 3\right)$. Therefore, when including the eccentric corrections in $T_{\ell m}^{\mathrm{ecc}}$, we assume that the phase redefinition was done only for the eccentric part and keep using the same circular part as in SEOBNRV4HM. In addition, since we expanded the tail part in eccentricity to $\mathcal{O}\left(e^{6}\right)$, when factorizing the modes as in Eq. (120) and writing the quasicircular part in terms of frequency, we reexpand $T_{l m}^{\mathrm{ecc}}$ in eccentricity (or $p_{r}$ and $\dot{p}_{r}$ ). For example, for the $(2,2)$ mode, we obtain

$$
T_{22}^{\mathrm{ecc}}=-\frac{\pi}{4 r}\left[4 r^{3 / 2} \dot{p}_{r}+i p_{r}\left(r^{2} \dot{p}_{r}+6\right)+2 \sqrt{r} p_{r}^{2}+\mathcal{O}\left(p_{r}^{3}\right)\right] .
$$

The full expressions for $T_{l m}^{\mathrm{ecc}}$ and $f_{l m}^{\mathrm{ecc}}$ are provided in the Supplemental Material [88].

\section{CONCLUSIONS}

Extending the waveform models used today in GW astronomy from quasicircular to eccentric orbits is important for future observations with LIGO, Virgo, and KAGRA detectors [21], and with new facilities on the ground (Cosmic Explorer and Einstein Telescope), and in space (LISA). In fact, sources with non-negligible eccentricity might come into reach of observations soon and should routinely be included in searches and parameter inference. While this presents a challenge for waveform modeling and data analysis, it also offers the unique opportunity to unveil the formation channels of compact binaries and probe their environment (through eccentricity measurements). In this paper, we constructed an EOB waveform model for eccentric binaries. For this purpose, we obtained analytical results for the RR force and waveform modes to $2 \mathrm{PN}$ order, including the leading-order tail effects, and SO and SS couplings for aligned spins.

In particular, we first derived the RR force for eccentric orbits in PN expanded form, and then we recast it in a form that it can be directly incorporated in the quasicircular RR force employed in the SEOBNRV4HM [74,83] model, currently used in LIGO/Virgo analyses [1]. We then obtained initial conditions for the binary evolution which generalize those from Ref. [94] to eccentric orbits, and which allow starting the binary's evolution from a specified initial frequency at periastron and an initial eccentricity (in the Keplerian parametrization). We also calculated all the waveform modes that contribute up to $2 \mathrm{PN}$ order relative to the leading order of the $(2,2)$ mode. It should be noted that the $(\ell, 0)$ modes are proportional to the eccentricity and are hence important for eccentric orbits, especially the $(2,0)$ mode since it starts at the same PN order as the $(2,2)$ mode. Also the gravitational modes were rewritten in a factorized form to be straightforwardly implemented in the SEOBNRV4HM model.

Our results for the RR force and modes are valid for moderate to high eccentricities during the inspiral phase, since we do not use an eccentricity expansion except for the tail part, which is known analytically as an infinite series expansion. We provided expressions for the tail part in an expansion to $\mathcal{O}\left(e^{6}\right)$, but we checked that expanding to $\mathcal{O}\left(e^{10}\right)$ produces negligible difference on the waveform even for high eccentricities $(\lesssim 0.9)$. If results for $e$ close to 
1 are needed, one could calculate the series expansion for the tail part numerically, or use analytical resummation methods as was done in Refs. [31,98].

We are currently incorporating the eccentric RR force and gravitational modes of this paper in the inspiralmerger-ringdown quasicircular-orbit SEOBNRV4HM waveform model (SEOBNRV4EHM [128]) and validating it against NR simulations with eccentricity. We leave to future work the extension of the model to higher PN orders and the inclusion of spin precession.

\section{ACKNOWLEDGMENTS}

We are grateful to Serguei Ossokine, Harald Pfeiffer, Antoni Ramos-Buades, Hannes Rüter, and Maarten van de Meent for helpful discussions. We also thank Marco Stella, Marta Orselli, and Andrea Placidi for pointing out typos in Eq. (A4).

\section{APPENDIX A: COORDINATE TRANSFORMATION FROM HARMONIC TO EOB COORDINATES}

The coordinate transformation from harmonic to $\mathrm{EOB}$ coordinates with no spin is given in Appendix A of
Ref. [78]. In this Appendix, we include LO SO and SS contributions to the transformation. We label harmonic, $\mathrm{ADM}$, and EOB coordinates by $\left(\boldsymbol{x}_{h}, \boldsymbol{v}_{h}\right),\left(\boldsymbol{x}_{a}, \boldsymbol{p}_{a}\right)$, and $(\boldsymbol{x}, \boldsymbol{p})$, respectively.

\section{ADM to EOB transformation}

To find the canonical transformation from the ADM Hamiltonian with LO SO and SS using the NW SSC (see, e.g., Refs. [129-131]) and the 2PN expansion of the EOB Hamiltonian of Ref. [50], we write an ansatz with unknown coefficients for the generating function $G(\boldsymbol{x}, \boldsymbol{p})$, perform the following transformation on the ADM Hamiltonian [45]:

$$
\begin{aligned}
& x_{a}^{i}=x^{i}+\frac{\partial G}{\partial p_{i}}-\frac{\partial G}{\partial x^{j}} \frac{\partial^{2} G}{\partial p_{j} \partial p_{i}}+\mathcal{O}\left(\frac{1}{c^{6}}\right) \\
& p_{a}^{i}=p^{i}-\frac{\partial G}{\partial x_{i}}+\frac{\partial G}{\partial x^{j}} \frac{\partial^{2} G}{\partial p_{j} \partial x^{i}}+\mathcal{O}\left(\frac{1}{c^{6}}\right),
\end{aligned}
$$

and match it to the EOB Hamiltonian to solve for the unknowns.

The result for the generating function is given by

$$
\begin{aligned}
G(x, p)= & \frac{p_{r}}{c^{2}}\left[-1-\frac{\nu}{2}+\frac{1}{2} \nu p^{2} r\right]+\frac{p_{r}}{c^{4}}\left[\frac{1}{8} \nu(3 \nu-1) p^{4} r-\frac{1}{8} \nu(\nu+14) p^{2}-\frac{\nu^{2}-7 \nu+1}{4 r}+\frac{1}{8} \nu^{2} p_{r}^{2}\right] \\
& +\frac{\nu^{2}}{2 c^{4} r}\left[p_{r}\left(\hat{\boldsymbol{S}}_{1}+\hat{\boldsymbol{S}}_{2}\right)^{2}-\left(\boldsymbol{n} \cdot \hat{\boldsymbol{S}}_{1}+\boldsymbol{n} \cdot \hat{\boldsymbol{S}}_{2}\right)\left(\boldsymbol{p} \cdot \hat{\boldsymbol{S}}_{1}+\boldsymbol{p} \cdot \hat{\boldsymbol{S}}_{2}\right)\right],
\end{aligned}
$$

which has no LO SO terms since the ADM and EOB Hamiltonian are the same at that order. This generating function yields

$$
\begin{aligned}
\boldsymbol{x}_{a}= & \boldsymbol{x}+\frac{1}{c^{2}}\left[\boldsymbol{x}\left(\frac{\nu p^{2}}{2}-\frac{\nu+2}{2 r}\right)+\nu r p_{r} \boldsymbol{p}\right]+\frac{1}{c^{4}}\left\{\boldsymbol{x}\left[\frac{3(\nu-2) \nu p^{2}}{8 r}-\frac{1}{8} \nu(\nu+1) p^{4}-\frac{\nu(5 \nu+16) p_{r}^{2}}{8 r}-\frac{\nu^{2}-7 \nu+1}{4 r^{2}}\right]\right. \\
& \left.+\boldsymbol{p} p_{r}\left[\frac{1}{2}(\nu-1) \nu p^{2} r+\frac{(\nu-10) \nu}{4}\right]+\frac{\nu^{2}}{2 r}\left[\left(\hat{\boldsymbol{S}}_{1}+\hat{\boldsymbol{S}}_{2}\right)^{2} \frac{\boldsymbol{x}}{r}-\left(\hat{\boldsymbol{S}}_{1}+\hat{\boldsymbol{S}}_{2}\right)\left(\boldsymbol{n} \cdot \hat{\boldsymbol{S}}_{1}+\boldsymbol{n} \cdot \hat{\boldsymbol{S}}_{2}\right)\right]\right\}, \\
\boldsymbol{p}_{a}= & \boldsymbol{p}+\frac{1}{c^{2}}\left[\boldsymbol{p}\left(\frac{\nu+2}{2 r}-\frac{\nu p^{2}}{2}\right)-\boldsymbol{x} \frac{(\nu+2) p_{r}}{2 r^{2}}\right]+\frac{1}{c^{4}}\left\{\boldsymbol{p}\left[\frac{1}{8} \nu(3 \nu+1) p^{4}-\frac{\nu(7 \nu+2) p^{2}}{8 r}+\frac{\nu(\nu+8) p_{r}^{2}}{8 r}+\frac{2 \nu^{2}-3 \nu+5}{4 r^{2}}\right]\right. \\
& +\boldsymbol{x} p_{r}\left[\frac{3(\nu-2) \nu p^{2}}{8 r^{2}}-\frac{3 \nu^{2}-10 \nu+6}{4 r^{3}}+\frac{3 \nu^{2} p_{r}^{2}}{8 r^{2}}\right]+\frac{\nu^{2} \boldsymbol{x}}{r^{3}}\left[\left(\hat{\boldsymbol{S}}_{1}+\hat{\boldsymbol{S}}_{2}\right)^{2} p_{r}-\left(\boldsymbol{n} \cdot \hat{\boldsymbol{S}}_{1}+\boldsymbol{n} \cdot \hat{\boldsymbol{S}}_{2}\right)\left(\boldsymbol{p} \cdot \hat{\boldsymbol{S}}_{1}+\boldsymbol{p} \cdot \hat{\boldsymbol{S}}_{2}\right)\right] \\
& \left.+\frac{\nu^{2}}{2 r^{2}}\left[-\left(\hat{\boldsymbol{S}}_{1}+\hat{\boldsymbol{S}}_{2}\right)^{2} \boldsymbol{p}+\left(\hat{\boldsymbol{S}}_{1}+\hat{\boldsymbol{S}}_{2}\right)\left(\boldsymbol{p} \cdot \hat{\boldsymbol{S}}_{1}+\boldsymbol{p} \cdot \hat{\boldsymbol{S}}_{2}\right)\right]\right\} .
\end{aligned}
$$

\section{Harmonic to EOB transformation}

The transformation from harmonic to ADM coordinates is given by Eq. (E1) of Ref. [78], which is independent of spin since the ADM and harmonic coordinates agree at LO SO and SS. Using that equation together with Eq. (A3), we obtain the following transformation from harmonic to EOB coordinates: 


$$
\begin{aligned}
\boldsymbol{x}_{h}= & \boldsymbol{x}+\frac{1}{c^{2}}\left[\boldsymbol{x}\left(\frac{\nu p^{2}}{2}-\frac{\nu+2}{2 r}\right)+\nu r p_{r} \boldsymbol{p}\right]+\frac{1}{c^{4}}\left\{\boldsymbol{x}\left[-\frac{1}{8} \nu(\nu+1) p^{4}+\frac{(3 \nu-1) \nu p^{2}}{8 r}-\frac{\nu(5 \nu+17) p_{r}^{2}}{8 r}-\frac{(\nu-19) \nu}{4 r^{2}}\right]\right. \\
& \left.+\boldsymbol{p} p_{r}\left[\frac{1}{4}(\nu-19) \nu+\frac{1}{2}(\nu-1) \nu p^{2} r\right]+\frac{\nu^{2}}{2 r}\left[\left(\hat{\boldsymbol{S}}_{1}+\hat{\boldsymbol{S}}_{2}\right)^{2} \frac{\boldsymbol{x}}{r}-\left(\hat{\boldsymbol{S}}_{1}+\hat{\boldsymbol{S}}_{2}\right)\left(\boldsymbol{n} \cdot \hat{\boldsymbol{S}}_{1}+\boldsymbol{n} \cdot \hat{\boldsymbol{S}}_{2}\right)\right]\right\} \\
\boldsymbol{v}_{h}= & \boldsymbol{p}+\frac{1}{c^{2}}\left[\boldsymbol{p}\left(\left(\nu-\frac{1}{2}\right) p^{2}-\frac{\nu+4}{2 r}\right)-\boldsymbol{x} \frac{(3 \nu+2) p_{r}}{2 r^{2}}\right]-\frac{1}{4 c^{3} r^{2}}\left[\boldsymbol{n} \times \hat{\boldsymbol{S}}_{1}(3-3 \delta+2 \nu)+\boldsymbol{n} \times \hat{\boldsymbol{S}}_{2}(3+3 \delta+2 \nu)\right] \\
& +\frac{1}{c^{4}}\left\{\boldsymbol{p}\left[\left(\frac{3}{8}-\nu\right) p^{4}+\frac{\left(7 \nu^{2}-41 \nu+8\right) p^{2}}{8 r}+\frac{\left(-15 \nu^{2}+29 \nu+8\right) p_{r}^{2}}{8 r}+\frac{-\nu^{2}+15 \nu+1}{2 r^{2}}\right]\right. \\
& +\boldsymbol{x} p_{r}\left[\frac{\left(4-7 \nu^{2}-23 \nu\right) p^{2}}{8 r^{2}}+\frac{4-3 \nu^{2}+9 \nu}{4 r^{3}}+\frac{3 \nu(5 \nu+1) p_{r}^{2}}{8 r^{2}}\right]+\frac{\nu^{2}}{2 r^{2}}\left[\left(\hat{\boldsymbol{S}}_{1}+\hat{\boldsymbol{S}}_{2}\right)\left(\boldsymbol{p} \cdot \hat{\boldsymbol{S}}_{1}+\boldsymbol{p} \cdot \hat{\boldsymbol{S}}_{2}\right)-\left(\hat{\boldsymbol{S}}_{1}+\hat{\boldsymbol{S}}_{2}\right)^{2} \boldsymbol{p}\right] \\
& \left.+\frac{\nu^{2} \boldsymbol{x}}{r^{3}}\left[\left(\hat{\boldsymbol{S}}_{1}+\hat{\boldsymbol{S}}_{2}\right)^{2} p_{r}-\left(\boldsymbol{n} \cdot \hat{\boldsymbol{S}}_{1}+\boldsymbol{n} \cdot \hat{\boldsymbol{S}}_{2}\right)\left(\boldsymbol{p} \cdot \hat{\boldsymbol{S}}_{1}+\boldsymbol{p} \cdot \hat{\boldsymbol{S}}_{2}\right)\right]\right\},
\end{aligned}
$$

and for the scalars $(\phi, r, \dot{\phi}, \dot{r})$, we obtain

$$
\begin{aligned}
\phi_{h}= & \phi+\frac{p_{\phi} \nu p_{r}}{c^{2} r}+\frac{p_{r} p_{\phi}}{c^{4}}\left[\frac{3(\nu-5) \nu}{4 r^{2}}-\frac{\nu p^{2}}{2 r}-\frac{\nu^{2} p_{r}^{2}}{r}\right], \\
r_{h}= & r+\frac{1}{c^{2}}\left(\frac{\nu}{2} p^{2} r+\nu r p_{r}^{2}-1-\frac{\nu}{2}\right)+\frac{1}{c^{4}}\left[\frac{\nu}{8}(3 \nu-1) p^{2}-\frac{\nu}{8}(\nu+1) p^{4} r-\frac{\nu}{4 r}(\nu-19)+\frac{\nu}{2}(2 \nu-1) p^{2} p_{r}^{2} r\right. \\
& \left.-\frac{\nu}{8}(3 \nu+55) p_{r}^{2}-\frac{1}{2} \nu^{2} r p_{r}^{4}+\frac{1}{2 r}\left(X_{1}^{4} \chi_{1}^{2}+2 \nu^{2} \chi_{1} \chi_{2}+X_{2}^{4} \chi_{2}^{2}\right)\right], \\
\dot{\phi}_{h}= & \frac{p_{\phi}}{r^{2}}+\frac{p_{\phi}}{c^{2} r^{2}}\left[\frac{\nu-1}{2} p^{2}-2 \nu p_{r}^{2}-\frac{1}{r}\right]+\frac{1}{2 r^{3} c^{3}}\left[\chi_{1}(2+2 \delta-\nu)+\chi_{2}(2-2 \delta-\nu)\right]+\frac{p_{\phi}}{c^{4} r^{2}}\left[4 \nu^{2} p_{r}^{4}+\frac{\left(3 \nu^{2}-17 \nu+2\right) p^{2}}{4 r}\right. \\
& \left.-2(\nu-1) \nu p_{r}^{2} p^{2}-\frac{\left(\nu^{2}+5 \nu-3\right) p^{4}}{8}+\frac{4-5 \nu^{2}+65 \nu}{4 r} p_{r}^{2}-\frac{\nu^{2}-9 \nu+2}{4 r^{2}}+\frac{1}{2 r^{2}}\left(X_{1}^{4} \chi_{1}^{2}+2 \nu^{2} \chi_{1} \chi_{2}+X_{2}^{4} \chi_{2}^{2}\right)\right], \\
\dot{r}_{h}= & p_{r}+\frac{p_{r}}{c^{2}}\left[\left(2 \nu-\frac{1}{2}\right) p^{2}-(2 \nu+3) \frac{1}{r}-\nu p_{r}^{2}\right]+\frac{p_{r}}{c^{4}}\left[\left(\nu^{2}-2 \nu+\frac{3}{8}\right) p^{4}+\frac{\left(\nu^{2}-55 \nu+6\right) p^{2}}{4 r}+\left(\nu-\frac{5 \nu^{2}}{2}\right) p_{r}^{2} p^{2}\right. \\
& \left.+\frac{4-\nu^{2}+39 \nu}{4 r} p_{r}^{2}+\frac{3}{2} \nu^{2} p_{r}^{4}+\frac{6-5 \nu^{2}+39 \nu}{4 r^{2}}+\frac{1}{2 r^{2}}\left(X_{1}^{4} \chi_{1}^{2}+2 \nu^{2} \chi_{1} \chi_{2}+X_{2}^{4} \chi_{2}^{2}\right)\right] .
\end{aligned}
$$

\section{Transformation for the SSC}

When calculating the spin contributions to the waveform modes, we used the source moments from Refs. [116,121] which are in terms of the covariant SSC. To transform the resulting modes to the NW SSC, we use the center-of-mass shift [100]

$$
x_{A(\mathrm{cov})}^{i} \rightarrow x_{A}^{i}+\frac{1}{2 c^{3} m_{A}}\left(\boldsymbol{v}_{A} \times \boldsymbol{S}_{A}\right)^{i}
$$

and the spin transformation [132]

$$
\boldsymbol{S}_{1}^{\mathrm{cov}}=\left(1-\frac{m_{2}}{c^{2} r}\right) \boldsymbol{S}_{1}+\frac{1}{2 c^{2}} \boldsymbol{v}_{1}\left(\boldsymbol{v}_{1} \cdot \boldsymbol{S}_{1}\right)
$$

where the spin transformation is required only for the NLO SO part of the 2PN $(2,1)$ mode.
For the scalars $\left(r, \phi, \dot{r}, \dot{\phi}, \chi_{1}, \chi_{2}\right)$, we obtain the transformations

$$
\begin{aligned}
r_{\mathrm{cov}} & =r-\frac{\nu r \dot{\phi}}{2 c^{3}}\left(\chi_{1}+\chi_{2}\right), \\
\phi_{\mathrm{cov}} & =\phi+\frac{\nu \dot{r}}{2 c^{3} r}\left(\chi_{1}+\chi_{2}\right), \\
\dot{r}_{\mathrm{cov}} & =\dot{r}+\frac{\nu \dot{r} \dot{\phi}}{2 c^{3}}\left(\chi_{1}+\chi_{2}\right), \\
\dot{\phi}_{\mathrm{cov}} & =\dot{\phi}-\frac{\nu}{2 c^{3} r^{3}}\left(1+r \dot{r}^{2}-r^{3} \dot{\phi}^{2}\right)\left(\chi_{1}+\chi_{2}\right), \\
\chi_{1}^{\mathrm{cov}} & =\chi_{1}-\frac{\chi_{1}}{2 c^{2} r}(1-\delta), \\
\chi_{2}^{\mathrm{cov}} & =\chi_{2}-\frac{\chi_{2}}{2 c^{2} r}(1+\delta) .
\end{aligned}
$$




\section{APPENDIX B: ANGULAR MOMENTUM FLUX AT LEADING-ORDER SPIN-SQUARED}

In this Appendix, we derive the angular momentum flux at leading spin-squared $\left(S_{i}^{2}\right)$ order. Here, we use unscaled variables in harmonic coordinates, but we drop the subscript $h$ to simplify the notation. We denote the orbital angular momentum $\boldsymbol{L}=\mu \boldsymbol{r} \times \boldsymbol{v}$, the relative position $\boldsymbol{r}=\boldsymbol{x}_{1}-\boldsymbol{x}_{2}$, and relative velocity $\boldsymbol{v}=d \boldsymbol{r} / d t$.

The relative acceleration $\boldsymbol{a} \equiv \boldsymbol{a}_{1}-\boldsymbol{a}_{2}$ with LO SO and SS contributions, in harmonic coordinates and the NW $\mathrm{SSC}$, is given by [100]

$$
\begin{aligned}
\boldsymbol{a}= & -M \frac{\boldsymbol{n}}{r^{2}}+\frac{1}{c^{3}}\left[3\left(2+\frac{3 m_{2}}{2 m_{1}}\right) \boldsymbol{n} \cdot\left(\boldsymbol{v} \times \boldsymbol{S}_{1}\right) \frac{\boldsymbol{n}}{r^{3}}-\left(4+\frac{3 m_{2}}{m_{1}}\right) \frac{\boldsymbol{v} \times \boldsymbol{S}_{1}}{r^{3}}+3\left(2+\frac{3 m_{2}}{2 m_{1}}\right) \frac{\dot{r}}{r^{3}} \boldsymbol{n} \times \boldsymbol{S}_{1}+1 \leftrightarrow 2\right] \\
& -\frac{3}{c^{4} \mu r^{4}}\left[\boldsymbol{n}\left(\boldsymbol{S}_{1} \cdot \boldsymbol{S}_{2}\right)+\boldsymbol{S}_{1}\left(\boldsymbol{n} \cdot \boldsymbol{S}_{2}\right)+\boldsymbol{S}_{2}\left(\boldsymbol{n} \cdot \boldsymbol{S}_{1}\right)-5 \boldsymbol{n}\left(\boldsymbol{n} \cdot \boldsymbol{S}_{1}\right)\left(\boldsymbol{n} \cdot \boldsymbol{S}_{2}\right)\right] \\
& +\frac{3}{2 c^{4} r^{4}}\left[\frac{m_{2} C_{1 \mathrm{ES}}}{m_{1} \mu}\left(-\boldsymbol{n} \boldsymbol{S}_{1}^{2}+5 \boldsymbol{n}\left(\boldsymbol{n} \cdot \boldsymbol{S}_{1}\right)^{2}-2 \boldsymbol{S}_{1}\left(\boldsymbol{n} \cdot \boldsymbol{S}_{1}\right)\right)+1 \leftrightarrow 2\right] .
\end{aligned}
$$

Since the spin evolution equations start at 1PN order, we can assume $\dot{\boldsymbol{S}}_{1}=0=\dot{\boldsymbol{S}}_{2}$ for the calculation of the LO fluxes.

The source multipole moments needed are the spin quadrupole $I^{i j}$ and the current quadrupole $J^{i j}$, which are given by $[100,110,121]$

$$
\begin{gathered}
I^{i j}=m_{1} x_{1}^{\langle i} x_{1}^{j\rangle}+\frac{3}{c^{3}} x_{1}^{\langle i}\left(\boldsymbol{v}_{1} \times \boldsymbol{S}_{1}\right)^{j\rangle}-\frac{4}{3 c^{3}} \frac{d}{d t} x_{1}^{\langle i}\left(\boldsymbol{x}_{1} \times \boldsymbol{S}_{1}\right)^{j\rangle} \\
-\frac{C_{1 \mathrm{ES}}{ }^{4}}{c^{4} m_{1}} S_{1}^{\langle i} S_{1}^{j\rangle}+1 \leftrightarrow 2, \\
J^{i j}=m_{1} x_{1}^{\langle i}\left(\boldsymbol{x}_{1} \times \boldsymbol{v}_{1}\right)^{j\rangle}+\frac{3}{2 c} x_{1}^{\langle i} S_{1}^{j\rangle}+1 \leftrightarrow 2,
\end{gathered}
$$

where the indices in angle brackets denote a symmetric trace-free part.

To transform from the coordinates of the two bodies $x_{1}^{i}$ and $x_{2}^{i}$ to the center-of-mass relative coordinates $x^{i}=$ $x_{1}^{i}-x_{2}^{i}$, we use [133]

$$
x_{1}^{i}=\frac{m_{2}}{M} x^{i}+\delta x^{i}, \quad x_{2}^{i}=-\frac{m_{1}}{M} x^{i}+\delta x^{i},
$$

where

$$
\delta x^{i}=-\frac{\nu}{2 c^{3}}\left[\frac{\left(\boldsymbol{v} \times \boldsymbol{S}_{1}\right)^{i}}{m_{1}}-\frac{\left(\boldsymbol{v} \times \boldsymbol{S}_{2}\right)^{i}}{m_{2}}\right] .
$$

The energy and angular momentum fluxes in terms of the multipole moments, to the order needed for the LO fluxes, are then calculated from $[100,134]$

$$
\Phi_{E}=\frac{1}{5} I_{i j}^{(3)} I_{i j}^{(3)}+\frac{16}{45 c^{2}} J_{i j}^{(3)} J_{i j}^{(3)}
$$

$$
\Phi_{J}^{i}=\frac{2}{5} \epsilon_{i j k} I_{j l}^{(2)} I_{k l}^{(3)}+\frac{32}{45 c^{2}} \epsilon_{i j k} J_{j l}^{(2)} J_{k l}^{(3)} .
$$

This yields the LO SO and $\mathrm{S}_{1} \mathrm{~S}_{2}$ fluxes derived in Refs. $[92,93,100]$, in addition to the $S_{i}^{2}$ energy flux from Ref. [110]. For the $S_{i}^{2}$ angular momentum flux, we obtain

$$
\begin{aligned}
\Phi_{J}^{S_{i}^{2}}= & \frac{2 m_{2}^{2}}{5 c^{4} r^{5}}\left[\frac{\boldsymbol{L}}{\mu r} \boldsymbol{S}_{1}^{2}-\boldsymbol{n} \cdot \boldsymbol{S}_{1}\left(\boldsymbol{v} \times \boldsymbol{S}_{1}\right)+\boldsymbol{v} \cdot \boldsymbol{S}_{1}\left(\boldsymbol{n} \times \boldsymbol{S}_{1}\right)\right] \\
& +\frac{2 m_{2}^{2} C_{1 \mathrm{ES}}}{5 c^{4} M r^{4}}\left[\frac{\boldsymbol{L}}{\mu r} \boldsymbol{S}_{1}^{2}\left(-30 \dot{r}^{2}+12 v^{2}+24 \frac{M}{r}\right)+\frac{\boldsymbol{L}}{\mu r}\left(\boldsymbol{n} \cdot \boldsymbol{S}_{1}\right)^{2}\left(210 \dot{r}^{2}-60 v^{2}-90 \frac{M}{r}\right)\right. \\
& +\boldsymbol{v} \times \boldsymbol{S}_{1}\left(\boldsymbol{n} \cdot \boldsymbol{S}_{1}\right)\left(30 \dot{r}^{2}-18 v^{2}-12 \frac{M}{r}\right)+6 \boldsymbol{n} \times \boldsymbol{S}_{1}\left(\boldsymbol{v} \cdot \boldsymbol{S}_{1}-\dot{r} \boldsymbol{n} \cdot \boldsymbol{S}_{1}\right) \frac{M}{r} \\
& \left.-90 \frac{\boldsymbol{L}}{\mu r} \dot{r}\left(\boldsymbol{n} \cdot \boldsymbol{S}_{1}\right)\left(\boldsymbol{v} \cdot \boldsymbol{S}_{1}\right)+6 \frac{\boldsymbol{L}}{\mu r}\left(\boldsymbol{v} \cdot \boldsymbol{S}_{1}\right)^{2}-6 \dot{r}\left(\boldsymbol{v} \cdot \boldsymbol{S}_{1}\right) \boldsymbol{v} \times \boldsymbol{S}_{1}\right]+1 \leftrightarrow 2 .
\end{aligned}
$$


This is in agreement with the recent results of Ref. [135], although our expression appears simpler because of using the individual spins $S_{i}$ and masses $m_{i}$, instead of different combinations of them.

\section{APPENDIX C: ALIGNED-SPIN CONTRIBUTIONS TO THE MODES IN HARMONIC COORDINATES}

The modes calculated from the source moments of Refs. $[116,121]$ in harmonic coordinates and using the covariant SSC have the following spin contributions:

$$
\begin{aligned}
& \hat{H}_{\text {spin }}^{20}=\frac{\dot{\phi}}{\sqrt{6} c^{3} r}\left[\chi_{1}(1+\delta+\nu)+\chi_{2}(1-\delta+\nu)\right]-\frac{\sqrt{3}}{2 \sqrt{2} c^{4} r^{3}}\left[C_{1 \mathrm{ES}^{2}} X_{1}^{2} \chi_{1}^{2}+2 \nu \chi_{1} \chi_{2}+C_{2 \mathrm{ES}^{2}} X_{2}^{2} \chi_{2}^{2}\right], \\
& \hat{H}_{\text {spin }}^{21}=-\frac{i}{4 r^{2}}\left[(1+\delta) \chi_{1}+(\delta-1) \chi_{2}\right]+\frac{i}{168 c^{4} r^{3}}\left\{\chi _ { 1 } \left[154+22 \delta(\nu+7)+34 \nu+4 r^{3} \dot{\phi}^{2}(4 \nu \delta-21 \delta+66 \nu-21)\right.\right. \\
& \left.-2 i \dot{r} r^{2} \dot{\phi}(13 \nu \delta+147 \delta-83 \nu+147)+\dot{r}^{2} r(-60 \delta \nu+105 \delta-52 \nu+105)\right]+\chi_{2}[-154+22 \delta(\nu+7)-34 \nu \\
& \left.\left.+4 r^{3} \dot{\phi}^{2}(4 \nu \delta-21 \delta-66 \nu+21)-2 i \dot{r} r^{2} \dot{\phi}(13 \nu \delta+147 \delta+83 \nu-147)+\dot{r}^{2} r(-60 \delta \nu+105 \delta+52 \nu-105)\right]\right\}, \\
& \hat{H}_{\text {spin }}^{22}=-\frac{1}{6 c^{3} r^{2}}\left\{\chi_{1}[r \dot{\phi}(3 \delta-5 \nu+3)+i \dot{r}(3 \delta-8 \nu+3)]+\chi_{2}[r \dot{\phi}(-3 \delta-5 \nu+3)-i \dot{r}(3 \delta+8 \nu-3)]\right\} \\
& +\frac{3}{4 c^{4} r^{3}}\left[C_{1 \mathrm{ES}^{2}} \chi_{1}^{2} X_{1}^{2}+C_{2 \mathrm{ES}^{2}} \chi_{2}^{2} X_{2}^{2}+2 \nu \chi_{2} \chi_{1}\right] \\
& \hat{H}_{\text {spin }}^{30}=-\frac{i \nu \dot{r}}{\sqrt{42} c^{3} r^{2}}\left(\chi_{1}+\chi_{2}\right) \text {, } \\
& \hat{H}_{\text {spin }}^{31}=\frac{i}{48 \sqrt{14} c^{4} r^{3}}\left\{\chi _ { 1 } \left[-4+20 \delta \nu-4 \delta+20 \nu+r^{3} \dot{\phi}^{2}(-31 \delta \nu-24 \delta+87 \nu-24)+i \dot{r} r^{2} \dot{\phi}(-70 \delta \nu-12 \delta+62 \nu-12)\right.\right. \\
& \left.+\dot{r}^{2} r(-30 \delta \nu+12 \delta-50 \nu+12)\right]+\chi_{2}\left[4+20 \delta \nu-4 \delta-20 \nu+r^{3} \dot{\phi}^{2}(-31 \delta \nu-24 \delta-87 \nu+24)\right. \\
& \left.\left.+i \dot{r} r^{2} \dot{\phi}(-70 \delta \nu-12 \delta-62 \nu+12)+\dot{r}^{2} r(-30 \delta \nu+12 \delta+50 \nu-12)\right]\right\}
\end{aligned}
$$

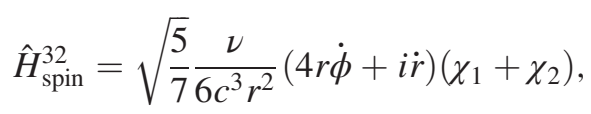

$$
\begin{aligned}
& \hat{H}_{\mathrm{spin}}^{33}=\sqrt{\frac{5}{42}} \frac{i}{16 c^{4} r^{3}}\left\{\chi _ { 1 } \left[4-20 \delta \nu+4 \delta-20 \nu+r^{3} \dot{\phi}^{2}(-33 \delta \nu+24 \delta-119 \nu+24)+i \dot{r} r^{2} \dot{\phi}(-78 \delta \nu+36 \delta-154 \nu+36)\right.\right. \\
& \left.+\dot{r}^{2} r(30 \delta \nu-12 \delta+50 \nu-12)\right]+\chi_{2}\left[-4-20 \delta \nu+4 \delta+20 \nu+r^{3} \dot{\phi}^{2}(-33 \delta \nu+24 \delta+119 \nu-24)\right. \\
& \left.\left.+i \dot{r} r^{2} \dot{\phi}(-78 \delta \nu+36 \delta+154 \nu-36)+\dot{r}^{2} r(30 \delta \nu-12 \delta-50 \nu+12)\right]\right\}, \\
& \hat{H}_{\mathrm{spin}}^{41}=-i \sqrt{\frac{5}{2}} \frac{\nu}{336 c^{4} r^{3}}\left(11 r^{3} \dot{\phi}^{2}-10 i \dot{r} r^{2} \dot{\phi}+6 \dot{r}^{2} r-12\right)\left[(\delta-1) \chi_{1}+(\delta+1) \chi_{2}\right], \\
& \hat{H}_{\text {spin }}^{43}=\sqrt{\frac{5}{14}} \frac{\nu}{48 c^{4} r^{3}}\left(-23 i r^{3} \dot{\phi}^{2}+10 \dot{r} r^{2} \dot{\phi}+2 i \dot{r}^{2} r-4 i\right)\left[(\delta-1) \chi_{1}+(\delta+1) \chi_{2}\right] .
\end{aligned}
$$

\section{APPENDIX D: KEPLERIAN PARAMETRIZATION}

This Appendix provides expressions for some orbital quantities in the Keplerian parametrization that are needed for calculating the initial conditions, and the tail part of the RR force and waveform modes.

In the Keplerian parametrization,

$$
r=\frac{1}{u_{p}(1+e \cos \chi)}
$$

where $u_{p}$ is the inverse semilatus rectum and $\chi$ is the relativistic anomaly. Inverting the Hamiltonian at the turning points $r_{ \pm}=1 /\left(u_{p}(1 \pm e)\right)$ and solving for the energy and angular momentum to $2 \mathrm{PN}$ order yields 


$$
\begin{aligned}
& E=\frac{1}{2}\left(e^{2}-1\right) u_{p}-\frac{u_{p}^{2}}{8 c^{2}}\left(e^{2}-1\right)^{2}(\nu-3) \\
& +\frac{u_{p}^{3}}{16 c^{4}}\left(e^{2}-1\right)^{2}\left[e^{2}\left(\nu^{2}-3 \nu+5\right)-\nu^{2}-5 \nu+27\right] \\
& +\frac{\left(1-e^{2}\right)^{2} u_{p}^{5 / 2}}{4 c^{4}}\left[\chi_{1}(\nu-2 \delta-2)+\chi_{2}(\nu+2 \delta-2)\right] \\
& +\frac{\left(1-e^{2}\right)^{2} u_{p}^{3}}{4 c^{4}}\left[\chi_{1}^{2}\left(C_{1 \mathrm{ES}^{2}} X_{1}^{2}+X_{1}^{4}\right)\right. \\
& \left.+2 \nu(1+\nu) \chi_{2} \chi_{1}+\chi_{2}^{2}\left(C_{2 \mathrm{ES}^{2}} X_{2}^{2}+X_{2}^{4}\right)\right], \\
& p_{\phi}=\frac{1}{\sqrt{u_{p}}}+\frac{\sqrt{u_{p}}}{2 c^{2}}\left(e^{2}+3\right)+\frac{u_{p}^{3 / 2}}{8 c^{4}}\left(e^{2}+3\right)\left(3 e^{2}-4 \nu+9\right) \\
& +\frac{1}{4}\left(e^{2}+3\right) u_{p}\left[\chi_{1}(-2 \delta+\nu-2)+\chi_{2}(2 \delta+\nu-2)\right] \\
& +\frac{\left(e^{2}+3\right) u_{p}^{3 / 2}}{4 c^{4}}\left[\chi_{1}^{2}\left(C_{1 \mathrm{ES}^{2}} X_{1}^{2}+X_{1}^{4}\right)\right. \\
& \left.+\chi_{2}^{2}\left(C_{2 \mathrm{ES}^{2}} X_{2}^{2}+X_{2}^{4}\right)\right] \\
& +\frac{\nu u_{p}^{3 / 2} \chi_{1} \chi_{2}}{2 c^{4}}\left[e^{2}(3 \nu+1)+\nu+3\right] \text {. }
\end{aligned}
$$

Inverting the Hamiltonian to obtain $p_{r}\left(E, p_{\phi}\right)$, and plugging $E\left(e, u_{p}\right)$ and $p_{\phi}\left(e, u_{p}\right)$, yields

$p_{r}=e \sqrt{u_{p}} \sin \chi+\frac{e u_{p}^{3 / 2}}{2 c^{2}} \sin \chi\left(e^{2}+2 e \cos \chi+1\right)+\cdots$

The radial and azimuthal periods are given, respectively, by

$$
\begin{aligned}
T_{r} & =\oint d t=\oint\left(\frac{\partial H}{\partial p_{r}}\right)^{-1} d r=2 \int_{0}^{\pi}\left(\frac{\partial H}{\partial p_{r}}\right)^{-1} \frac{d r}{d \chi} d \chi \\
& =\frac{2 \pi}{\left(u_{p}-e^{2} u_{p}\right)^{3 / 2}}-\frac{\pi(\nu-6)}{c^{2} \sqrt{u_{p}-e^{2} u_{p}}}+\cdots, \\
T_{\phi} & =\oint \dot{\phi} d t=\oint \frac{\partial H}{\partial p_{\phi}} d t=2 \pi+\frac{6 \pi u_{p}}{c^{2}}+\cdots .
\end{aligned}
$$

Inverting $p_{\phi}\left(u_{p}, e\right)$, we obtain $u_{p}\left(p_{\phi}, e\right)$

$$
\begin{aligned}
u_{p}\left(p_{\phi}, e\right)= & \frac{1}{p_{\phi}^{2}}+\frac{\left(e^{2}+3\right)}{c^{2} p_{\phi}^{4}}+\frac{\left(e^{2}+3\right)\left(2 e^{2}-\nu+6\right)}{c^{4} p_{\phi}^{6}} \\
& +\frac{3+e^{2}}{2 p_{\phi}^{5} c^{3}}\left[(\nu-2 \delta-2) \chi_{1}+(\nu+2 \delta-2) \chi_{2}\right] \\
& +\frac{1}{2 p_{\phi}^{6} c^{4}}\left\{\nu \chi_{1} \chi_{2}\left[e^{2}(3 \nu+1)+\nu+3\right]\right. \\
& +\chi_{1}^{2}\left[C_{1 \mathrm{ES}^{2}}\left(e^{2}+3\right) X_{1}^{2}+\left(3 e^{2}+1\right) X_{1}^{4}\right] \\
& +1 \leftrightarrow 2\} .
\end{aligned}
$$

The associated frequencies are

$$
\omega_{r}=\frac{2 \pi}{T_{r}}, \quad \omega_{\phi}=\frac{T_{\phi}}{T_{r}}
$$

The dimensionless frequency variable $x \equiv \omega_{\phi}^{2 / 3}$ to $2 \mathrm{PN}$ order is given by

$$
\begin{aligned}
x= & u_{p}-e^{2} u_{p}+\frac{u_{p}^{2}}{3 c^{2}}\left(e^{2}-1\right)\left(e^{2}(\nu-6)-\nu\right)-\frac{u_{p}^{3}}{36 c^{4}}\left(e^{2}-1\right)\left\{e^{4}\left(8 \nu^{2}-33 \nu+180\right)+8 \nu^{2}\right. \\
& \left.-2 e^{2}\left[3\left(12 \sqrt{1-e^{2}}+5\right) \nu-90 \sqrt{1-e^{2}}+8 \nu^{2}+27\right]+9\left(8 \sqrt{1-e^{2}}-13\right) \nu-180\left(\sqrt{1-e^{2}}-1\right)\right\} \\
& +\frac{u_{p}^{5 / 2}}{6 c^{4}}\left(e^{2}-1\right)\left(3 e^{2}+1\right)\left[\chi_{1}(2+2 \delta-\nu)+1 \leftrightarrow 2\right] \\
& -\frac{u_{p}^{3}}{2 c^{4}}\left\{\chi_{1}^{2}\left[C_{1 \mathrm{ES}^{2}}\left(e^{4}-1\right) X_{1}^{2}+\left(e^{2}-1\right)^{2} X_{1}^{4}\right]+\nu \chi_{1} \chi_{2}\left[e^{4}(\nu+1)-2 e^{2} \nu+\nu-1\right]+1 \leftrightarrow 2\right\},
\end{aligned}
$$

which can be inverted to obtain $u_{p}(x, e)$, the $1 \mathrm{PN}$ part of which reads

$$
u_{p}=-\frac{x}{e^{2}-1}+\frac{x^{2}\left[e^{2}(\nu-6)-\nu\right]}{3 c^{2}\left(e^{2}-1\right)^{2}}+\cdots
$$

\section{APPENDIX E: $\dot{p}_{r}$ IN TORTOISE COORDINATES}

The tortoise coordinate $r_{*}$ is defined by $[69,112]$

$$
\frac{d r_{*}}{d r}=\frac{\sqrt{D(r)}}{A(r)} \equiv \frac{1}{\xi(r)},
$$


where $A(r)$ and $D(r)$ are the metric potentials

$$
d s_{\mathrm{eff}}^{2}=-A(r) d t^{2}+\frac{D(r)}{A(r)} d r^{2}+r^{2} d \Omega^{2} .
$$

The conjugate momentum to $r_{*}$ is denoted $p_{r_{*}}$, and invariance of the action gives the relation

$$
p_{r_{*}}=p_{r} \xi(r) \text {. }
$$

The Hamiltonian and EOMs used in SEOBNRv4 [see Eqs. (10) of Ref. [136]] are expressed in terms of the variables $\left(r, p_{r_{*}}, \phi, p_{\phi}\right)$. However, the RR force we derived in Sec. II is expressed in terms of $\left(r, p_{r}, \dot{p}_{r}\right)$. We use Eq. (E3) to replace $p_{r}$ with $p_{r_{*}}$, and to obtain a relation between $\dot{p}_{r}$ and the derivatives of $H_{\mathrm{EOB}}\left(r, p_{r_{*}}, p_{\phi}\right)$. We use the following relations:

$$
\begin{aligned}
d H & =\left(\frac{\partial H}{\partial r}\right)_{p_{r_{*}}} d r+\left(\frac{\partial H}{\partial p_{r_{*}}}\right)_{r} d p_{r_{*}}+\frac{\partial H}{\partial p_{\phi}} d p_{\phi} \\
& =\left(\frac{\partial H}{\partial r}\right)_{p_{r}} d r+\left(\frac{\partial H}{\partial p_{r}}\right)_{r} d p_{r}+\frac{\partial H}{\partial p_{\phi}} d p_{\phi},
\end{aligned}
$$

$$
d p_{r_{*}}=\left(\frac{\partial p_{r_{*}}}{\partial r}\right)_{p_{r}} d r+\left(\frac{\partial p_{r_{*}}}{\partial r}\right)_{r} d p_{r}
$$

leading to

$$
\left(\frac{\partial H}{\partial r}\right)_{p_{r}}=\left(\frac{\partial H}{\partial r}\right)_{p_{r_{*}}}+\left(\frac{\partial H}{\partial p_{r_{*}}}\right)_{r}\left(\frac{\partial p_{r_{*}}}{\partial r}\right)_{p_{r}},
$$

where

$$
\left(\frac{\partial p_{r_{*}}}{\partial r}\right)_{p_{r}}=p_{r} \frac{d \xi(r)}{d r}
$$

Hence,

$$
\begin{aligned}
\dot{p}_{r} & =-\left(\frac{\partial H}{\partial r}\right)_{p_{r}} \\
& =-\left[\left(\frac{\partial H}{\partial r}\right)_{p_{r_{*}}}+\left(\frac{\partial H}{\partial p_{r_{*}}}\right)_{r} \frac{p_{r_{*}}}{\xi(r)} \frac{d \xi(r)}{d r}\right] .
\end{aligned}
$$

[1] R. Abbott et al. (LIGO Scientific and Virgo Collaborations), GWTC-2: Compact binary coalescences observed by LIGO and Virgo during the first half of the third observing run, Phys. Rev. X 11, 021053 (2021).

[2] B. P. Abbott et al. (LIGO Scientific and Virgo Collaborations), GWTC-1: A Gravitational-Wave Transient Catalog of Compact Binary Mergers Observed by LIGO and Virgo during the First and Second Observing Runs, Phys. Rev. X 9, 031040 (2019).

[3] B. P. Abbott et al. (LIGO Scientific and Virgo Collaborations), Binary black hole population properties inferred from the first and second observing runs of advanced LIGO and Advanced Virgo, Astrophys. J. Lett. 882, L24 (2019).

[4] R. Abbott et al. (LIGO Scientific and Virgo Collaborations), Population properties of compact objects from the second LIGO-Virgo gravitational-wave transient catalog, Astrophys. J. Lett. 913, L7 (2021).

[5] B. Abbott et al. (LIGO Scientific and Virgo Collaborations), Search for eccentric binary black hole mergers with advanced LIGO and Advanced Virgo during their first and second observing runs, Astrophys. J. 883, 149 (2019).

[6] I. M. Romero-Shaw, P. D. Lasky, and E. Thrane, Searching for eccentricity: Signatures of dynamical formation in the first gravitational-wave transient catalogue of LIGO and Virgo, Mon. Not. R. Astron. Soc. 490, 5210 (2019).

[7] A. H. Nitz, A. Lenon, and D. A. Brown, Search for Eccentric binary neutron star mergers in the first and second observing runs of advanced LIGO, Astrophys. J. 890, 1 (2019).

[8] I. M. Romero-Shaw, P. D. Lasky, E. Thrane, and J.C. Bustillo, GW190521: Orbital eccentricity and signatures of dynamical formation in a binary black hole merger signal, Astrophys. J. Lett. 903, L5 (2020).

[9] P. Peters, Gravitational radiation and the motion of two point masses, Phys. Rev. 136, B1224 (1964).

[10] I. Hinder, B. Vaishnav, F. Herrmann, D. Shoemaker, and P. Laguna, Universality and final spin in eccentric binary black hole inspirals, Phys. Rev. D 77, 081502 (2008).

[11] J. Samsing and E. Ramirez-Ruiz, On the assembly rate of highly eccentric binary black hole mergers, Astrophys. J. Lett. 840, L14 (2017).

[12] J. Samsing, M. MacLeod, and E. Ramirez-Ruiz, The formation of eccentric compact binary inspirals and the role of gravitational wave emission in binary-single stellar encounters, Astrophys. J. 784, 71 (2014).

[13] J. Samsing, Eccentric black hole mergers forming in globular clusters, Phys. Rev. D 97, 103014 (2018).

[14] C. L. Rodriguez, P. Amaro-Seoane, S. Chatterjee, and F. A. Rasio, Post-Newtonian Dynamics in Dense Star Clusters: Highly-Eccentric, Highly-Spinning, and Repeated Binary Black Hole Mergers, Phys. Rev. Lett. 120, 151101 (2018).

[15] M. Zevin, J. Samsing, C. Rodriguez, C.-J. Haster, and E. Ramirez-Ruiz, Eccentric black hole mergers in dense star clusters: The role of binary-binary encounters, Astrophys. J. 871, 91 (2019). 
[16] L. Gondán and B. Kocsis, High eccentricities and high masses characterize gravitational-wave captures in galactic nuclei as seen by earth-based detectors, arXiv:2011.02507.

[17] F. Antonini and H. B. Perets, Secular evolution of compact binaries near massive black holes: Gravitational wave sources and other exotica, Astrophys. J. 757, 27 (2012).

[18] F. Antonini, S. Chatterjee, C. L. Rodriguez, M. Morscher, B. Pattabiraman, V. Kalogera, and F. A. Rasio, Black hole mergers and blue stragglers from hierarchical triples formed in globular clusters, Astrophys. J. 816, 65 (2016).

[19] J. H. VanLandingham, M. Miller, D. P. Hamilton, and D. C. Richardson, The role of the Kozai-lidov mechanism in black hole binary mergers in galactic centers, Astrophys. J. 828, 77 (2016).

[20] R. Abbott et al. (LIGO Scientific and Virgo Collaborations), GW190521: A Binary Black Hole Merger with a Total Mass of $150 M_{\odot}$, Phys. Rev. Lett. 125, 101102 (2020).

[21] B. P. Abbott et al. (KAGRA, LIGO Scientific, and Virgo Collaborations), Prospects for observing and localizing gravitational-wave transients with Advanced LIGO, Advanced Virgo and KAGRA, Living Rev. Relativity 23, 3 (2020).

[22] M. Favata, Systematic Parameter Errors in Inspiraling Neutron Star Binaries, Phys. Rev. Lett. 112, 101101 (2014).

[23] A. Sesana, Self consistent model for the evolution of eccentric massive black hole binaries in stellar environments: Implications for gravitational wave observations, Astrophys. J. 719, 851 (2010).

[24] K. Breivik, C. L. Rodriguez, S. L. Larson, V. Kalogera, and F. A. Rasio, Distinguishing Between Formation Channels for Binary Black Holes with LISA, Astrophys. J. Lett. 830, L18 (2016).

[25] B. Willems, V. Kalogera, A. Vecchio, N. Ivanova, F. Rasio, J. Fregeau, and K. Belczynski, Eccentric double white dwarfs as LISA sources in globular clusters, Astrophys. J. Lett. 665, L59 (2007).

[26] J. Samsing and D. J. D’Orazio, Black hole mergers from globular clusters observable by LISA I: Eccentric sources originating from relativistic $N$-body dynamics, Mon. Not. R. Astron. Soc. 481, 5445 (2018).

[27] V. Cardoso, C. F. Macedo, and R. Vicente, Eccentricity evolution of compact binaries and applications to gravitational-wave physics, Phys. Rev. D 103, 023015 (2021).

[28] R.-M. Memmesheimer, A. Gopakumar, and G. Schaefer, Third post-Newtonian accurate generalized quasiKeplerian parametrization for compact binaries in eccentric orbits, Phys. Rev. D 70, 104011 (2004).

[29] Y. Boetzel, A. Susobhanan, A. Gopakumar, A. Klein, and P. Jetzer, Solving post-Newtonian accurate Kepler Equation, Phys. Rev. D 96, 044011 (2017).

[30] N. Loutrel and N. Yunes, Eccentric Gravitational Wave Bursts in the Post-Newtonian Formalism, Classical Quant. Grav. 34, 135011 (2017).

[31] S. Tanay, M. Haney, and A. Gopakumar, Frequency and time domain inspiral templates for comparable mass compact binaries in eccentric orbits, Phys. Rev. D 93, 064031 (2016).
[32] N. Yunes, K. Arun, E. Berti, and C. M. Will, Post-circular expansion of eccentric binary inspirals: Fourier-domain waveforms in the stationary phase approximation, Phys. Rev. D 80, 084001 (2009); Erratum, Phys. Rev. D 89, 109901 (2014).

[33] E. A. Huerta, P. Kumar, S. T. McWilliams, R. O’Shaughnessy, and N. Yunes, Accurate and efficient waveforms for compact binaries on eccentric orbits, Phys. Rev. D 90, 084016 (2014).

[34] S. Tiwari, G. Achamveedu, M. Haney, and P. Hemantakumar, Ready-to-use Fourier domain templates for compact binaries inspiraling along moderately eccentric orbits, Phys. Rev. D 99, 124008 (2019).

[35] A. Klein, Y. Boetzel, A. Gopakumar, P. Jetzer, and L. de Vittori, Fourier domain gravitational waveforms for precessing eccentric binaries, Phys. Rev. D 98, 104043 (2018).

[36] T. Damour, A. Gopakumar, and B. R. Iyer, Phasing of gravitational waves from inspiralling eccentric binaries, Phys. Rev. D 70, 064028 (2004).

[37] C. Konigsdorffer and A. Gopakumar, Phasing of gravitational waves from inspiralling eccentric binaries at the third-and-a-half post-Newtonian order, Phys. Rev. D 73, 124012 (2006).

[38] B. Moore, M. Favata, K. Arun, and C. K. Mishra, Gravitational-wave phasing for low-eccentricity inspiralling compact binaries to 3PN order, Phys. Rev. D 93, 124061 (2016).

[39] I. Hinder, L. E. Kidder, and H. P. Pfeiffer, Eccentric binary black hole inspiral-merger-ringdown gravitational waveform model from numerical relativity and post-Newtonian theory, Phys. Rev. D 98, 044015 (2018).

[40] E. Huerta et al., Complete waveform model for compact binaries on eccentric orbits, Phys. Rev. D 95, 024038 (2017).

[41] A. Ramos-Buades, S. Husa, G. Pratten, H. Estellés, C. García-Quirós, M. Mateu-Lucena, M. Colleoni, and R. Jaume, First survey of spinning eccentric black hole mergers: Numerical relativity simulations, hybrid waveforms, and parameter estimation, Phys. Rev. D 101, 083015 (2020).

[42] E. A. Huerta et al., Physics of eccentric binary black hole mergers: A numerical relativity perspective, Phys. Rev. D 100, 064003 (2019).

[43] M. Boyle et al., The SXS Collaboration catalog of binary black hole simulations, Classical Quant. Grav. 36, 195006 (2019).

[44] T. Islam, V. Varma, J. Lodman, S. E. Field, G. Khanna, M. A. Scheel, H. P. Pfeiffer, D. Gerosa, and L. E. Kidder, Eccentric binary black hole surrogate models for the gravitational waveform and remnant properties: Comparable mass, nonspinning case, Phys. Rev. D 103, 064022 (2021).

[45] A. Buonanno and T. Damour, Effective one-body approach to general relativistic two-body dynamics, Phys. Rev. D 59, 084006 (1999).

[46] A. Buonanno and T. Damour, Transition from inspiral to plunge in binary black hole coalescences, Phys. Rev. D 62 , 064015 (2000). 
[47] T. Damour, P. Jaranowski, and G. Schäfer, Fourth postNewtonian effective one-body dynamics, Phys. Rev. D 91, 084024 (2015).

[48] T. Damour, Coalescence of two spinning black holes: An effective one-body approach, Phys. Rev. D 64, 124013 (2001).

[49] T. Damour, P. Jaranowski, and G. Schaefer, Effective one body approach to the dynamics of two spinning black holes with next-to-leading order spin-orbit coupling, Phys. Rev. D 78, 024009 (2008).

[50] E. Barausse and A. Buonanno, An Improved effective-onebody Hamiltonian for spinning black-hole binaries, Phys. Rev. D 81, 084024 (2010).

[51] E. Barausse and A. Buonanno, Extending the effectiveone-body Hamiltonian of black-hole binaries to include next-to-next-to-leading spin-orbit couplings, Phys. Rev. D 84, 104027 (2011).

[52] A. Nagar, Effective one body Hamiltonian of two spinning black-holes with next-to-next-to-leading order spin-orbit coupling, Phys. Rev. D 84, 084028 (2011); Erratum, 88, 089901 (2013).

[53] S. Balmelli and P. Jetzer, Effective-one-body Hamiltonian with next-to-leading order spin-spin coupling for two nonprecessing black holes with aligned spins, Phys. Rev. D 87, 124036 (2013); Erratum, Phys. Rev. D 90, 089905 (2014).

[54] T. Damour and A. Nagar, New effective-one-body description of coalescing nonprecessing spinning black-hole binaries, Phys. Rev. D 90, 044018 (2014).

[55] S. Balmelli and T. Damour, New effective-one-body Hamiltonian with next-to-leading order spin-spin coupling, Phys. Rev. D 92, 124022 (2015).

[56] M. Khalil, J. Steinhoff, J. Vines, and A. Buonanno, Fourth post-Newtonian effective-one-body Hamiltonians with generic spins, Phys. Rev. D 101, 104034 (2020).

[57] T. Damour and A. Nagar, Effective one body description of tidal effects in inspiralling compact binaries, Phys. Rev. D 81, 084016 (2010).

[58] D. Bini, T. Damour, and G. Faye, Effective action approach to higher-order relativistic tidal interactions in binary systems and their effective one body description, Phys. Rev. D 85, 124034 (2012).

[59] J. Steinhoff, T. Hinderer, A. Buonanno, and A. Taracchini, Dynamical tides in general relativity: Effective action and effective-one-body Hamiltonian, Phys. Rev. D 94, 104028 (2016).

[60] T. Hinderer et al., Effects of Neutron-Star Dynamic Tides On Gravitational Waveforms Within the Effective-OneBody Approach, Phys. Rev. Lett. 116, 181101 (2016).

[61] N. Yunes, A. Buonanno, S. A. Hughes, Y. Pan, E. Barausse, M. Miller, and W. Throwe, Extreme mass-ratio inspirals in the effective-one-body approach: Quasi-circular, equatorial orbits around a spinning black hole, Phys. Rev. D 83, 044044 (2011); Erratum, 88, 109904 (2013).

[62] T. Damour, Gravitational self force in a schwarzschild background and the effective one body formalism, Phys. Rev. D 81, 024017 (2010).

[63] E. Barausse, A. Buonanno, and A. Le Tiec, The complete non-spinning effective-one-body metric at linear order in the mass ratio, Phys. Rev. D 85, 064010 (2012).
[64] S. Akcay, L. Barack, T. Damour, and N. Sago, Gravitational self-force and the effective-one-body formalism between the innermost stable circular orbit and the light ring, Phys. Rev. D 86, 104041 (2012).

[65] A. Antonelli, M. van de Meent, A. Buonanno, J. Steinhoff, and J. Vines, Quasicircular inspirals and plunges from nonspinning effective-one-body Hamiltonians with gravitational self-force information, Phys. Rev. D 101, 024024 (2020).

[66] T. Damour, Gravitational scattering, post-Minkowskian approximation and effective one-body theory, Phys. Rev. D 94, 104015 (2016).

[67] T. Damour, High-energy gravitational scattering and the general relativistic two-body problem, Phys. Rev. D 97, 044038 (2018).

[68] A. Antonelli, A. Buonanno, J. Steinhoff, M. van de Meent, and J. Vines, Energetics of two-body Hamiltonians in post-Minkowskian gravity, Phys. Rev. D 99, 104004 (2019).

[69] Y. Pan, A. Buonanno, L. T. Buchman, T. Chu, L. E. Kidder, H. P. Pfeiffer, and M. A. Scheel, Effective-one-body waveforms calibrated to numerical relativity simulations: Coalescence of non-precessing, spinning, equal-mass black holes, Phys. Rev. D 81, 084041 (2010).

[70] Y. Pan, A. Buonanno, R. Fujita, E. Racine, and H. Tagoshi, Post-Newtonian factorized multipolar waveforms for spinning, non-precessing black-hole binaries, Phys. Rev. D 83, 064003 (2011); Erratum, Phys. Rev. D 87, 109901 (2013).

[71] Y. Pan, A. Buonanno, A. Taracchini, L. E. Kidder, A. H. Mroué, H. P. Pfeiffer, M. A. Scheel, and B. Szilágyi, Inspiral-merger-ringdown waveforms of spinning, precessing black-hole binaries in the effective-one-body formalism, Phys. Rev. D 89, 084006 (2014).

[72] A. Taracchini, Y. Pan, A. Buonanno, E. Barausse, M. Boyle, T. Chu, G. Lovelace, H. P. Pfeiffer, and M. A. Scheel, Prototype effective-one-body model for nonprecessing spinning inspiral-merger-ringdown waveforms, Phys. Rev. D 86, 024011 (2012).

[73] A. Taracchini et al., Effective-one-body model for blackhole binaries with generic mass ratios and spins, Phys. Rev. D 89, 061502 (2014).

[74] A. Bohé et al., Improved effective-one-body model of spinning, nonprecessing binary black holes for the era of gravitational-wave astrophysics with advanced detectors, Phys. Rev. D 95, 044028 (2017).

[75] S. Babak, A. Taracchini, and A. Buonanno, Validating the effective-one-body model of spinning, precessing binary black holes against numerical relativity, Phys. Rev. D 95, 024010 (2017).

[76] A. Nagar et al., Time-domain effective-one-body gravitational waveforms for coalescing compact binaries with nonprecessing spins, tides and self-spin effects, Phys. Rev. D 98, 104052 (2018).

[77] S. Ossokine et al., Multipolar effective-one-body waveforms for precessing binary black holes: Construction and validation, Phys. Rev. D 102, 044055 (2020).

[78] D. Bini and T. Damour, Gravitational radiation reaction along general orbits in the effective one-body formalism, Phys. Rev. D 86, 124012 (2012). 
[79] T. Hinderer and S. Babak, Foundations of an effective-onebody model for coalescing binaries on eccentric orbits, Phys. Rev. D 96, 104048 (2017).

[80] Z. Cao and W.-B. Han, Waveform model for an eccentric binary black hole based on the effective-one-body-numericalrelativity formalism, Phys. Rev. D 96, 044028 (2017).

[81] X. Liu, Z. Cao, and L. Shao, Validating the effectiveone-body numerical-relativity waveform models for spinaligned binary black holes along eccentric orbits, Phys. Rev. D 101, 044049 (2020).

[82] X. Liu, Z. Cao, and Z.-H. Zhu, A higher-multipole gravitational waveform model for an eccentric binary black holes based on the effective-one-body-numericalrelativity formalism, arXiv:2102.08614.

[83] R. Cotesta, A. Buonanno, A. Bohé, A. Taracchini, I. Hinder, and S. Ossokine, Enriching the Symphony of Gravitational Waves from Binary Black Holes by Tuning Higher Harmonics, Phys. Rev. D 98, 084028 (2018).

[84] D. Chiaramello and A. Nagar, Faithful analytical effectiveone-body waveform model for spin-aligned, moderately eccentric, coalescing black hole binaries, Phys. Rev. D 101, 101501 (2020).

[85] A. Nagar, A. Bonino, and P. Rettegno, All in one: Effective one body multipolar waveform model for spin-aligned, quasi-circular, eccentric, hyperbolic black hole binaries, Phys. Rev. D 103, 104021 (2021).

[86] A. Nagar, G. Riemenschneider, G. Pratten, P. Rettegno, and F. Messina, Multipolar effective one body waveform model for spin-aligned black hole binaries, Phys. Rev. D 102, 024077 (2020).

[87] S. Habib and E. Huerta, Characterization of numerical relativity waveforms of eccentric binary black hole mergers, Phys. Rev. D 100, 044016 (2019).

[88] See Supplemental Material at http://link.aps.org/ supplemental/10.1103/PhysRevD.104.024046 for the ancillary files, RRforce.dat.m and modes.dat.m, contain the PN expressions for the RR force and waveform modes for eccentric orbits.

[89] B. R. Iyer and C. Will, PostNewtonian Gravitational Radiation Reaction for Two-Body Systems, Phys. Rev. Lett. 70, 113 (1993).

[90] B. R. Iyer and C. Will, PostNewtonian gravitational radiation reaction for two-body systems: Nonspinning bodies, Phys. Rev. D 52, 6882 (1995).

[91] A. Gopakumar, B. R. Iyer, and S. Iyer, Second postNewtonian gravitational radiation reaction for two-body systems: Nonspinning bodies, Phys. Rev. D 55, 6030 (1997); Erratum, Phys. Rev. D 57, 6562 (1998).

[92] J. Zeng and C. M. Will, Application of energy and angular momentum balance to gravitational radiation reaction for binary systems with spin-orbit coupling, Gen. Relativity Gravity 39, 1661 (2007).

[93] H. Wang and C. M. Will, Post-Newtonian gravitational radiation and equations of motion via direct integration of the relaxed Einstein equations. IV. Radiation reaction for binary systems with spin-spin coupling, Phys. Rev. D 75, 064017 (2007).

[94] A. Buonanno, Y. Chen, and T. Damour, Transition from inspiral to plunge in precessing binaries of spinning black holes, Phys. Rev. D 74, 104005 (2006).
[95] K. Arun, L. Blanchet, B. R. Iyer, and M. S. Qusailah, Inspiralling compact binaries in quasi-elliptical orbits: The complete 3PN energy flux, Phys. Rev. D 77, 064035 (2008).

[96] K. Arun, L. Blanchet, B. R. Iyer, and M. S. Qusailah, Tail effects in the 3PN gravitational wave energy flux of compact binaries in quasi-elliptical orbits, Phys. Rev. D 77, 064034 (2008).

[97] K. Arun, L. Blanchet, B. R. Iyer, and S. Sinha, Third postNewtonian angular momentum flux and the secular evolution of orbital elements for inspiralling compact binaries in quasi-elliptical orbits, Phys. Rev. D 80, 124018 (2009).

[98] N. Loutrel and N. Yunes, Hereditary effects in eccentric compact binary inspirals to third post-Newtonian order, Classical Quant. Grav. 34, 044003 (2017).

[99] L. Blanchet, Gravitational radiation from post-Newtonian sources and inspiralling compact binaries, Living Rev. Relativity 17, 2 (2014).

[100] L. E. Kidder, Coalescing binary systems of compact objects to postNewtonian 5/2 order. 5. Spin effects, Phys. Rev. D 52, 821 (1995).

[101] A. Bohé, S. Marsat, and L. Blanchet, Next-to-next-toleading order spin-orbit effects in the gravitational wave flux and orbital phasing of compact binaries, Classical Quant. Grav. 30, 135009 (2013).

[102] W. G. Dixon, Extended bodies in general relativity: Their description and motion, in Isolated Gravitating Systems in General Relativity, edited by J. Ehlers (North Holland, Amsterdam, 1979), pp. 156-219.

[103] J. Steinhoff, Spin and quadrupole contributions to the motion of astrophysical binaries, Fundam. Theor. Phys. 179, 615 (2015).

[104] W. Tulczyjew, Equations of motion of rotating bodies in general relativity theory, Acta Phys. Pol. 18, 37 (1959); Erratum, 18, 534 (1959).

[105] A. D. Fokker, Relativiteitstheorie (P. Noordhoff, 1929).

[106] M. H. L. Pryce, The mass-centre in the restricted theory of relativity and its connexion with the quantum theory of elementary particles, Proc. R. Soc. Ser. A 195, 62 (1948).

[107] T. D. Newton and E. P. Wigner, Localized states for elementary systems, Rev. Mod. Phys. 21, 400 (1949).

[108] J. Vines, D. Kunst, J. Steinhoff, and T. Hinderer, Canonical Hamiltonian for an extended test body in curved spacetime: To quadratic order in spin, Phys. Rev. D 93, 103008 (2016).

[109] E. Barausse, E. Racine, and A. Buonanno, Hamiltonian of a spinning test-particle in curved spacetime, Phys. Rev. D 80, 104025 (2009); Erratum, Phys. Rev. D 85, 069904 (2012).

[110] N. T. Maia, C. R. Galley, A. K. Leibovich, and R. A. Porto, Radiation reaction for spinning bodies in effective field theory II: Spin-spin effects, Phys. Rev. D 96, 084065 (2017).

[111] A. Bohé, G. Faye, S. Marsat, and E. K. Porter, Quadraticin-spin effects in the orbital dynamics and gravitationalwave energy flux of compact binaries at the 3PN order, Classical Quant. Grav. 32, 195010 (2015).

[112] T. Damour and A. Nagar, Faithful effective-one-body waveforms of small-mass-ratio coalescing black-hole binaries, Phys. Rev. D 76, 064028 (2007). 
[113] T. Mora and C. M. Will, Numerically generated quasiequilibrium orbits of black holes: Circular or eccentric?, Phys. Rev. D 66, 101501 (2002).

[114] A. Gopakumar and B. R. Iyer, Second post-Newtonian gravitational wave polarizations for compact binaries in elliptical orbits, Phys. Rev. D 65, 084011 (2002).

[115] C. K. Mishra, K. Arun, and B. R. Iyer, Third postNewtonian gravitational waveforms for compact binary systems in general orbits: Instantaneous terms, Phys. Rev. D 91, 084040 (2015).

[116] A. Buonanno, G. Faye, and T. Hinderer, Spin effects on gravitational waves from inspiraling compact binaries at second post-Newtonian order, Phys. Rev. D 87, 044009 (2013).

[117] L.E. Kidder, Using full information when computing modes of post-Newtonian waveforms from inspiralling compact binaries in circular orbit, Phys. Rev. D 77, 044016 (2008).

[118] L. Blanchet, G. Faye, B. R. Iyer, and S. Sinha, The Third post-Newtonian gravitational wave polarisations and associated spherical harmonic modes for inspiralling compact binaries in quasi-circular orbits, Classical Quant. Grav. 25, 165003 (2008); Erratum, Classical Quant. Grav. 29, 239501 (2012).

[119] G. Faye, S. Marsat, L. Blanchet, and B. R. Iyer, The third and a half post-Newtonian gravitational wave quadrupole mode for quasi-circular inspiralling compact binaries, Classical Quant. Grav. 29, 175004 (2012).

[120] K. G. Arun, A. Buonanno, G. Faye, and E. Ochsner, Higher-order spin effects in the amplitude and phase of gravitational waveforms emitted by inspiraling compact binaries: Ready-to-use gravitational waveforms, Phys. Rev. D 79, 104023 (2009); Erratum, Phys. Rev. D 84, 049901 (2011).

[121] L. Blanchet, A. Buonanno, and G. Faye, Higher-order spin effects in the dynamics of compact binaries. II. Radiation field, Phys. Rev. D 74, 104034 (2006); Erratum, Phys. Rev. D 75, 049903 (2007); Erratum, Phys. Rev. D 81, 089901 (2010).

[122] Y. Boetzel, C. K. Mishra, G. Faye, A. Gopakumar, and B. R. Iyer, Gravitational-wave amplitudes for compact binaries in eccentric orbits at the third post-Newtonian order: Tail contributions and postadiabatic corrections, Phys. Rev. D 100, 044018 (2019).
[123] S. Drasco, E. E. Flanagan, and S. A. Hughes, Computing inspirals in Kerr in the adiabatic regime. I. The Scalar case, Classical Quant. Grav. 22, S801 (2005).

[124] B. Banihashemi and J. Vines, Gravitomagnetic tidal effects in gravitational waves from neutron star binaries, Phys. Rev. D 101, 064003 (2020).

[125] N. Siemonsen, J. Steinhoff, and J. Vines, Gravitational waves from spinning binary black holes at the leading postNewtonian orders at all orders in spin, Phys. Rev. D 97, 124046 (2018).

[126] T. Damour and A. Nagar, Comparing Effective-One-Body gravitational waveforms to accurate numerical data, Phys. Rev. D 77, 024043 (2008).

[127] T. Damour, B. R. Iyer, and A. Nagar, Improved resummation of post-Newtonian multipolar waveforms from circularized compact binaries, Phys. Rev. D 79, 064004 (2009).

[128] A. Ramos-Buades et al. (to be published).

[129] T. Damour and G. Schäeer, Higher-order relativistic periastron advances and binary pulsars, Il Nuovo Cimento B (1971-1996) 101, 127 (1988).

[130] T. Damour, P. Jaranowski, and G. Schaefer, On the determination of the last stable orbit for circular general relativistic binaries at the third postNewtonian approximation, Phys. Rev. D 62, 084011 (2000).

[131] J. Steinhoff, G. Schaefer, and S. Hergt, ADM canonical formalism for gravitating spinning objects, Phys. Rev. D 77, 104018 (2008).

[132] H. Tagoshi, A. Ohashi, and B. J. Owen, Gravitational field and equations of motion of spinning compact binaries to 2.5 postNewtonian order, Phys. Rev. D 63, 044006 (2001).

[133] C. M. Will, Post-Newtonian gravitational radiation and equations of motion via direct integration of the relaxed Einstein equations. III. Radiation reaction for binary systems with spinning bodies, Phys. Rev. D 71, 084027 (2005).

[134] K. Thorne, Multipole Expansions of Gravitational Radiation, Rev. Mod. Phys. 52, 299 (1980).

[135] G. Cho, B. Pardo, and R. A. Porto, Gravitational radiation from inspiralling compact objects: Spin-spin effects completed at the next-to-leading post-Newtonian order, arXiv:2103.14612.

[136] Y. Pan, A. Buonanno, M. Boyle, L. T. Buchman, L. E. Kidder, H. P. Pfeiffer, and M. A. Scheel, Inspiral-mergerringdown multipolar waveforms of nonspinning blackhole binaries using the effective-one-body formalism, Phys. Rev. D 84, 124052 (2011). 